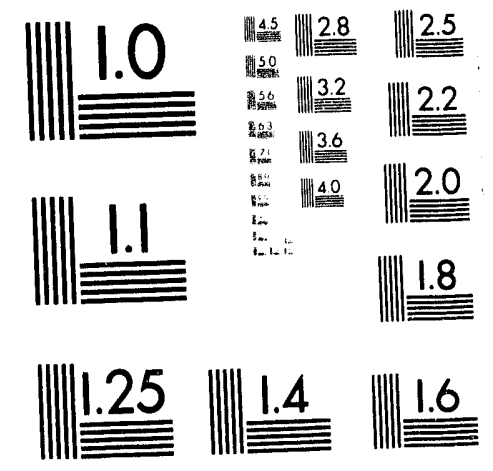



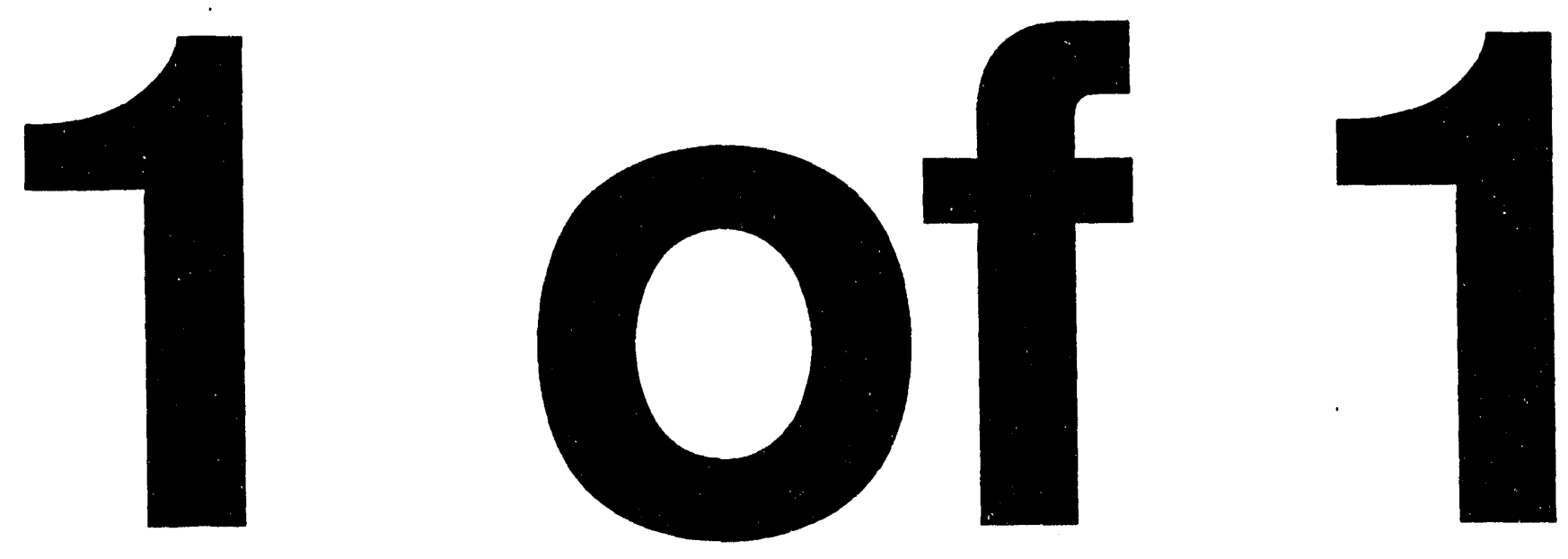
Distribution

SAND93-1603

Category UC-741

Unlimited Distribution

Printed October 1993

\title{
XCHEM-1D \\ A Heat Transfer/Chemical Kinetics Computer Program for Multilayered Reactive Materials
}

\author{
Robert J. Gross \\ Melvin R. Baer \\ Michael L. Hobbs \\ Energetic Materials and Fluid Mechanics Department, 1512 \\ Sandia National Laboratories \\ Albuquerque, New Mexico USA 87185
}

\begin{abstract}
An eXplosive CHEMical kinetics code, XCHEM, has been developed to solve the reactive diffusion equations associated with thermal ignition of energetic materials. This method-of-lines code uses stiff numerical methods and adaptive meshing to resolve relevant combustion physics. Solution accuracy is maintained between multilayered materials consisting of blends of reactive components and/or inert materials. Phase change and variable properties are included in one-dimensional slab, cylindrical and spherical geometries. Temperature-dependent thermal properties have been incorporated and the modification of thermal conductivities to include decomposition effects are estimated using solid/gas volume fractions determined by species fractions. Gas transport properties, including high pressure corrections, have also been included. Time varying temperature, heat flux, convective and thermal radiation boundary conditions, and layer to layer contact resistances have also been implemented.
\end{abstract}
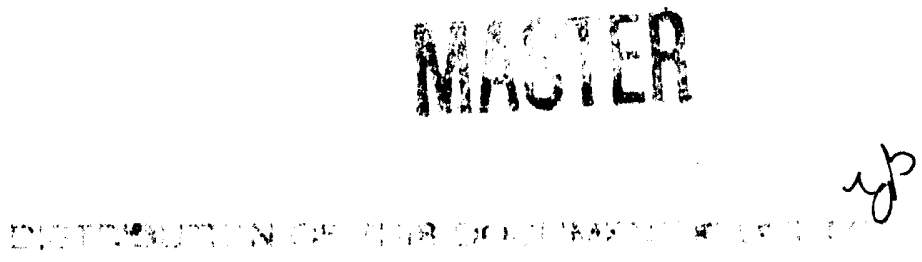


\section{Table of Contents}

PART ONE: MODEL FORMULATION AND SOLUTION

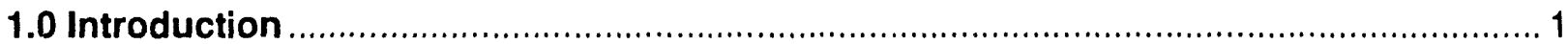

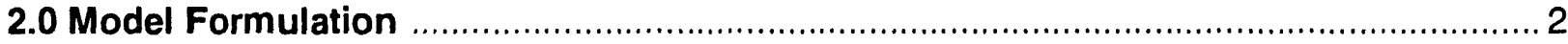

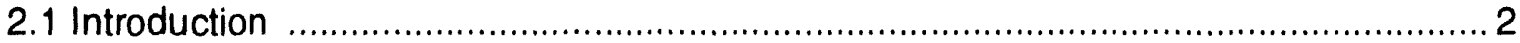

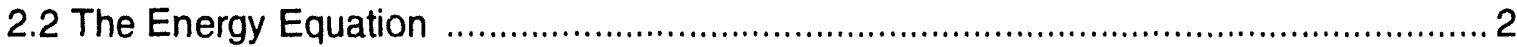

2.3 The Chemical Species Rate Equations ………............................................... 2

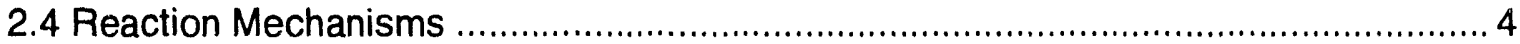

2.5 Reactive Material Properties ............................................................................ 4

2.6 The Effective Capacitance Method ……….....................................................

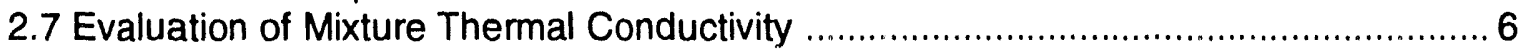

2.8 Evaluation of Mixture Heat Capacity ...................................................................... 8

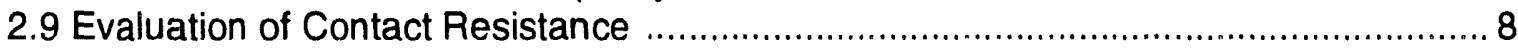

3.0 Numerical Solution Technique ………....................................................................... 10

3.1 Derivations of the Ordinary Differential Equation System ..................................... 10

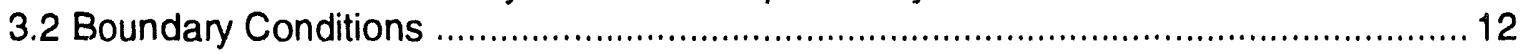

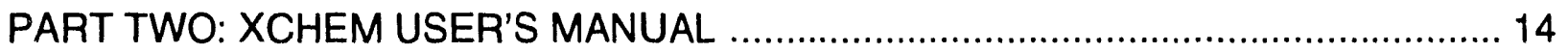

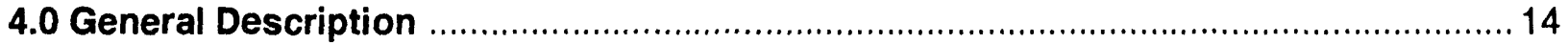

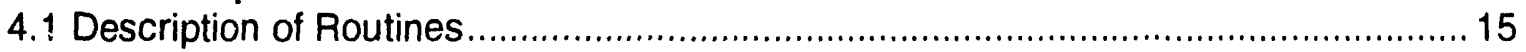

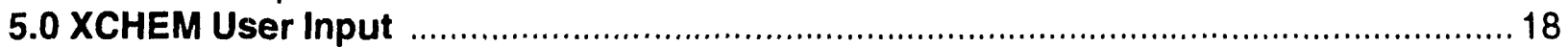

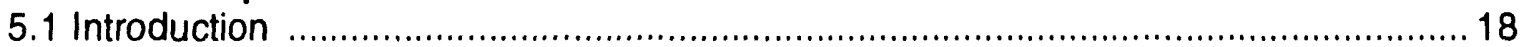

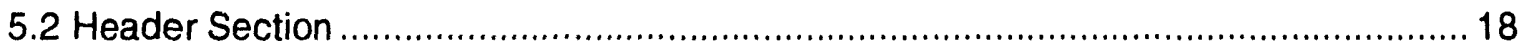

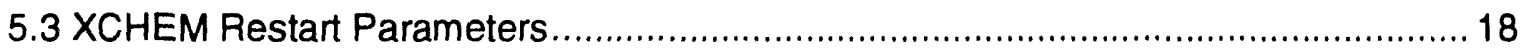

5.4 XCHEM Boundary Condition Input Parameters............................................... 19

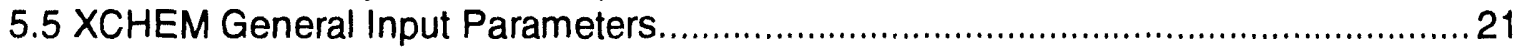

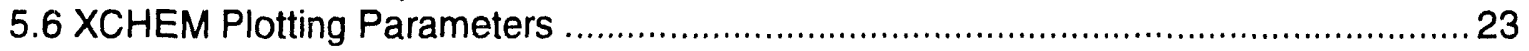

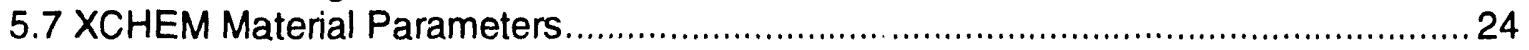

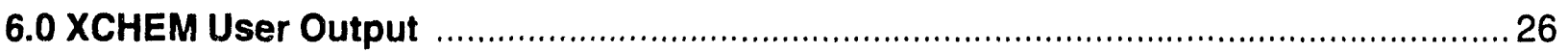

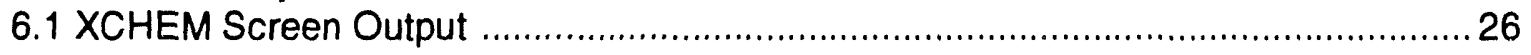

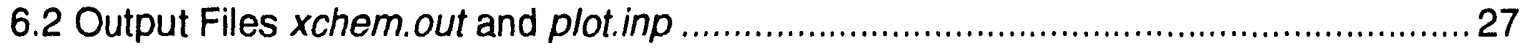

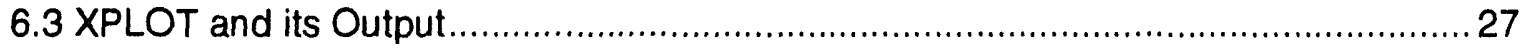

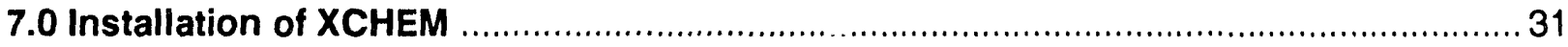

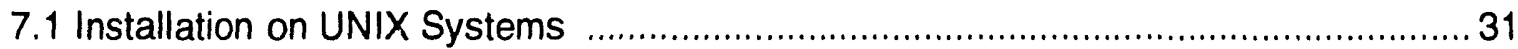

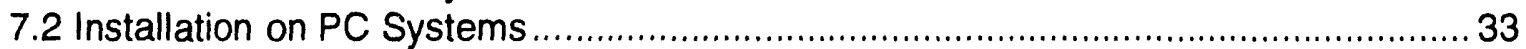

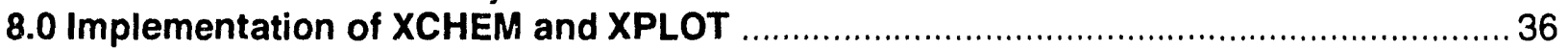

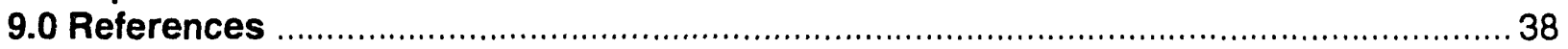

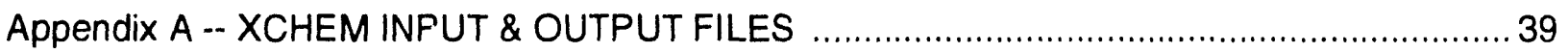

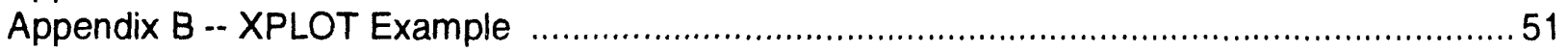

Appendix C -- Derivation of the Concentration Exponent Matrix and the

"Progress Variable" Matrix ............................................................................ 55 


\section{List of Figures}

Figure

Page

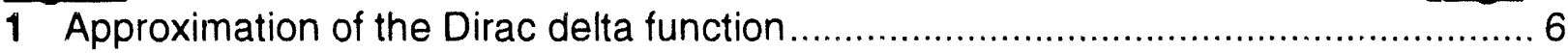

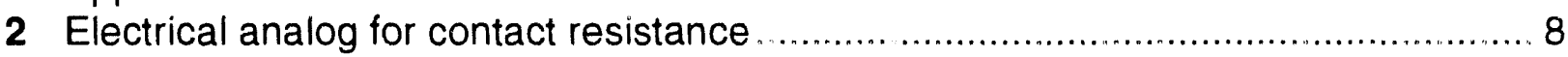

3 Schematic of the dependent variable equation vector.......................................... 11

4 Node and cell designations for XCHEM ........................................................... 12

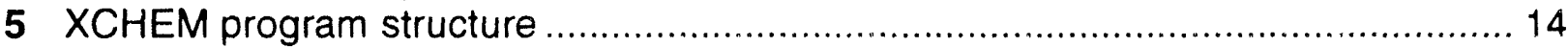

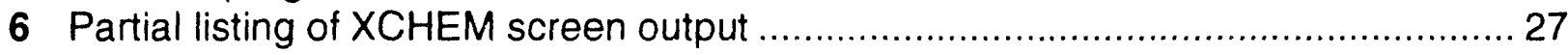

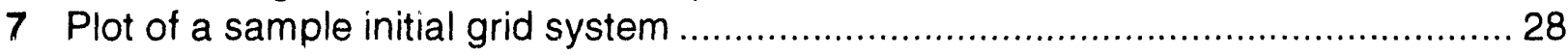

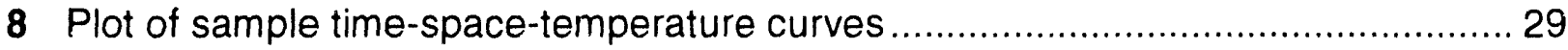

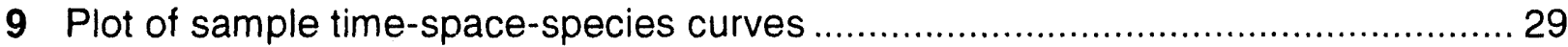

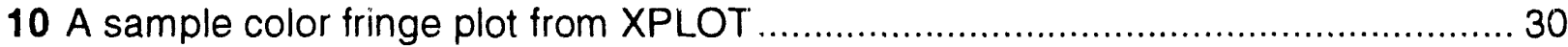

11 A sample three-dimensional time-dependent grid system plot.............................. 30 


\section{PART ONE: MODEL FORMULATION AND SOLUTION}

\subsection{Introduction}

The combined chemical/thermal equations that describe the decomposition of energetic materials, which include explosives and propellants, are complex, highly coupled, and stiff ${ }^{1}$ partial differential equations. Several computer codes ${ }^{2,3}$ have been developed over the last few decades to solve such equation systems, with varying degrees of success. XCHEM-1D, an explosive CHEMical kinetics/heat transfer code was developed because existing codes did not meet all of the following requirements: (1) accurately and robustly solve the coupled stiff chemicalthermal equation system; (2) accurately resolve spatial features such as melt fronts and large species and temperature gradients; (3) allow for different layers where each layer can be composed of different reacting and non-reacting materials in varying proportions; (4) allow flexible and complete boundary condition specification; (5) allow for contact resistance between layers; (6) run with either no or minimal changes on virtually any computing plattorm ranging from a CRAY supercomputer to a PC, and be efficient enough to run reasonably on the slower cpu platforms; (7) be easy to use; (8) be self-contained, including the graphics packages so that external software need not be acquired; (9) provide source for the entire computer code, including the graphics, so that users can make modifications to the code; (10) include a database of properties of common reactive and non-reactive materials and also allow the user to insert "generic" materials into the database through user input and also allow the user to easily add both reacting and non-reacting materials permanently into the code library; (11) allow one node (zero dimensional) calculations which are useful, for example, in probabilistic risk assessment analysis or in deducing chemical kinetics in thin film experiments.

XCHEM-1D (which will interchangeably be called XCHEM) meets all of the above requirements. The method-of-lines (MOL) numerical technique ${ }^{4,5}$ in XCHEM uses stiff numerical solvers to successfully address the problem of obtaining accurate solutions of the temporally stiff chemicalthermal equation system. Automatic adaptive gridding is used to resolve relevant spatial scales. Phase change and variable properties are included in one-dimensional slab, cylindrical and spherical geometries. Temperature-dependent thermal properties have been incorporated. Gas transport properties, including high pressure corrections, have also been included. Time varying temperature, heat flux, convective and thermal radiation boundary conditions, and layer to layer contact resistances have also been implemented. The RSCORS ${ }^{6}$ graphics system is used with XCHEM because source is available for distribution, and it provides striking two- and three-dimensional color graphics. A driver called XPLOT has been written to interface RSCORS to $\mathrm{XCHEM}$ results. This post processor provides quick and easy interpretation of results. XCHEM is easy to use, which is a major achievement for a code that solves such difficult problems. This user friendliness is achieved because the stiff ODE solver (DEBDF) and the adaptive grid routines are extremely robust and have very few parameters that users can modify. Input to XCHEM is typically less than a page, and the majority of input has been arranged such that there is only one data entry per line.

This manual is organized in two parts. Part One discusses the equation system solved by XCHEM, the properties, treatment of phase change, and the MOL solution method. Part Two describes the structure of $X C H E M$, gives a brief description of the core routines, details the input parameters, describes several example problems, and finally discusses the installation of XCHEM on a variety of computing platforms. For those desiring to run XCHEM as soon as possible without a detailed knowledge of the code mechanics or theory, Section 8.1 provides a roadmap to achieve this purpose. 


\subsection{Model Formulation}

\subsection{Introduction}

In the following sections, the formulation of the equations and associated constitutive relations are discussed in more detail. The overall methodology, however, uses the method-of-lines (MOL) to convert the system of partial differential equations (PDE's) describing the decomposition of an energetic material into a system of ordinary differential equations (ODE's). The system of ODE's derived in the following sections are then solved. For each layer, there are a number of spatial gridpoints (similar to a finite-difference approach). Each gridpoint has a temperature, and, optionally (if a reactive material is present), a number of species concentrations that are the unknown dependent variables. The coupled energy equation and the species rate equations are used to solve for these unknowns. The number of species equations may be variable from layer to layer, depending on the number of reacting materials in a particular layer, and the reaction processes associated with each reacting material. Specific heat is determined using the effective capacitance method to allow for the effect of phase change and appropriate mixture rules. The logarithm of thermal conductivity is assumed to vary linearly with temperature. The reacting materials available in the material library consist of $\mathrm{CHNOCl}$ atoms and decompose to a representative gas composition consisting of $\mathrm{N}_{2}, \mathrm{H}_{2}, \mathrm{H}_{2} \mathrm{O}, \mathrm{O}_{2}, \mathrm{CO}_{2}, \mathrm{CO}, \mathrm{HCl}$, and $\mathrm{Cl}$. Since the composition is assumed constant, computation of gas properties, including density and thermal conductivity can be computed using the pure substance values and high pressure corrections to well-known mixture rules. Further, arbitrary generic reactive and nonreactive materials can easily be added to the XCHEM library via user input.

\subsection{The Energy Equation}

The temperature field in a heated energetic material is described in XCHEM by the following equation for a multilayer, multicomponent reacting system:

$$
\rho C_{\mathrm{P}} \frac{\partial T}{\partial t}=\lambda \nabla^{2} T+S_{T}
$$

where $\rho\left(\mathrm{g} / \mathrm{cm}^{3}\right), C_{\mathrm{p}}(\mathrm{cal} / \mathrm{g}-\mathrm{K}), T(\mathrm{~K}), t(\mathrm{~s}), \lambda(\mathrm{cal} / \mathrm{cm}-\mathrm{s}-\mathrm{K})$, and $S_{T}\left(\mathrm{cal} / \mathrm{cm}^{3} \mathrm{~s}\right)$ represent density, temperature dependent heat capacity, temperature, time, temperature dependent thermal conductivity, and reaction source term, respectively. The thermophysical quantities are computed using mixture rules and are discussed in Sections (2.6) to (2.8). The total mixture density is constant in Equation (1) since no material expansion or depletion in the confinement is considered in this version of XCHEM, thus the volume and mass of the system is fixed. For one-dimensional geometries, the Laplacian operator, $\nabla^{2}$, has the form $\partial^{2} / \partial x+(m / x) \partial / \partial x$, where $m$ is 0,1 , or 2 , for cartesian or slab, cylindrical, and spherical geometries, respectively. Combined radiative and convective boundary conditions, including Dirichlet (specified time varying temperature) or Neumann (specified variable flux) conditions, have been incorporated into XCHEM. Implementation of these boundary conditions is discussed in detail in Section (3.2).

\subsection{The Chemical Species Rate Equations}

The volumetric reaction source term, $S_{T}\left(\mathrm{cal} / \mathrm{cm}^{3} \mathrm{~s}\right)$, given in Equation (1) above is determined by the following source term for $\mathrm{J}$ reactions involving $\mathrm{K}$ materials:

$$
S_{T}=\sum_{k=1}^{K} \sum_{j=1}^{J} \mathscr{H}_{k j} \rho_{k} r_{k j}
$$


where $\mathcal{H}_{k j}(\mathrm{caV} / \mathrm{g}), \rho_{k}\left(\mathrm{~g} / \mathrm{cm}^{3}\right)$, and $r_{k j}(1 / \mathrm{s})$ represent the endothermic or exothermic energy release, the density, and the reaction rate of the $k^{\text {th }}$ material for reaction step $\mathrm{j}$, respectively. The general reaction scheme for $\mathrm{J}$ reactions involving I chemical species is:

$$
\sum_{i=1}^{1} v^{\prime}{ }_{i j} \mathcal{M}_{i} \rightarrow \sum_{i=1}^{1} v^{\prime \prime}{ }_{i j} \mathcal{M}_{i}, \quad j=1, \ldots J
$$

where $v_{i j}^{\prime}, v_{i j}^{\prime \prime}$ and $M_{i}$ are the stoichiometric coefficients of the reactants and products, and the chemical symbol for the $i$ th species, respectively. Although the reactions in Equation (3) can be reversible, the reverse reactions are specified in XCHEM as additional reaction steps. Equation (3) in general describes elementary chemical kinetics in which $v_{i j}^{\prime}$ and $v^{\prime \prime}{ }_{i j}$ usually must be integer values. In addition, most global reaction schemes follow the premise of having $J$ reactions involving I chemical species and such a conceptual picture is useful for the remaining formulation. However, Equation (3) cannot always be applied to the global kinetics used to describe the chemistry of some energetic systems. For example, in some global kinetic models, the stiochiometric coefficients need not be integer values (i.e. reaction rates can be specified as a complex, nonlinear function of the reactants and products). As the ensuing discussion will clarify, $\mathrm{XCHEM}$ allows both global or elementary reaction mechanisms.

For each of the $\mathrm{j}$ reaction steps, the reaction rate, $r_{j}(1 / s)$ is described by:

$$
r_{j}=k_{j} \prod_{i=1}^{\prime} N_{i}^{\mu \prime}, \quad j=1, \ldots J
$$

where $N_{i}$ (dimensionless) is a progress variable that tracks the creation or destruction of species $i$ and the $\mu_{i j}$ (dimensionless) are termed the concentration exponents. Equation (4) as it appears here is very general and encompasses both elementary and global kinetics models. In elementary kinetics, $\mu_{i j}=v^{\prime}{ }_{i j}$, but this is not the case for most global kinetic models that are typically used in XCHEM. Thus, for generality, we have chosen not to relate the stoichiometric coefficient to the concentration exponent. Also, in the form of Equation (4), $N_{i}$ can be a concentration of species $i$, a mole fraction, or a mass fraction, provided that appropriate reaction rates, $r_{j}$, and the kinetic coefficients, $k_{j}$, are defined consistently. Several examples for consistent definitions are shown in Table 2. The expressions for the kinetic coefficients, $k_{j}(1 / \mathrm{s})$, are given in an Arrhenius form:

$$
k_{j}=T^{\beta_{j}} A_{j} \exp \left(-E_{j} / R T\right)
$$

where $\beta_{j}, A_{j}\left(1 / \mathrm{K}^{\beta} \mathrm{s}\right), E_{j}(\mathrm{cal} / \mathrm{mol})$, and $R(1.987 \mathrm{cal} / \mathrm{mol}-\mathrm{K})$ are the steric coefficients, pre-exponential factors, activation energies, and the universal gas constant, respectively. The rates of change of species are given by:

$$
d N_{i} / d t=\sum_{J=1}^{J} v_{i j} r_{j}, \quad i=1, \ldots l
$$

where $v_{i j}$ (dimensionless) are the coefficients on each of the $j$ reaction rates. Appendix $C$ presents examples of global kinetics models where the two matrices $\mu_{i j}$ and $v_{i j}$ are derived. Equations (1) and (6) form an equation system that, together with auxiliary Equations (2)-(5), can be used to determine the temperature and species history leading to ignition providing the decomposition mechanisms, temperature and pressure dependent properties are known. As discussed above, Equation (3) need not be invoked; indeed, none of the variables in Equation (3) appear in Equations (4)-(6). 


\subsection{Reaction Mechanisms}

A major problem with incorporating chemical kinetics for condensed phase materials is the lack of mechanistic rate data. Experimental methods for obtaining this information are not well established. As discussed in the previous section, most kinetic models for condensed phase materials are global, and usually consist of only a few steps. The multistep kinetics model with parameters which replicate the time to reaction as observed from the heavily confined One-Dimensional Time to Explosion (ODTX) experiments of McGuire and Tarver ${ }^{7}$ have been incorporated into XCHEM. Table 1 lists the mechanisms for HMX. RDX. TATB, TNT, and NC. The mechanism for HMX and RDX is known as a three-step sequential mechanism and the mechanism for TATB and TNT is known as a three-step auto-catalytic mechanism. See Appendix $\mathrm{C}$ for additional details on these mechanisms. Generic mechanisms for an arbitrary reactive material are allowed; presently, in XCHEM the generic mechanism is limited to nineteen steps.

Table 1. Reaction mechanisms for primary explosives.

\begin{tabular}{|c|c|c|}
\hline HMX and RDX & TA'TB and TNT & $\mathrm{NC}$ \\
\hline$A \rightarrow B$ & $A \rightarrow B$ & $1 \quad A \rightarrow B$ \\
\hline$B \rightarrow 2 C$ & $A+B \rightarrow C$ & $2 \quad A+B \rightarrow C$ \\
\hline $2 C \rightarrow D$ & $B+B \rightarrow C$ & \\
\hline
\end{tabular}

\subsection{Reactive Material Properties}

The Arrhenius kinetic parameters for the mechanisms given in Table 1, including reaction energies, are listed in Table 2. The steric coefficients, $\beta$, for these explosives are all zero. The temperature dependent specific heats and thermal conductivities used in XCHEM are also included in Table 2.

Table 2. Kinetic and thermal properties used in XCHEM

\begin{tabular}{|l|l|l|l|l|l|}
\hline Explosive & HMX & RDX & TATB & TNT & NC \\
\hline$y_{1}$, zaVg & -100 & -100 & -50 & -30 & -30 \\
\hline $\mathrm{Ln}^{\dagger} A_{1}, \mathrm{Ln}(1 / \mathrm{s})$ & 48.7 & 45.5 & 29.5 & 35.0 & 35.6 \\
\hline$E_{1} / R, \mathrm{~K}$ & 26500 & 23700 & 21100 & 22100 & 19600 \\
\hline $\mathscr{H}_{2}, \mathrm{caV} / \mathrm{g}$ & 300 & 300 & 900 & 900 & 630 \\
\hline $\mathrm{Ln} A_{2}, \mathrm{Ln}(1 / \mathrm{s})$ & 37.5 & 40.7 & 45.0 & 26.0 & 32.0 \\
\hline$E_{2} / R, \mathrm{~K}$ & 22200 & 22200 & 30200 & 17400 & 13200 \\
\hline $\mathcal{H}_{3}, \mathrm{caV} / \mathrm{g}$ & 1200 & 1200 & 950 & 930 & $\mathrm{NA}$ \\
\hline $\mathrm{Ln} A_{3}, \mathrm{Ln}(1 / \mathrm{s})$ & 28.1 & 35.0 & 43.5 & 26.2 & $\mathrm{NA}$ \\
\hline$E_{3} / R_{1} \mathrm{~K}$ & 17200 & 17200 & 27200 & 16900 & $\mathrm{NA}$ \\
\hline$C_{p}, \mathrm{cal} / \mathrm{g}-\mathrm{K}, 293 \mathrm{~K}$ & 0.24 & 0.24 & 0.26 & 0.27 & 0.24 \\
\hline
\end{tabular}




\begin{tabular}{|l|l|l|l|l|l|}
\hline Explosive & HMX & RDX & TATB & TNT & NC \\
\hline$c_{p}, \mathrm{cal} / \mathrm{g}-\mathrm{K}, 623 \mathrm{~K}$ & 0.42 & 0.42 & 0.43 & 0.40 & 0.42 \\
\hline$\lambda, \mathrm{calcm}-\mathrm{s}-\mathrm{K}, 293 \mathrm{~K}$ & 0.00123 & 0.000622 & 0.00191 & 0.000620 & 0.00123 \\
\hline$\lambda, \mathrm{cal} / \mathrm{cm}-\mathrm{s}-\mathrm{K}, 433 \mathrm{~K}$ & 0.000970 & 0.000485 & 0.00142 & 0.000570 & 0.000970 \\
\hline$\rho, \mathrm{g} / \mathrm{cm}^{3}$ & 1.9 & 1.8 & 1.842 & 1.605 & 1.65 \\
\hline Melting Point, $\mathrm{K}$ & 520 & 477 & NA & 354 & $\mathrm{NA}$ \\
\hline Latent Energy, cal/g & 50.0 & 38.4 & NA & 22.3 & NA \\
\hline
\end{tabular}

*Most parameters are from references 1 and 2.

tLn represent the natural logarithm.

\subsection{The Effective Capacitance Method}

Some energetic materials such as TNT melt before ignition. Since the melting process includes a latent heat, it is important to include this phenomenon in the energy equation. However, the time and location of the phase change is not known a priori, occurs in a small region, usually moves, and continuity of the temperature field is required. For spatial resolution, the adaptive grid technique accurately resolves and tracks the phase change front. To account for the latent energy and to assure continuity of temperature, the effective capacitance model ${ }^{8}$ is used.

Equation (1) is again given as:

$$
\rho C_{p}^{\star} \frac{\partial T}{\partial t}=\lambda \nabla^{2} T+S_{T}
$$

where $C_{p}{ }^{*}$ is now the equivalent specific heat defined by:

$$
C_{P}^{*}=\frac{d H}{d T}=C_{P}(T)+\mathcal{L} \delta\left(T-T_{m}\right)
$$

where $\mathrm{H}\left(\mathrm{cal} / \mathrm{cm}^{3} \mathrm{~g}\right)$ is the enthalpy, $\mathcal{L}$ (cal) is the latent energy, $\delta$ is the Dirac delta function, and $T_{m}(\mathrm{~K})$ is the phase transition or melt temperature. This is a computationally effective approach ${ }^{9}$ because a two phase region with a jump condition has been reduced to a single phase region with rapidly varying properties. In XCHEM, Equation (8) is replaced by:

$$
C_{p}{ }^{*}=C_{p}(T)+\mathcal{L} \delta^{*}\left(T-T_{m}, \Delta T\right)
$$

where $\delta$ is the delta form function. The form function has a finite value in the interval $\Delta T$ about $T_{m}$ and is zero outside the interval. In XCHEM, the approximation user for of the delta function is shown in Figure 1 and by definition the area under the curve is equal to $\int_{-\infty}^{\infty} \delta d T=1$ such that:

$$
\delta^{*}\left(T-T_{m} \Delta T\right)=\frac{1}{\varepsilon^{2}} \max \left(0.0, \varepsilon-\mid T-T_{m}\right)
$$

The value of $\varepsilon$ is the temperature range over which phase change occurs and is arbitrarily set to $1 \mathrm{~K}$. 


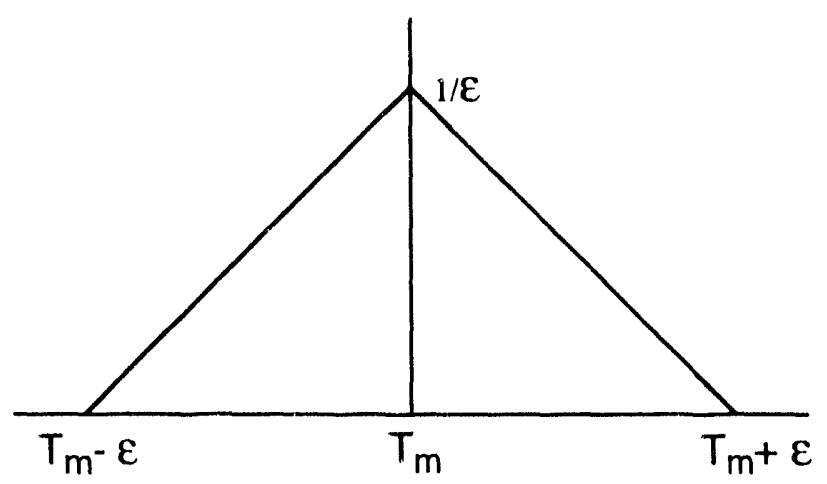

Figure 1. Approximation of the Dirac delta function

\subsection{Evaluation of Mixture Thermal Conductivity}

The value of thermal conductivity can vary greatly in a mixture depending on the state (solid or gas) of the components in that mixture. The solid thermal conductivities are evaluated by interpolating on tabular values provided in the XCHEM database. It is assumed that the logarithm of thermal conductivity is linear with respect to temperature. Therefore, the natural logarithm of tabular values are taken, a linear interpolation is made, and the value is reconverted to normal space by taking the exponential. The solid mixture thermal conductivity is evaluated from:

$$
\lambda_{\text {solid }}=\sum_{i=1}^{n \max } \chi_{i} \lambda_{i}
$$

where $\chi_{i}$ is the volume fraction of the solid material in the layer, and $\lambda_{i}(\mathrm{caV} / \mathrm{cm}-\mathrm{s}-\mathrm{K}$ ) is the pure material temperature-dependent thermal conductivity. The summation is made over nmax, the number of solid materials in the layer.

Since the composition of the decomposition gases in a layer are assumed constant in XCHEM, a mixture gas thermal conductivity is calculated. However, XCHEM makes a high pressure correction to this initial value that varies with time. First, for each pure gas, the thermal conductivity is calculated ${ }^{10}$ :

$$
\lambda_{\text {puregas }}=\frac{1.989 \times 10^{-4}(T / M)^{1 / 2}}{\sigma^{2} \Omega_{v}}
$$

where $T(K)$ is the temperature, $M$ is the molecular weight, $\sigma(\AA)$ is a collision diameter, and $\Omega_{v}$ (dimensionless) is the collision integral. The collision integral is approximated ${ }^{11}$ as:

$$
\Omega_{v}=\left(\frac{A}{T^{*} B}\right)+\frac{C}{\exp \left(D T^{*}\right)}+\frac{E}{\exp \left(F T^{*}\right)}
$$

where $T^{\prime}=(k T) / \varepsilon, A=1.16145, B=0.14874, C=0.52487, D=0.77320, E=2.16178$, and $F=2.43787 . \quad T$ is a dimensionless temperature related to the dimensional temperature by $k$, the Boltzmann constant, and the characteristic energy, $\varepsilon$, which is usually given as a single value, $\varepsilon / k$ and has dimensions of $1 / K$. Values for $\varepsilon / k$ and $\sigma$ for many substances may be found in reference (12). The mixture gaseous thermal 
conductivity is found using the Wassiljewa equation:13

$$
\lambda_{\mathrm{m}}^{o}=\sum_{i=1}^{n} \frac{y_{i} \lambda_{1}}{\left(\sum_{j=1}^{n} \lambda_{j} A_{i j}\right)}
$$

where $\lambda_{m}^{o}$ is the low pressure thermal conductivity of the mixture, $\lambda_{i}$ is the thermal conductivity of the pure substance, and $y_{1}$, are the mole fractions of components $\mathrm{i}$. The function $A_{11}$ is computed by using the Mason and Saxena modification ${ }^{13}$ :

$$
A_{i j}=\frac{\left[1+\left(\eta_{i} M_{j} / \eta_{j} M_{j}\right)^{1 / 2}\left(M_{i} / M_{j}\right)^{1 / 4}\right]^{2}}{\left[8\left(1+M_{i} / M_{j}\right)\right]^{1 / 2}}
$$

where $\eta$ (micropoise) is the viscosity of the pure substance and all other quantities have been previously defined. The pure gas viscosity is computed from.

$$
\eta=\frac{\sqrt{M T}}{\sigma^{2} \Omega_{v}}
$$

Equations (11) to (16) determine the low pressure mixture thermal conductivity for the known gas mixture. This value is only calculated once, and is performed in XCHEM before it begins to step in time. The final high pressure correction technique of Stiel and Thodos ${ }^{14}$, is computed on every timestep for the energy equation is:

$$
\begin{array}{cc}
\lambda=\lambda_{m}^{0}+\left(14.0 \times 10^{-8}\right)\left(e^{0.535 \rho_{r}}-1\right) / \Gamma & \rho_{r}<0.5 \\
\lambda=\lambda_{m}^{0}+\left(13.1 \times 10^{-8}\right)\left(e^{0.67 \rho_{r}}-1.069\right) / \Gamma & 0.5<\rho_{r}<2.0 \\
\lambda=\lambda_{m}^{o}+\left(2.976 \times 10^{-8}\right)\left(e^{1.155 \rho_{r}}+2.016\right) / \Gamma & 2.0<\rho_{r}<2.8
\end{array}
$$

where $\rho$, is the reduced density, $\rho_{r}=\rho / \rho_{c}, \lambda_{m}^{0}$ is the low pressure thermal conductivity, and

$$
\Gamma=\frac{T_{c}^{1 / 6} Z_{c}^{5} M^{1 / 2}}{P_{c}^{2 / 3}}
$$

All quantities witt. a subscript "c" are critical values. $Z_{c}$ is the compressibility factor for the substance. For a particular substance, $\Gamma$, is a constant value and need be computed only once. The mixture critical thermodynamic properties, $\zeta=P, T$; 'etc. are computed from: 


$$
\zeta_{\mathrm{m}}^{\mathrm{c}}=\sum_{i=1}^{n} \frac{y_{i} \zeta_{i}^{\mathrm{c}}}{\left(\sum_{j=1}^{n} y_{j} \zeta_{j}^{\mathrm{c}}\right)}
$$

Equations (17a)-(17c) and (18) can be efficiently jrogrammed into a function subroutine. The solid mixture thermal conductivity and gas mixture thermal conductivity are density-weighted to obtain the final thermal conductivity at each gridpoint:

$$
\lambda=\lambda_{\text {solid }}\left(1.0-\frac{\rho_{\text {gas }}}{\rho_{\text {solid }}}\right)+\lambda_{\text {gas }} \frac{\rho_{\text {gas }}}{\rho_{\text {solid }}}
$$

\subsection{Evaluation of Mixture Heat Capacity}

Only the initial materials in a layer are used to evaluate the heat capacity of the mixture. For each material (both reacting and nonreacting), the heat capacity is linearly interpolated using the XCHEM heat capacity database. Then, the density/specific heat product is determined from:

$$
\rho C_{p}=\sum_{i=1}^{n \max } \chi_{i} \rho_{i} C_{p_{i}}
$$

where $\chi_{i}, \rho_{i}\left(\mathrm{~g} / \mathrm{cm}^{3}\right)$, and $c_{\rho_{i}}(\mathrm{caV} / \mathrm{g}-\mathrm{K})$ are the initial volume fraction of the material, the species density, and the species specific heat, respectively. The specific heat of the gaseous product materials are assumed to be similar to the solid specific heats in these confined geometries.

\subsection{Evaluation of Contact Resistance}

The evaluation of contact resistance involves computing an effective thermal conductivity modified from the value in Section 2.7. Contac resistance is only allowed presently at layer interfaces. Contact resistance is computed from standard heat transfer principles and the electrical analogy to heat flow. Both radiation and conduction through the gap are included. Figure 2 depicts the assumed electrical analogy network for the contact resistarice. The radiation heat flux is ${ }^{15}$ :

$$
(q / A)_{\text {radiation }}=h_{\text {rad }}\left(T_{1}-T_{2}\right)
$$

where $q\left(\mathrm{cal} / \mathrm{s} \mathrm{cm}^{2}\right)$ is the heat transfer rate, $A\left(\mathrm{~cm}^{2}\right)$ is the area, and $\mathrm{h}_{\mathrm{rad}}\left(\mathrm{cal} / \mathrm{s} \mathrm{cm^{2 }} \mathrm{K}\right)$ is the radiation

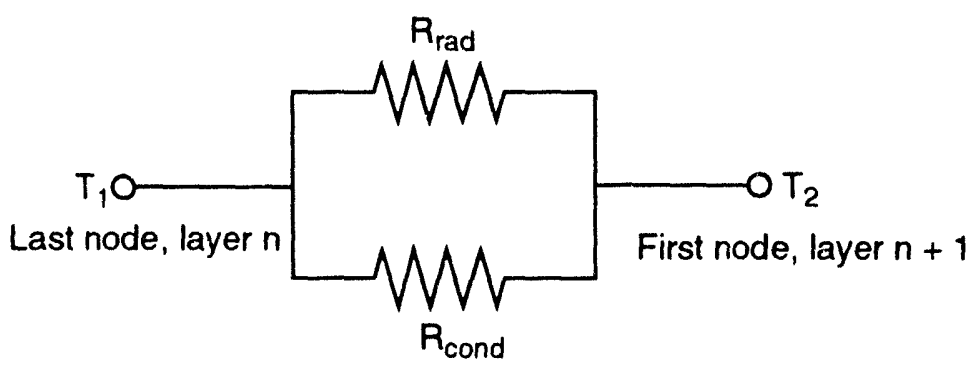

Figure 2. Electrical analog for contact resistance. 
coefficient defined by:

$$
h_{\text {rad }}=\frac{\sigma\left(T_{1}^{2}+T_{2}^{2}\right)\left(T_{1}+T_{2}\right)}{L_{g}\left[1 / \varepsilon_{1}+\left(A_{1} / A_{2}\right)\left(1 / \varepsilon_{2}-1\right)\right]}
$$

where $\sigma\left(\mathrm{cal} / \mathrm{s} \mathrm{cm}^{2} \mathrm{~K}^{4}\right)$ is the Stefan-Boltzmann radiation constant, $\mathrm{L}_{\mathrm{g}}(\mathrm{cm})$ is the gap width, and the $\varepsilon^{\prime} \mathrm{s}$ (dimensionless) are the gray body emissivities of the two gap surfaces. In XCHEM, $A_{1}=A_{2}$, so that these terms cancel and do not appear. Likewise, for the conduction heat flux ${ }^{16}$ we have:

$$
(q / A)_{\text {conduction }}=h_{\text {cond }}\left(T_{1}-T_{2}\right)
$$

where $h_{\text {cond }}\left(\mathrm{cal} / \mathrm{s} \mathrm{cm}^{2} \mathrm{~K}\right)$ is the conduction coefficient defined as:

$$
\mathrm{h}_{\text {cond }}=\frac{1}{L_{\mathrm{g}}}\left(\frac{A_{\mathrm{c}}}{A} \frac{2 \lambda_{\mathrm{A}} \lambda_{\mathrm{B}}}{\lambda_{\mathrm{A}}+\lambda_{\mathrm{B}}}+\frac{A_{\mathrm{v}}}{A} \lambda_{\mathrm{f}}\right)
$$

where $A_{c}\left(\mathrm{~cm}^{2}\right)$ is the contact area, $A_{v}\left(\mathrm{~cm}^{2}\right)$ is the void area, $A\left(\mathrm{~cm}^{2}\right)$ is the total area, the subscripts "A" and " $\mathrm{B}$ " denote the different materials on either side of the gap, the subscript " $"$ " denotes the fluid material which fills the void space, and $\lambda$ (cals $\mathrm{cm} \mathrm{K}$ ) is thermal conductivity.

In XCHEM, each node is assigned a thermal conductivity in which the thermal resistance is:

$$
R_{\text {normal }}=\frac{1}{\lambda}
$$

where $\lambda$ is obtained from Equation (20). The contact resistance is implemented by replacing the normal thermal resistance with the effective contact resistance:

$$
R_{\text {contact }}=\frac{\left(1 / h_{\text {cond }}\right)\left(1 / h_{\mathrm{rad}}\right)}{\left(1 / h_{\text {cond }}\right)+\left(1 / h_{\text {rad }}\right)}
$$

and $\mathrm{R}_{\text {contact }}$ in Equation (27) is used to define an effective "thermal conductivity" instead of Equation (26). Mechanically-induced contact resistance is not implemented in the present version of XCHEM. 


\subsection{Numerical Solution Technique}

\subsection{Derivation of the Ordinary Differential Equation System}

For each node in the grid system, the energy equation (1) and a number of species rate change equations (6) are solved. The method of lines (MOL) numerical method is well-suited to solving such an equation system, especially one in which there is grid adaption and the number of equations solved is constantly evolving. The first step in MOL is to discretize the spatially dependent terms to create a system of coupled, first-order ordinary differential equations (ODE's) in time. At present, since species diffusion is not included, only the energy diffusion term of equation (1) has a spatially dependent term. If we define

$$
q_{k+\frac{1}{2}} \equiv \frac{\left(T_{k+1 / 2}-T_{k}\right)}{\left(x_{k+1}-x_{k}\right) / 2}
$$

where $q(\mathrm{~K} / \mathrm{cm})$ is the temperature gradient and $x(\mathrm{~cm})$ is the spatial location. Further, in considering variable thermal conductivity, a simple matching of heat fluxes in the positive and negative directions (in one dimension) at a node results in the following definition:

$$
T_{k+\frac{1}{2}} \equiv \frac{\left(\lambda_{k} T_{k}+\lambda_{k+1} T_{k+1}\right)}{\left(\lambda_{k+1}+\lambda_{k}\right)}
$$

The spatial operator in equation (1) becomes:

$$
\nabla^{2} T \propto\left(A_{k+\frac{1}{2}} q_{k+\frac{1}{2}}-A_{k-\frac{1}{2}} q_{k-\frac{1}{2}}\right), V_{k}
$$

where $V$ is the volume of the computational cell surrounding a gridpoint and $A_{k+\frac{1}{2}}$ is the interface area between $k$ and $k+1$ adjacent cells. Variable areas and volumes are defined for cylindrical and spherical geometries. Once the spatial operator is discretized, the equation system at gridpoint $k$ consists of:

$$
\begin{gathered}
\frac{d T_{k}}{d t}=\left(\frac{\lambda}{\rho C_{p}}\right)\left[\left(\left(\frac{2 \lambda_{k+1}}{\lambda_{k+1}+\lambda_{k}}\right) A_{k+\frac{1}{2}}\left(T_{k+1}-T_{k}\right)-\left(\frac{2 \lambda_{k-1}}{\lambda_{k-1}+\lambda_{k}}\right) A_{k+\frac{1}{2}}\left(T_{k}-T_{k-1}\right)\right) / V_{k}\right]+\left(\frac{1}{\rho C_{p}}\right){ }_{k}\left(\sum_{j=1}^{J} \mathcal{H}_{j} \rho r_{j}\right) \\
\frac{d N_{t}}{d t}=\sum_{j=1}^{J} v_{i j} T^{\beta /} A_{j} \exp \left(-E_{j} / R T \prod_{i=1}^{1} N_{i}^{\mu_{i}}\right.
\end{gathered}
$$

Each gridpoint has a system composed of equations (31)-(32) associated with it. The unknown vector $f$ passed to the MOL solver is shown in Figure 3. (This is the name of the vector in XCHEM). In Figure 3 , $n p t$ refers to the total number of nodes, or gridpoints, in the computational domain at the current time. As a clarification, the words gridpoint and node are used interchangeably to indicate a discrete computational point. With adaptive gridding, this value changes with time. The reference to npde refers to the number of partial differential equations associated with a node. Note that the value of npde can vary from layer to layer, e.g. if Node 1 and Node npt are in different layers, the value of npde as a subscript of their respective unknown f's may differ. To further clarify the form of the $f$ array in Figure 3 , for each node, its $f$ component 


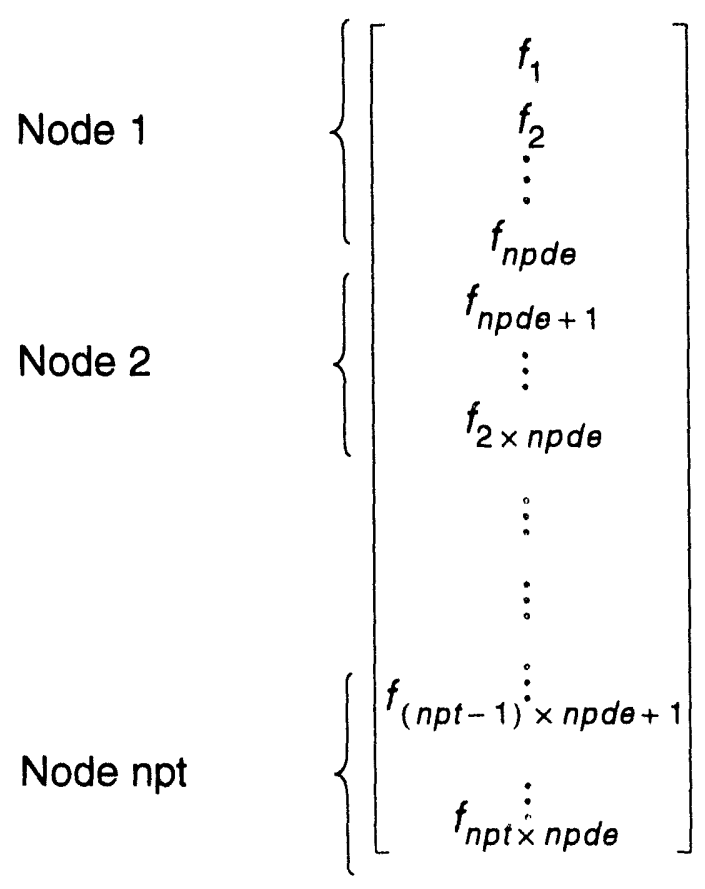

Figure 3. Schematic of the dependent variable equation vector

consists of:

$$
\mathbf{f}_{\text {node } T}=\left(T, N_{A}, N_{B}, \ldots, N_{1}\right)^{T}
$$

where $I=$ npde -1 and the superscript $\mathcal{T}$ represents the vector transpose. Each node has npde unknowns, which includes the temperature. The remaining unknowns are species, and thus there must be npde-1 unknown species.

The concentration exponent matrix, $\mu_{i j}$, and the coefficient matrix, $v_{i j}$, must be known in order to solve Equation (32). Appendix $C$ provides a derivation of $\mu_{i j}$ and $v_{i j}$ for a sequential three-step mechanism, and an autocatylitic three-step mechanism. The former is used in XCHEM to describe the chemical decomposition mechanism for HMX and RDX, while the latter is used to describe the mechanism for TATB and TNT. Using Appendix $C$ as a guideline, these necessary matrices can be obtained for a generic mechanism (up to nineteen steps) that can be entered into XCHEM as input.

The equation system (31-32) is solved using the double precision version of an ODE solver called DEB$D^{17}$, which is a variable order backward difference solver. This ODE method forms a Jacobian, which can require substantial storage. To minimize storage, the bandwidth of the Jacobian has been minimized by determining the layer with the most unknown reactant species and using this information to compute the bandwidth. Early versions XCHEM used a bandwidth that addressed the possibility of all the reactant materials in the XCHEM library being present in all layers. Reducing the bandwidth reduced XCHEM cpu time by an order of magnitude. 
The adaptive gridding is performed by layer, as opposed to the solver which advances the solution of the entire computational domain. Performing the adaptive gridding by layer eliminates grid smoothing difficulties. Relative error estimates are computed for each dependent variable on each gridpoint node by using error estimates based on interpolation. A cumulative error estimate for each node is also obtained by calculatinging a weighted sum of the component errors for individual nodes. A node is a candidate for refinement (node insertion) if the error exceeds a preset tolerance, $\sigma_{0}$. A node is a candidate for combination with a neighboring node (node removal) if the error is less than another specified tolerance (for instance, $0.1 \sigma_{0}$ ). An automatic smoothness restriction is also imposed so that node widths cannot be more than twice as large or less than half as small as its nearest node neighbors. ${ }^{18}$

\subsection{Boundary Conditions}

General boundary conditions are incorporated in XCHEM. Figure 4 depicts the node and cell arrangement of XCHEM. The boundary on the left-hand side where node 0 is located is called the "inner boundary" The boundary on the right-hand side where node npt +1 is located is called the "outer boundary". Presently, only boundary conditions for the energy equation are necessary since species diffusion is neglected. Equation (31) in Section 3.1 is the energy equation used for interior nodes. In XCHEM, the right-hand side (RHS) of the energy equation at the boundaries are:

$$
\begin{aligned}
& R H S_{\text {node } 1}=\left(\frac{\lambda}{\rho C_{\rho}}\right),\left(\left(\left(\frac{2 \lambda_{1}}{\lambda+\lambda_{2}}\right) A_{\frac{1}{2}}\left(T_{2}-T_{1}\right)-A_{1 \mathrm{bc}} q_{1 \mathrm{bc}}\right) / V_{1}\right)+\left(\frac{1}{\rho C_{\rho}}\right)\left(\sum_{1=1}^{J} \mathcal{H}_{j} \rho r_{i}\right) \\
& \left(\frac{\lambda}{\rho C_{\rho}}\right)_{n p t}\left(\left(A_{n p t ~ b c} q_{n p t ~ b c}-\left(\frac{2 \lambda_{n p t}-1}{\lambda_{n p t-1}+\lambda_{n p t}}\right) A_{n p t-\frac{1}{2}}\left(T_{n p t}-T_{n p t-1}\right)\right) / V_{n p t}\right)+\left(\frac{1}{\rho C_{p}}\right)\left(\sum_{n p t}^{J} \mathscr{H}_{j} \rho r_{i}\right)
\end{aligned}
$$

where $A\left(\mathrm{~cm}^{2}\right)$ is the area and $V\left(\mathrm{~cm}^{3}\right)$ is the volume of a computational node and all other quantities have been defined previously. The contribution to the two boundary fluxes are given by:

$$
\begin{aligned}
& A_{1 \mathrm{bc}} q_{1 \mathrm{bc}}=\mathrm{a}_{\mathrm{in}} \times \lambda_{1} \times\left(\frac{T_{\mathrm{in}}-T_{1}}{x_{0}-x_{1}}\right)+\left(1.0-\mathrm{a}_{\mathrm{in}}\right) \times \\
& \left(f_{\text {in }}+h_{\text {in }}\left(T_{\text {in }}-T_{0}\right)+\varepsilon_{\text {in }} \sigma\left(T_{\text {rad, in }}^{4}-T_{0}^{4}\right)\right) \\
& A_{n p t, b c} q_{n p t, b c}=a_{e x} \times \lambda_{n p t} \times\left(\frac{T_{e x}-T_{n p t}}{x_{n p t+1}-x_{n p t}}\right)+\left(1.0-a_{e x}\right) \times \\
& \left(f_{\text {ex }}+h_{\text {ex }}\left(T_{\text {ex }}-T_{\text {npt }+1}\right)+\varepsilon_{\text {ex }} \sigma\left(T_{\text {rad ex }}^{4}-T_{n p l+1}^{4}\right)\right.
\end{aligned}
$$

Cell 0 Cell 1

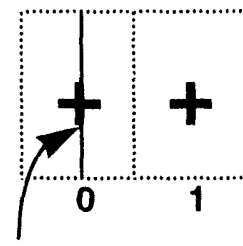

left-hand (inner) boundary
Cell npt Cell npt +1

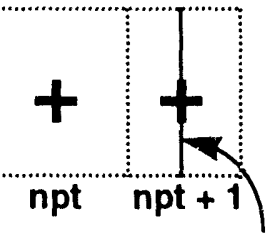

right-hand (outer) boundary

Figure 4. XCHEM node and cell designations 
The $a_{\text {in }}$ and $a_{\text {ex }}$ (dimensionless) parameters determine whether Neumarın (specified variable flux) or Dirichlet (specified time varying temperature) boundary conditions are applied. If $a_{\text {in }}$ or $a_{e x}$ equal " 1 ", then a Dirichlet condition is desired, and its value is fixed by the value of $T_{\text {in }}$ and $T_{\text {ex }}$. If $a_{\text {in }}$ or $a_{\text {ex }}$ equal " 0 ", then a Neumann condition is computed based on a constant term $\left(f_{\text {in }}\right.$ or $\left.f_{\theta x}\right)$, a convective term $\left[h_{\text {in }}\left(T_{\text {in }}-T_{0}\right)\right]$ or $\left[h_{\text {ex }}\left(T_{\text {ex }}-T_{\text {npt }+1}\right)\right]$, or a radiative term $\varepsilon_{\text {in }} \sigma\left(T_{\text {rad, in }}^{4}-T_{0}^{4}\right)$ or $\varepsilon_{\text {ex }} \sigma\left(T_{\text {rad }, \mathrm{ex}}^{4}-T_{\text {npt } 1}^{4}\right)$.

$T_{\text {rad,in }}$ and $T_{\text {rad,ex }}$ are the externally viewed radiation temperatures for the inner and outer boundaries, respectively. The two temperatures, $T_{\text {in }}$ and $T_{\text {ex }}$, are taken as representative time-dependent functions given as:

$$
\begin{aligned}
& T_{\text {in }}=z_{1} \times t \times e^{-t / i n}+T_{\text {in,con }} \\
& T_{\text {ex }}=z_{2} \times t \times e^{-t / t e x}+T_{\text {ex,con }}
\end{aligned}
$$

In Equations (38) and (39), $t$ is time and $t_{\text {in }}$ and $t_{\theta x}(s)$ are time constants that introduce a time dependency on the Dirichlet temperature boundary conditions. $z_{1}$ and $z_{2}$ are nondimensional constant multipliers. (41)

$T_{0}$ and $T_{n p t+1}$ are boundary temperatures that have been linearly extrapolated from their two nearest neighbor nodes as:

$$
\begin{gathered}
T_{0}=T_{1}+\frac{\left(T_{2}-T_{1}\right)\left(x_{0}-x_{1}\right)}{\left(x_{2}-x_{1}\right)} \\
T_{n p t+1}=T_{n p t}+\frac{\left(T_{n p t}-T_{n p t-1}\right)\left(x_{n p t+1}-x_{n p t}\right)}{\left(x_{n p t}-x_{n p t-1}\right)}
\end{gathered}
$$




\section{PART TWO: XCHEM USER'S MANUAL}

\subsection{General Description}

XCHEM consists of fourteen FORTRAN routines. not including the debdf stiff solver. A separate postprocessing graphics driver, called XPLOT, which utilizes the RSCORS Package, is included to aid the user in data interpretation. A diagram of the XCHEM program structure is shown in Figure 5. Input and output files are denoted by a black background. The stiff ODE solver routines have been lumped into a box labelled "debdf". There are actually about thirty routines that comprise the entire debdf solver.

Distribution of the XCHEM code consists of three basic modules: (1) the driver software includes the main calling program, and routines for user input and file manipulation, printing, property data definition, and specification of the ODE's in routine func which is used by the solver; (2) the solver software consists of the routine debdf and all of its auxiliary routines; and (3) the graphics software consists of the XCHEM driver for the plotting software called XPLOT and the plotting RSCORS graphics package, a low-level, machine-portable package developed at Sandia National Laboratories. Included with the graphics package is the PQst Processing POP software, which is the device interface code that allows the graphics to be displayed on a variety of formats for screen or hardcopy display.

XCHEM is written in ANSI Standard FORTRAN 77. There are no FORTRAN "ENTRY" statements in $X C H E M$, which can cause some difficulty with FORTRAN compilers on some computing platforms. On 32bit machines, the accuracy of the ODE solver requires that XCHEM be in double precision. XCHEM units are "cgs" (centimeter, gram, second) and use the energy unit of calorie.

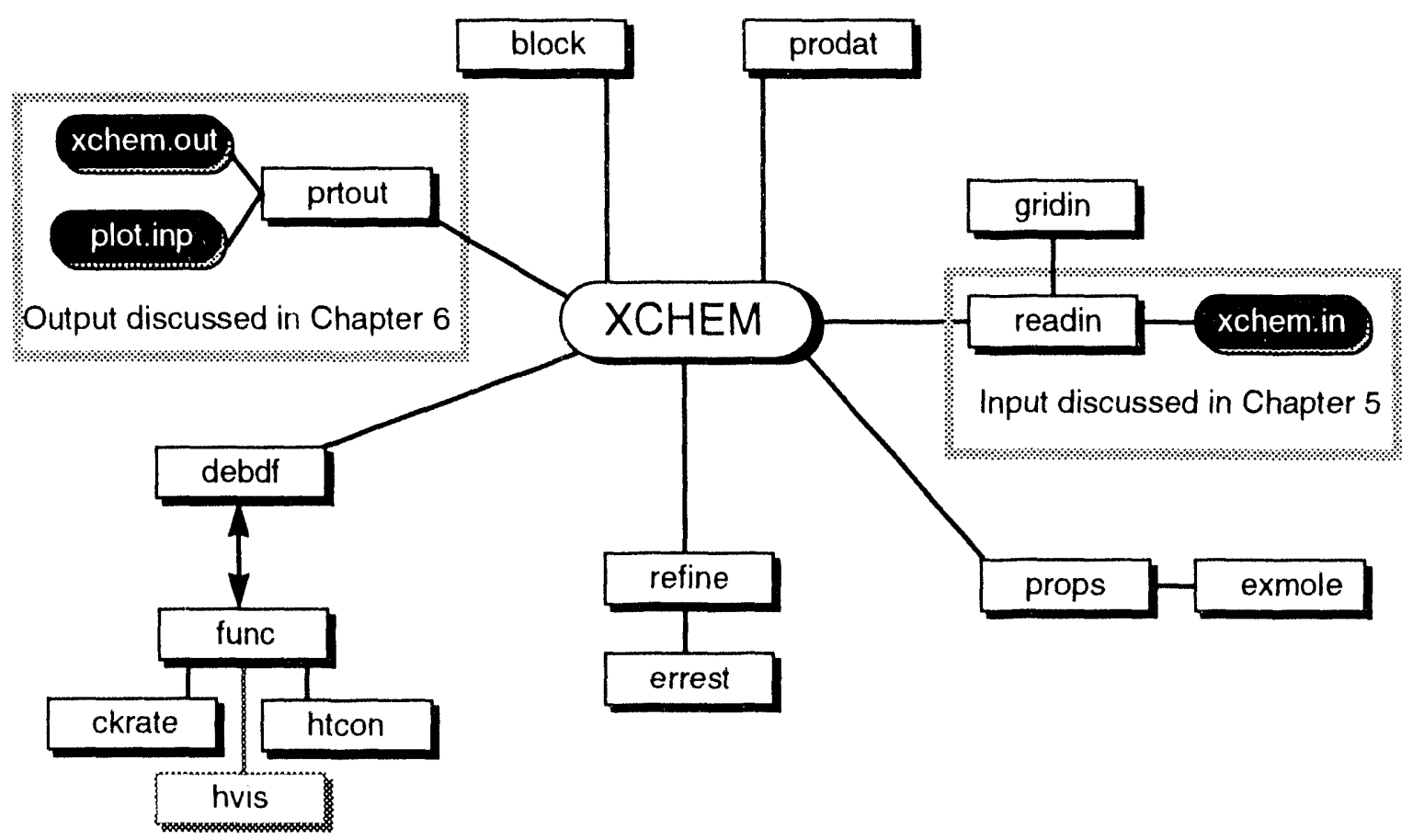

Figure 5. XCHEM program structure 


\subsection{Description of Routines}

The following is a brief description of each of the fourteen routines contained in XCHEM, not including the stiff solver debdf routines. They are, for the most part, listed in the order in which a FORTRAN compiler encounters them when executing XCHEM. The routines for the solver (debdf) ${ }^{15}$ and graphics library (RSCORS) ${ }^{6}$ will not be discussed. A user may examine the XPLOT source code (and the resulting plots that are created) if there is a need to create or customize plots not made by XPLOT.

\section{XCHEM}

Main routine XCHEM first calls subroutine READIN to read data from the user input file $x$ chem.in, and then calls the subroutine PROPS to compute properties of the gas mixture. XCHEM then initializes timestepping parameters, and begins the main timestepping loop. In this loop, the timestep is set, the grid refinement routine is called, geometry variables (which may have been modified by grid refinement) are reset, the number of equations is computed, the solver is called, the dependent variable arrays are reinitialized by the solver, and then printing, plotting, and restarts are performed as necessary.

\section{READIN}

Primarily, subroutine READIN reads in data from the user input file $x$ chem.in. However, READIN also sets some parameters for the ODE solver and the adaptive grid routines. Next, the restart data, the boundary condition data, and the general input parameters (See Appendix A) are read. Contact resitance data, if applicable, is then read, after which the plotting parameters are read. READIN reads in the material data and computes the number of reactant materials and the number of equations in each layer. READIN also calls subroutine GRIDIN to set up the initial grid system. READIN initializes the dependent variable $f$ array, computes pointers to obtain appropriate properties of each energetic matellal in the layer, and also determines printout pointers. READIN computes the time interval for writing restart files, and also reads a restart file to restart a problem if so specified by the user input. READIN also performs some error checking. Layer interface dimensions are checked for consistency. The input parameter, dtmult, is checked (a value greater than 10.0 can decrease accuracy.) If the user includes contact resistance for a one-layer problem, the contact resistance is removed by READIN. READIN will limit the number of restarts to 50 . A check for consistency between the desired start time and finish time is also made. Subroutine READIN is called once by the main XCHEM driver routine.

\section{GRIDIN}

Subroutine GRIDIN sets up the initial grid system for the adaptive grid routines, including all of the spatial arrays. For multi-layered problems, the user may choose a number of gridpoints which produces disparate grid spacings at the interface between two layers. This undesirable condition is corrected in GRIDIN by forcing the aspect ratio between computational cells in adjacent layers to be greater than 0.4 and less than 2.5. GRIDIN inserts nodes in the appropriate layer until this criterion is met. If plotting is desired, GRIDIN prints the initial grid information to the plotting database. GRIDIN is called only once by routine READIN.

\section{PROPS}

Subroutine PROPS computes the gas mixture viscosity and thermal conductivity using the methods discussed in Chapter 2. The gases are constructed from the specification of the initial $\mathrm{CHNOCl}$ (carbon, hydrogen, nitrogen, oxygen, and chlorine) content in the energetic reactant materials. Presently, the gases considered are: $\mathrm{N}_{2}, \mathrm{H}_{2}, \mathrm{H}_{2} \mathrm{O}, \mathrm{O}_{2}, \mathrm{CO}, \mathrm{CO}_{2}, \mathrm{HCl}$, and $\mathrm{Cl}_{2}$. The routine EXMOLE is called from PROPS to compute the number of moles of each constituent gas produced by the quantity of the reactive material. This computation is performed by layers, and each layer may have more than one type of reactive material. Based on the computed gas mixture, PROPS then computes pure component properties, and then 
constant mixture values according to the procedure outlined in Chapter 2. PROPS is called only once by the main driver $\mathrm{XCHEM}$.

\section{BLOCK DATA}

BLOCK DATA is accessed at the beginning of execution. BLOCK DATA contains the CHNOCl composItion of the default energetic materials listed in the XCHEM data library. BLOCK DATA also contains the integer unit numbers for $1 / O$, so a unit number can be changed easily. Subroutine READIN details what unit number corresponds to what $1 / O$ representation, i.e. XCHEM input, XCHEM output, graphics file, etc.

\section{EXMOLE}

Subroutine EXMOLE is called once by PROPS. This small routine determines the number of moles of $\mathrm{N}_{2}$, $\mathrm{H}_{2}, \mathrm{H}_{2} \mathrm{O}, \mathrm{O}_{2}, \mathrm{CO}, \mathrm{CO}_{2}, \mathrm{HCl}$, and $\mathrm{Cl}_{2}$ from the available s sactant materials provided by PROPS.

\section{BLOCK DATA PRODAT}

BLOCK DATA PRODAT contains the property data for both reacting and non-reacting initial materials. For all materials, PRODAT contains the specific heat, thermal conductivity, phase change, latent heat, and density data. For reacting materials, the steric factor, natural logarithm of the pre-exponential factor, activation energy divided by the universal gas constant, energy release for the reaction, concentration exponents, and coefficients on the $j$ reaction rates are also contained in this data base. For both reacting and non-reacting materials, the user may specify a "generic" reacting or non-reacting substance in the xchem.in input file with the appropriate conditional parameters, R6ADD and N6ADD.

\section{REFINE}

Subroutine REFINE regrids the one-dimensional mesh based on relative error estimates computed for each dependent variable for each node by using interpolated error estimates. A cumulative error estimate for each element is obtained by using a weighted sum of the components errors on that node. Node insertion occurs if the error exceeds a preset tolerance. Likewise, node removal can occur if the error is less than another specified tolerance. Further, an automatic smoothness restriction is imposed so that no element can be more than twice as large or less than half as small as its nearest neighbors. REFINE is called within the controlling timestep loop of XCHEM.

\section{ERREST}

Subroutine ERREST is called once by REFINE. ERREST computes the interpolation errors which REFINE uses to determine node insertion or deletion.

\section{FUNC}

Subroutine FUNC defines the coupled ordinary differential equation set given in Equations (31-32). FUNC, utilized by the DEBDF solver, is called in the large timestep loop in the XCHEM main driver. FUNC is usually called ten to one hundred times per timestep, and most of the computing time for XCHEM is spent in FUNC. FUNC also calls subroutine CKRATE and also the function subroutine HTCON.

\section{HTCON}

Subroutine HTCON computes the thermal conductivity of the gas mixture based on the correlations given in Section 2.7. 


\section{HVIS}

Subroutine HVIS computes the viscosity of a gas mixture, and is presently not used by XCHEM.

\section{PRTOUT}

Subroutine PRTOUT prints out data to the XCHEM ASCII data output file, the graphics file to be used as input to XPLOT, and to the XCHEM ASCII restart file. PRTOUT can be called multiple times from the XCHEM timestep loop, but its frequency of use depends on how often the user has specified that printed output, graphics output, and restarts be written to their respective files. 


\subsection{XCHEM User Input}

\subsection{Introduction}

Although XCHEM is a advanced code, using sophisticated adaptive gridding and stiff numerical solvers, a primary objective, as stated in Chapter 1 , was to make XCHEM simple to use. Thus, for simplicity, only one input file is required to run XCHEM, and the file presently must be named $x c h e m$.in. The user should begin with one of the example input files lisied in Appendix A (included in the distribution disks), and then edit them to run their problems of interest. For the most part, there is only one free-format entry on a line followed by a comment that explains that entry in some detail. The input has been divided into six main sections: a header section, restart parameters, bo undary condition parameters, general input parameters, plotting parameters, and material property parameters.

The following sections explain each entry into the xchem. In file. Each entry is tagged indicating whether it is considered required or optional input, and as to the type of input, i.e. character, integer, logical, or real.

\subsection{Header Section}

The header section allows the user to place comments at the beginning of the input to describe the problem of interest.

\section{ncomen (required, integer)}

The number of comments in the header section that immediately follow this entry. This allows the user to internally document the input file. ncomen equal to zero means that after the entry line for ncomen, the comments for the restart parameters immediately follow. ncomen cannot be less than zero.

\subsection{XCHEM Restart Parameters}

\section{Restart Parameters: (required, character)}

The three lines consisting of a line of dashes, the line stating that the following data are "RESTART PARAMETERS" and another line of dashes is required input (See Appendix A).

\section{rstart (required, logical)}

Indicates a desire to read from a restart file. This value must be either ".true." or ".false.".

\section{filnam1 (required, character)}

The name of the restart file from which restart data is to be read. This file must have been previously created by setting the next variable, startw, to "true." and assigning a file name to this write file. The files from which restart files are read from and written to must be kept separate. Even if a restart is not desired, a dummy file name must be entered.

\section{rttime (required, real or integer)}

Specifies the time, in seconds, at which the user desires to restart execution. XCHEM automatically reads the entire restart file, determines the restart closest to this time, and continues execution from this time. An additional teature is that, if the user specifies an rttime value of -1, XCHEM automatically chooses the last time in the restart file.

\section{startw (required, logical)}

Indicates a desire to write a restart file. Again, startw can only be ".true." or "false."

\section{filnam2 (required, character)}

The name of the restart file to which data is to be written. The file names for writing restart files and read- 
ing restart files must be different. Also, even if the user does not want to write a restart file, a dummy file name must be entered.

\section{rwtime (required, real)}

Specifies the time interval, in seconds, at which the user desires to write restart images to filnam2. $X C H E M$ presently allows up to 50 restart images to be written. Based on the beginning and final times that the user specifies in the input, XCHEM determines whether the maximum of 50 images can be exceeded by using rutime. If the maximum will be exceeded, then rutime is recomputed in XCHEM, and a warning message is written to the screen informing the user that rwtime has been adjusted to a value different than that specified in the input

\subsection{XCHEM Boundary Condition Input Parameters}

XCHEM allows the user to specify a variety of boundary conditions at each end of the one-dimensional boundaries and are discussed in detail in Section 3.2. The specification is based on variables that define: a time-dependent boundary temperature; a boundary heat flux, a boundary convection heat transfer coefficient, an emissivity for thermal radiation, and a free field radiation temperature to which to radiate.

The time dependent boundary temperature is specified by:

$$
\begin{aligned}
& T_{e x}=z_{\text {mult1"time }} e^{(- \text {time/texdiv })}+\text { texcon } \\
& T_{\text {in }}=z_{\text {mult2.time }} e^{(- \text {time/tindiv })}+\text { tincon }
\end{aligned}
$$

The user is reminded that the XCHEM code uses units of cgs, so that temperature is Kelvin, heat flux is $\mathrm{caV}\left(\mathrm{sec} \mathrm{cm}^{2}\right)$, and convective heat transfer coefficient is $\mathrm{cal} /\left(\mathrm{sec} \mathrm{cm}^{2} \mathrm{~K}\right)$.

\section{Boundary Condition Parameters: (required, character)}

The three lines consisting of a line of dashes, the line stating that the following data are "BOUNDARY CONDITION PARAMETERS" and another lines of dashes is required input (See Appendix A).

\section{zmult1(required, real)}

Multiplier on the time dependent portion for evaluating the external temperature boundary condition value as shown in the above equation. Also, see Section 3.2 .

\section{texdiv (required, real)}

Coefficient in the exponential term for evaluating the external temperature boundary condition value as defined above.

\section{texcon (required, real)}

Constant term (units of $\mathrm{K}$ ) for evaluating the external temperature boundary condition value as defined above.

\section{fex (required, real)}

Specifies the value of the exterior boundary heat flux in $\mathrm{cal}\left(\mathrm{sec} \mathrm{cm}^{2}\right)$. See Section 3.2.

hex (required, real)

Specifies the value of the exterior heat transfer coefficient in $\mathrm{cal} /\left(\mathrm{sec} \mathrm{cm}^{2} \mathrm{~K}\right)$. The fex value and the hex value multiplied by the temperature difference of $T_{e x}$ and the nearest exterior node are added together to compute a cumulative heat flux on the exterior boundary as described by Equation (37).

\section{esex (required, real)}

Specifies the value of exterior emissivity multiplied by the Stefan-Boltzmann constant in units of caV(sec $\mathrm{cm}^{2} \mathrm{~K}^{4}$ ) which is the $\varepsilon_{\text {ox }} \sigma$ product in Equation (37). 
trex (required, real)

Specifies the external temperature to which the external boundary radiates to complete specification of the boundary condition for radiation.

zmult2 (required, real)

Multiplier on the time dependent portion for evaluating the internal temperature boundary condition value as defined above. Also, see Section 3.2.

\section{tindiv (required, real)}

Coefficient in the exponential term for evaluating the internal temperature boundary condition value as defined on the previous page.

\section{tincon (required, real)}

Constant term (units of $\mathrm{K}$ ) for evaluating the internal temperature boundary condition value as defined on the previous page.

\section{fin (required, real)}

Specifies the value of the interior boundary heat flux in cal/(sec $\left.\mathrm{cm}^{2}\right)$. For systems using cylindrical or spherical coordinates, if the center is the interior, then an adiabatic condition is usually required and this value should be zero. For performing calculation on shell-like geometries, then this value can be nonzero.

\section{hin (required, real)}

Specifies the value of the interior heat transfer coefficient in cal $\left(\mathrm{sec} \mathrm{cm}^{2} \mathrm{~K}\right)$. The fin value and the hin value multiplied by the temperature difference of $T_{\text {in }}$ and the nearest interior node are added together to compute a cumulative heat flux on the interior boundary. Again, for cylindrical and spherical geometries when the interior is located at the center, this value should be zero.

esin (required, real)

Specifies the value of interior emissivity multiplied by the Stefan-Boltzmann constant in units of caV(sec $\mathrm{cm}^{2} \mathrm{~K}^{4}$ ) which is the $\varepsilon_{\text {in }} \sigma$ product in Equation (36).

trin (required, real)

Specifies the internal temperature to which the internal boundary radiates to complete specification of the inner boundary condition for radiation.

alex (required, real)

Boundary condition flag that specifies either a Neumann (flux) or Dirichlet (specified temperature) boundany condition on the node designated as the last node, $n p t$. If alex $=0$, then a Neumann condition is specified. If alex $=1$, then a Dirichlet condition is specified. See Section 3.2 for additional details.

\section{alin (required, real)}

Boundary condition flag that specifies either a Neumann (flux) or Dirichlet (specified temperature) boundary condition on the node designated as the first node, node 1 . If alin $=0$, then a Neumann condition is specified. If alin=1, then a Dirichlet condition is specified. See Section 3.2 for additional details.

\subsection{XCHEM General Input Parameters}

\section{geom (required, real)}

Specifies the geometry type: a value of 0.0 specifies cartesian (slab) geometry; a value of 1.0 specifies cy- 
lindrical geometry; and a value of 2.0 specifies spherical geometry. Note that this is a real-valued parameter since it specifies the metric coefficient for computation of a cell volume with XCHEM.

\section{refit (required, logical)}

Specifies whether grid refinement is desired. Since the grid refinement typically takes less then $5 \%$ of the entire computation time of an XCHEM run, usually this parameter is ".true ", unless simple one node calculations are being performed.

\section{time (required, real)}

The initial starting time in seconds.

\section{timfin (required, real)}

The final time in seconds. For a complete calculation, this time should be larger than the anticipated time for ignition. One approach is to assign a large value to this parameter. Note, however, if the user is using the restart capability, that timfin is used to compute restart time intervals. Also, if the boundary conditions are such that thermal runaway does not occur (e.g. $T<T_{c}$, where the subscript denotes the critical temperature), XCHEM will stop when timfin is reached. Thus, some judgment should be exercised in the choice of timfin.

\section{dtmult (required, real)}

The stiff solver used in XCHEM recommends a new user timestep after every time advancement observed by the user as screen output. It is certainly allowed, and we strongly recomrnend, that this value be increased by the use of dtmult, which is a multiplier on the stiff solver's timestep recommendation. XCHEM grid refinement is explicit from the solver; during a time interval grid refinement occurs only after a time of dtmult times the recommended timestep has passed. Too large a value of dtmult will result in XCHEM not refining the grid during times in which possible physical events which might require refinement can occur. On the other hand, too small a value of dtmult results in large computer cpu times. We recommend a default value of 10 to 15 based on our experience of running a large variety of problems.

\section{dtprnt (required, real)}

The print interval, in seconds, that output to the printed output file is desired. The output file is always named xchem.out. XCHEM, unlike some other codes, will always print output based exactly on this userspecified interval. Sometimes XCHEM takes some small timesteps to achieve the exact printout time, as the user can determine from the screen output.

\section{dtplot (required, real)}

This parameter specifies the interval, in seconds, that output to the plot file is desired. This parameter is required even if plotting is not specified. However, dtplot is not used if plotting is disabled, so in this case any real value is allowed. The plot filename is specified in the PLOTTING PARAMETERS section. As a rough guide, most of the three-dimensional plots shown in this document have been made with between 50 and 60 lines on the plot. Thus, taking the final time (minus the initial time) and dividing by 50 usually provides a good initial value for this parameter.

nlayer (required, integer)

The number of layers in the problem. A maximum of 10 layers is presently allowed.

\section{loc(1) to loc(nlayer+1) (required, real)}

Location of the first layer boundary must be the internal location of layer \#1. The second location value is that of the final location of layer \#1 (which is also the starting location of layer \#2, if present). This would be a complete specification for a one layer problem. A two layer problem would require another entry, for a to- 
tal of three layer boundaries. Three layers requires four entries, and so on. Each entry is on a separate line. Dimensions are $\mathrm{cm}$.

\section{nnode(1) to nnode(nlayer) (required, integer)}

Starting with layer \#1. sequentially specify how many initial nodes are desired in each layer. Again, each entry is on a separate line. Since XCHEM performs automatic grid refinement, these value(s) are not critcal We do not recommend placing over 100 total nodes in the system because there is an overall liınit on number of nodes that XCHEM allows (currently 300 nodes), and it is important to let the grid refinement decide how many nodes are necessary, and where they should be placed. See the example input listings in Appendix A for further guidance

\section{temp(1) to temp(nlayer) (required, real)}

The initial temperature of each layer in Kelvin. Again each entry is on a separate line.

re (required, real)

Relative error for the stiff solver. We have found a value of $1.0 \times 10^{-6}$ to be adequate for a wide variety of problems. Smaller values may be used to achieve convergence and/or greater accuracy on extremely difficult problems, at a commensurately greater cost in computer cpu time. This value should be greater than ae the absolute error. specified next.

ae (required, real)

Absolute error for the stift solver. We have found a value of $1.0 \times 10^{-10}$ to be adequate for a wide variety of problems. A larger or smaller value may be used. This value should be smaller than the relative error, re, discussed above.

\section{ncont (required, integer)}

Number of contact resistances in the problem. If the number of layers is one, then there can be no contact resistance. However, an $1 / O$ check is performed such that if ncont is nonzero and there is only one layer, then ncont is set to zero.

\section{layer\#, layer\# (optional, integer)}

Input on the same line, the numbers of the two layers between which the contact resistance lies. If ncont is larger than one, then input on sequential lines the layers between which these additional resistances lie Thus, three contact resistances for a four-layer problem would require thee lines of data, on which each line were entered a pair of integer values representing layer numbers.

\section{emiss1, omiss2, gapdis, tcf, voidfr (optional, real)}

Five values input on the same line emiss1 and emiss2 are the radiative emissivities of the two surfaces between which the gap lies. If zero values are entered, then XCHEM automatically assigns default values of 1.0 for these two parameters. gapdis is the gap distance in $\mathrm{cm}$. If a zero value is entered, then XCHEM assigns a value of $0.1 \mathrm{~cm}$ to gapdis. tcf is the thermal conductivity of the fluid in the gap, which assumes a value of $7.2 \times 10^{-5} \mathrm{caV}(\mathrm{sec} \mathrm{cm} \mathrm{K})$ if the user enters a zero value. This value is representative of that of air. Finally, voidfr is the percentage of surface area representative of void. voidfr is assigned a value 0.8 in XCHEM if the user enters a zero value for this parameter.

\section{tkill (required, real)}

The criteria which determines that ignition has been reached can be difficult to program. tkill represents one of those criteria for XCHEM. tkill is the lower temperature limit, in Kelvin, that is used in XCHEM to determine whether ignition has occurred. If this temperature has not been reached anywhere in the computational domain, then XCHEM will continue the problem. If tkill has been reached or exceeded, the $X C H E M$ calculation will be terminated. 


\subsection{XCHEM Plotting Parameters}

\section{iplot (required, logical)}

Specifies whether plotting is desired and thus assumes the value of ".true." or "false.".

\section{zzcol (required, logical)}

Specifies whether color is desired for the plots. If zzcol is "false.", then the plots will be in black and white, which may be desirable if only a black and white printer is available for hardcopy.

\section{filnam3 (required, character)}

Specifies the file name to which plot data is written. The default plot file name is plotinp.

\section{title (required, character)}

Specifies the title that will appear on the plots. The length of the title is limited to fifty characters. If plotting is not desired, this line is still required, but any character information, including blanks, can be inserted, as the information will not be used in this case.

\subsection{XCHEM Material Parameters}

With the present release version, the following material parameters are required input in the order in which they are listed. It was initially planned that the user only input the materials of interest to the specific problem, but XCHEM beta users found that having all the materials present to be convenient and desirable.
1 ' $h$ mx' \$ material 1 is HMX
2 'tatb' \$ material 2 is TATB
3 ' $r d x$ ' \$ material 3 is RDX
4 'nt' \$ material 4 is TNT
5 ' $n c$ ' \$ material 5 is Nitrocellulose
6 'genrt' \$ material 6 is generic reactive material
7 'alum' $\$$ material 7 is aluminum
8 'cu' \$ material 8 is copper
9 'be' $\$$ material 9 is beryllium
10 'ti' \$ material 10 is titanium
11 'ss304' \$ material 11 is 304 stainless steel
12 'gennrt' $\$$ material 12 is generic nonreactive material

nentry (required, integer)

Specifies the number of entries (and lines) following nentry that specify layer, material and volume fraction information for each layer.

\section{layer, material \#, volume fraction (required, two integer values, one real value)}

Entries must be sequential by layer (first layer 1 , then layer 2 , etc.). The layer and material number are integer values. The material values must match those presented above for the (at present) twelve default material in the XCHEM database. Volume fractions in each layer should total to one, and are real-valued parameters. One of the most common input errors is to change the number of layers and/or materials, only to neglect to change the value of nentry!

rbadd (required, logical)

Specifies whether the user desires to enter in a complete database for an additional reactive material. If 
r6add is ".true." then all of the parameters following up to the entry for n6add must be properly entered into xchem.in.

imax, jmax (optional, integer)

imax is the number of species for the reaction; jmax is the number of reactions.

species, state (optional, integer, character)

There must be imax number of lines for this data field. The species should be sequentially numbered, starting at 1 (integer value), and the state must be either "solid", or "gas". This information is used to determine gas mixture density.

$\mathrm{C}, \mathrm{H}, \mathrm{N}, \mathrm{O}, \mathrm{Cl}$ Composition (optional, real)

These entries, all on one line, represent the proportions of carbon, hydrogen, nitrogen, oxygen, and chlorine in the initial reactive material.

specific heat data - initial phase (optional, real)

Four entries (on one line) which specify the temperature dependent value of specific heat of the reactive material as an initial phase. The first and second entry correspond to the specific heat $(\mathrm{cal} / \mathrm{g} \mathrm{K})$ at the temperatures $(K)$ given by the third and fourth entries, respectively.

specific heat data - second phase (optional, real)

Four entries (on one line) which specify the temperature dependent value of specific heat of the reactive material as a possible second phase, e.g. as a liquid. If a second phase does not exist, then these entries are identical to those of the initial phase. Again, the first and second entry correspond to the specific heat $(\mathrm{cal} / \mathrm{g} \mathrm{K})$ at the temperatures $(\mathrm{K})$ given by the third and fourth entries, respectively.

thermal conductivity data - initial phase (optional, real)

Four entries (on one line) which specify the temperature dependent value of thermal conductivity of the reactive material as an initial phase. The first and second entry correspond to the thermal conductivity (call $\mathrm{cm}-\mathrm{s}-\mathrm{K}$ ) at the temperatures $(\mathrm{K})$ given by the third and fourth entries, respectively.

\section{thermal conductivity data - second phase (optional, real)}

Four entries (on one line) which specify the temperature dependent value of thermal conductivity of the reactive material as a possible second phase, e.g. as a liquid. If a second phase does not exist, then these entries are identical to those of the initial phase. Again, the first and second entry correspond to the thermal conductivity $(\mathrm{cal} / \mathrm{cm}-\mathrm{s}-\mathrm{K})$ at the temperatures $(\mathrm{K})$ given by the third and fourth entries, respectively.

phase change temperature, latent enthalpy, density (optional, real)

Three entries specifying phase change temperature $(K)$, latent enthalpy $(\mathrm{cal} / \mathrm{g})$, and density $(\mathrm{g} / \mathrm{cc})$.

\section{steric coefficients (optional, real)}

Specify $j$ entries on a single line for the steric coefficients, $\beta_{\mathrm{j}}$, for the generic reaction mechanism consistent with the form of Eq. (5).

natural logarithm of pre-exponential factors (optional, real)

Specify $j$ entries on a single line for the natural logarithm of the pre-exponential factors $\left(\operatorname{ln~} \mathrm{s}^{-1}\right)$ for the generic reaction mechanism consistent with the form of Eq. (5).

activation energy divided by the universal gas constant (optional real)

Specify $j$ entries on a single line for the activation energy divided by the universal gas constant $(K)$ for the generic reaction mechanism consistent with the form of Eq. (5). 
energy releases (optional, real)

Specify $j$ entries on a single line for the energy release, $\mathcal{H}_{4}(\mathrm{caVg})$ for the generic reaction mechanism consistent with the form of Eq. (2).

concentration exponents (optional, real)

Specify $i$ lines with each line corresponding to $i$ entries for the concentration elements, $\mu_{i, j}$, consistent with Eq. (4). See Appendix C for examples.

stoichiometry coefficients (optional, real)

Specify $j$ lines with each line corresponding to 1 entries for the stoichiometric coefficients, $v_{i, j}$, consistent with Eq. (6). See Appendix $C$ for examples.

n6add (required, logical)

Specifies whether the user desires to enter in a complete database for an additional inert material. If n6add is "true." then all of the parameters following must be properly entered into xchem.in.

specific heat data - initial phase (optional, real)

Four entries (on one line) which specify the temperature dependent value of specific heat of the inert material as an initial phase. The first and second entry correspond to the specific heat (cal/g K) at the temperatures $(K)$ given by the third and fourth entries, respectively.

\section{specific heat data - second phase (optional, real)}

Four entries (on one line) which specify the temperature dependent value of specific heat of the inert material as a possible second phase, e.g. as a liquid. If a second phase does not exist, then these entries are identical to those of the initial phase. Again, the first and second entry correspond to the specific heat (call $\mathrm{g} \mathrm{K}$ ) at the temperatures $(\mathrm{K})$ given by the third and fourth entries, respectively.

thermal conductivity data - initial phase (optional, real)

Four entries (on one line) which specify the temperature dependent value of thermal conductivity of the inert material as an initial phase. The first and second entry correspond to the thermal conductivity (cal $/ \mathrm{cm}$ $s-K)$ at the temperatures $(K)$ given by the third and fourth entries, respectively.

\section{thermal conductivity data - second phase (optional, real)}

Four entries (on one line) which specify the temperature dependent value of thermal conductivity of the inent material as a possible second phase, e.g. as a liquid. If a second phase does not exist, then these entries are identical to those of the initial phase. Again, the first and second entry correspond to the thermal conductivity (calcm-s-K) at the temperatures $(K)$ given by the third and fourth entries, respectively.

phase change temperature, latent enthalpy, density (optional, real)

Three entries specifying phase change temperature $(K)$, latent enthalpy $(\mathrm{caV} / \mathrm{g})$, and density $(\mathrm{g} / \mathrm{cc})$ for the inert material. 


\subsection{XCHEM Output}

\subsection{XCHEM Screen Output}

XCHEM, like most codes, prints output to the screen as it runs to continually update the user on the code's progress. A partial listing of this output is shown in Figure 6. The XCHEM logo is first printed, and then a welcome which prints out the version number. If the user input is successfully read in, a message indicating the completion of reading in the data file $x c h e m$.in is then printed. At this point, there are two basic outputs to the screen, one which gives information on every XCHEM timestep, and one which informs the user when a grid refinement has occurred. The timestep information includes a flag which indicates the status of the ODE solver, the current time, the current timestep, and the total number of ODE's which $X C H E M$ is currently solving. The latter, of course, depends on the number of reactive materials in the problem and the number of gridpoints. Most of the time, XCHEM will complete a timestep in normal fashion and the ODE flag is " 3 ". If something unusual occurs, the flag will have a negative value, such as " -1 " or "-7". XCHEM has built-in logic which attempts to continue to advance the solution if this occurs; a negative flag values does not necessarily imply that something "bad" has occurred, or that inaccurate answers will occur from this point. In the region of time near ignition, the flag may become negative several times. The primary criterion that XCHEM uses to determine ignition is when the ODE indicator becomes "- 1 " three times in a row. The grid refinement message indicates that a refinement has occurred in a certain layer, and prints out the new number of gridpoints, the number inserted and the number deleted in the course of this refinement.

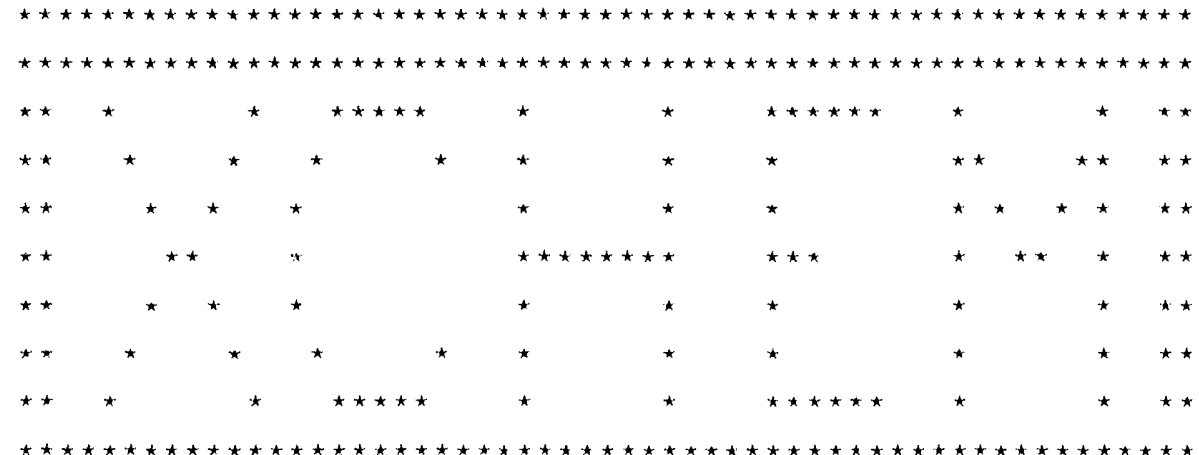

Welcome to $Y$ CHEM version 1.0

Successful completion of user input

ODE ID $=3$ Time $=0.50000 E-02$ Timestep $=0.50000 E-02$ Number eqns $=200$

Refine: New \# nodes in layer $1=61$ Nodes inserted $=11$ Nodes deleted $=0$

ODE ID $=3$ Time $=0.12634 \mathrm{E}-01$ Timestep $=0.76341 \mathrm{E}-02$ Number eqns $=244$

ODE ID $=3$ Time $=0.20264 \mathrm{E}-01$ Timestep $=0.76296 \mathrm{E}-02$ Number eqns $=244$

Refine: New \# nodes in layer $1=68$ Nodes inserted $=7$ Nodes deleted $=0$ ODE ID $=3$ Time $=0.35529 \mathrm{E}-01$ Timestep $=0.15265 \mathrm{E}-01$ Number eqns $=272$

Refine: New \# nodes in layer $1=71$ Nodes inserted $=3$ Nodes deleted $=0$ ODE ID $=3$ Time $=0.51304 \mathrm{E}-01$ Timestep $=0.15776 \mathrm{E}-01$ Number eqns $=284$

Refine: New \# nodes in layer $1=76$ Nodes inserted $=5$ Nodes deleted $=0$ ODE ID $=3$ Time $=0.76951 \mathrm{E}-01$ Timestep $=0.25646 \mathrm{E}-01$ Number eqns $=304$

Refine: New \# nodes in layer $1=84$ Nodes inserted $=8$ Nodes deleted $=0$ 


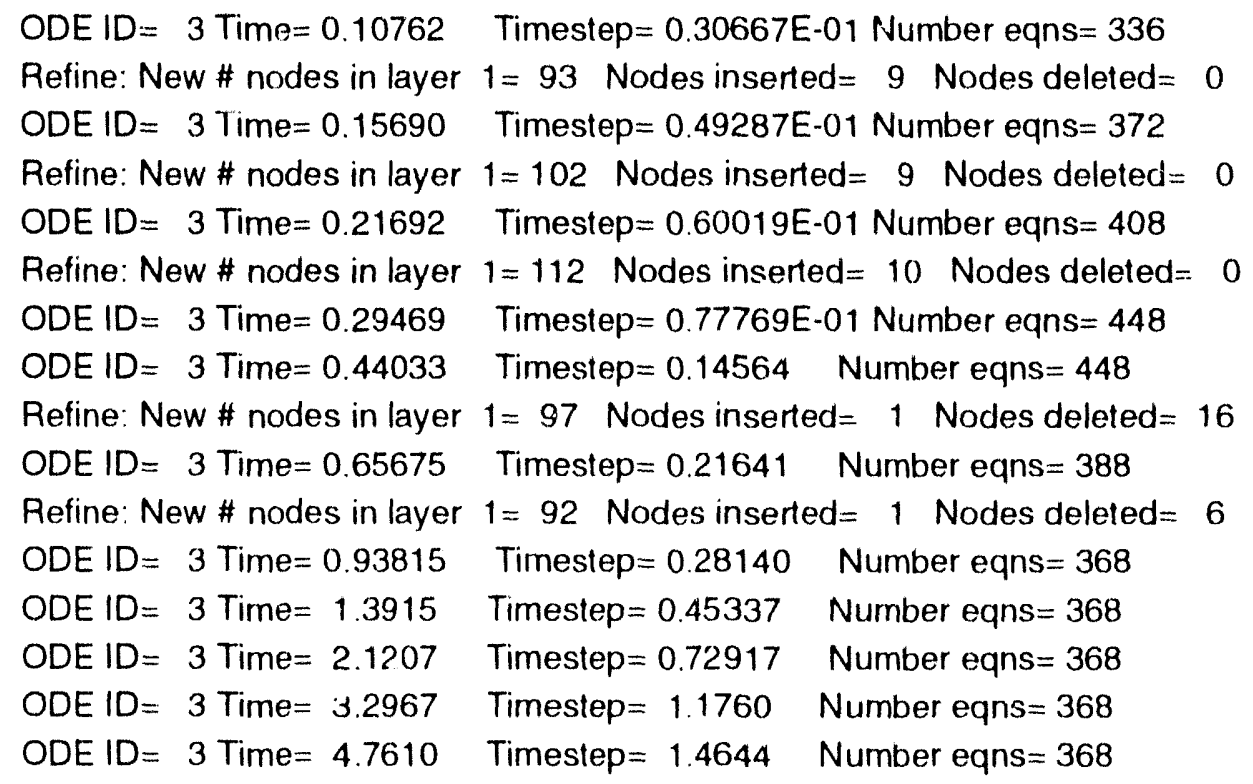

Figure 6. Partial Listing of XCHEM Screen Output

\subsection{Output Files xchem.out and plot.inp}

The default name of the XCHEM ASCII output file is xchem.out. Several examples of this output are shown in Appendix A. The user is able to specify the frequency (by time) of the printout. For each time, the time is printed, and then in a columnar format the distance, temperature, and species concentration of the original reactive materials present at that grid are printed out. Intermediate and final species concentrations are not printed out, although they can be plotted.

The second XCHEM ASCII output file is the database for plotting, which has a default narne of plot.inp. It is not intended for the user to examine this file, so the data is in a cryptic form. Rather, it is intended that XPLOT read this file and use it to create the plots that should prove useful in interpreting XCHEM results.

\subsection{XPLOT and its Output}

XPLOT provides the option for plotting: (1) the original user-specified grid system; (2) a three-dimensional time-space-temperature plot; (3) three dimensional species plots; (4) a two dimensional color fringe plot of temperature; and (5) a projection of the grid system with respect to time. An example of the XPLOT/User interchange is shown in Appendix B. Besides answering questions which determine the particular plots that the user desires, there are also questions which determine whether the user desires either self-scaling plots or to specify certain scales himself. An attempt was made to minimize and simplify the interface with XPLOT to the user. Part of the XCHEM $x$ chem in data file contains information pertinent to XPLOT, including the title for each plot, whether color plots are desired and the time interval between lines in the three-dimensional plot.

A problem with creating the plots arises because the time of ignition is unknown. Although XPLOT selfscales the time plane which eliminates second-guessing the final plot time, the time interval between lines must be known beforehand. Usually 50-60 time lines presents a good plot. If the user guessed ignition to occur at about 250 seconds for a particular problem and thus specified 5 seccnds between plot lines, and the ignition instead occurred in 100 seconds, only 20 time lines would be plotted, giving the plot a sparse appearance. Thus. obtaining the desired plots can be an iterative procedure, requiring that the problem be 
run more than once. Fortunately, XCHEM is efficient and rerunning cases does not present any difficulty.

Figure 7 shows the plot of the grid system. The system as entered by the user and as adjusted by the routine gridin are both shown. As discussed in Section (4.1), one function of routine gridin is to assure that a smooth transition in gridding occurs between layers. Users are prone to grid adjacent layers without assuring that this smooth gridding occurs to avoid numerical inaccuracy or discontinuities at layer interfaces, nor should they be concerned with this detail. Routine gridin instead performs this function. This plot provides a check for the user to assure that dimensions, layers, and gridding were properly entered.

Figure 8 shows a typical time-space-temperature plot from XPLOT. Each line was plotted from space/temperature data at a certain time. The times at which the lines are plotted are specified in $x c h e m$.in. Different layers are shown by different line colors. Figure 9 shows an example of a time-space-species plot from XPLOT. The time between lines is identical to that of the time-space-temperature plot

Figure 10 shows a sample color fringe temperature plot. The color spectrum uses blues to represent cooler colors and then transitions through green, yellow, and orange colors until the hottest color representation, red, is reached.

Figure 11 shows the time-dependence of the adaptive grid system. Again, the time between the lines is identical to that of the previous plots. This is essentially a two-dimensional plot projected from a three-dimensional perspective. This perspective was chosen because it matches the perspective of the three-dimensional temperature and species plots, and the color fringe plot.
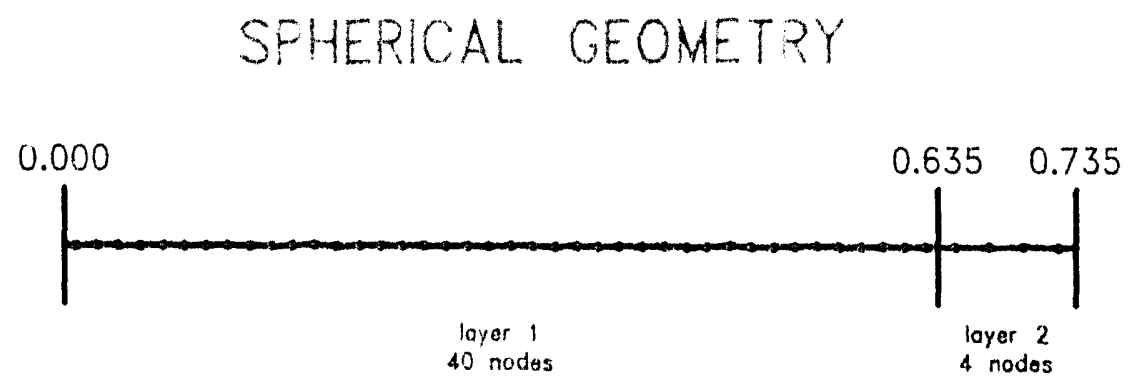

INITIAL GRIDPOINT LOCATIONS

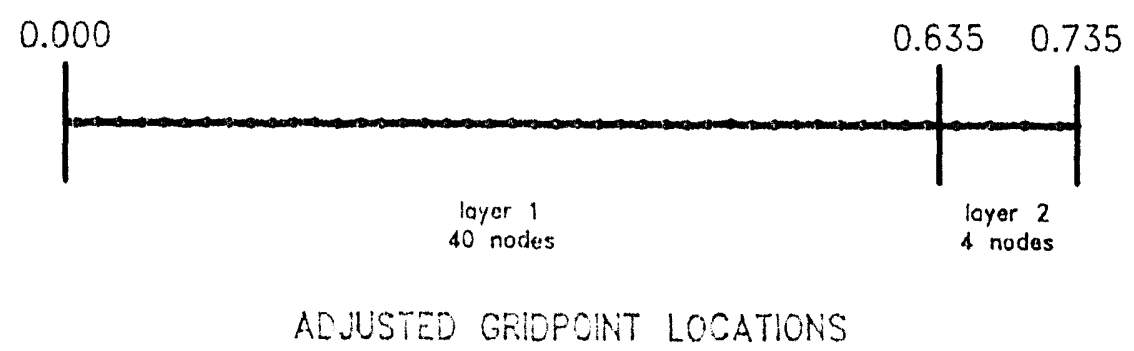

Figure 7. Plot of the initial and adjusted XCHEM grid system 


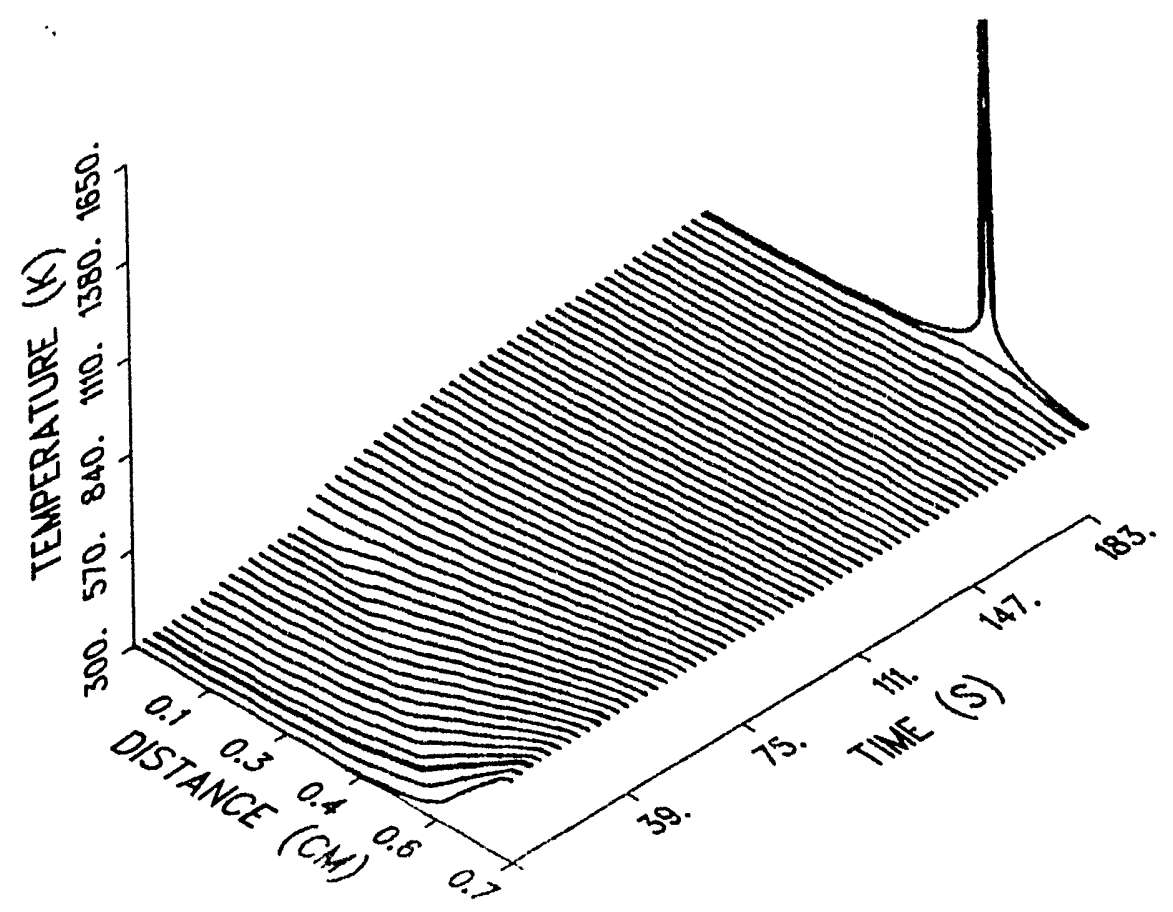

Figure 8 A typical time-space-temperature plot from XPLOT.

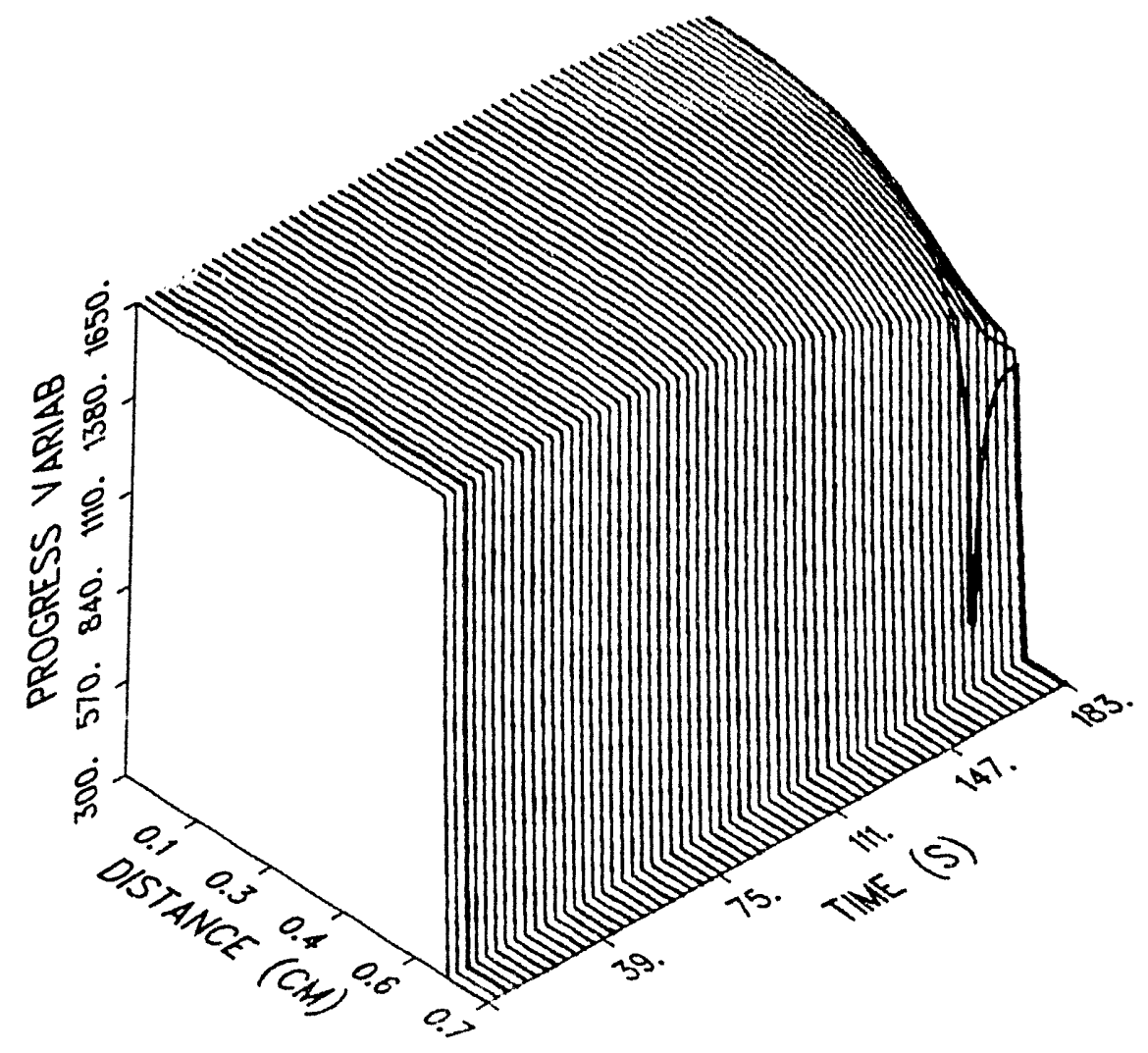

Figure 9 A typical time-space-species plot from XPLOT. 


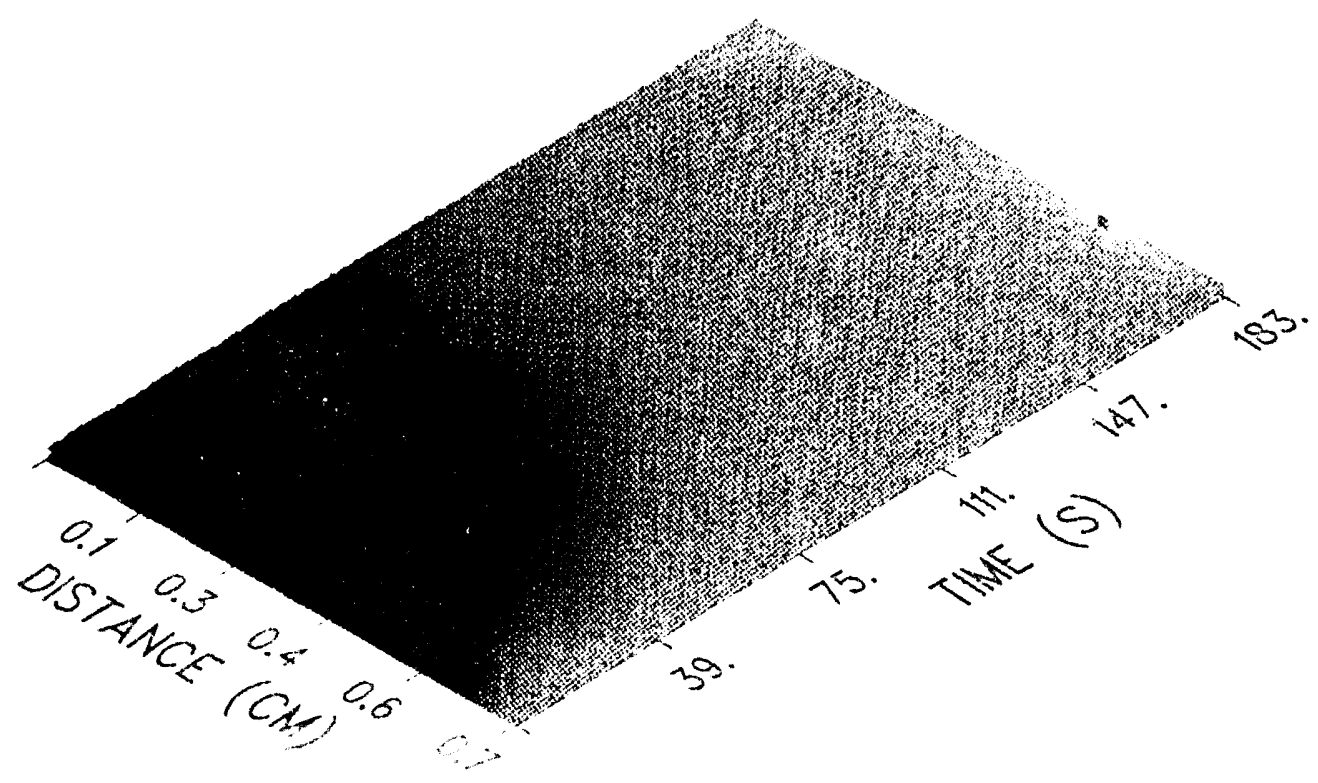

Figure 10. A sample color fringe plot from XPLOT.

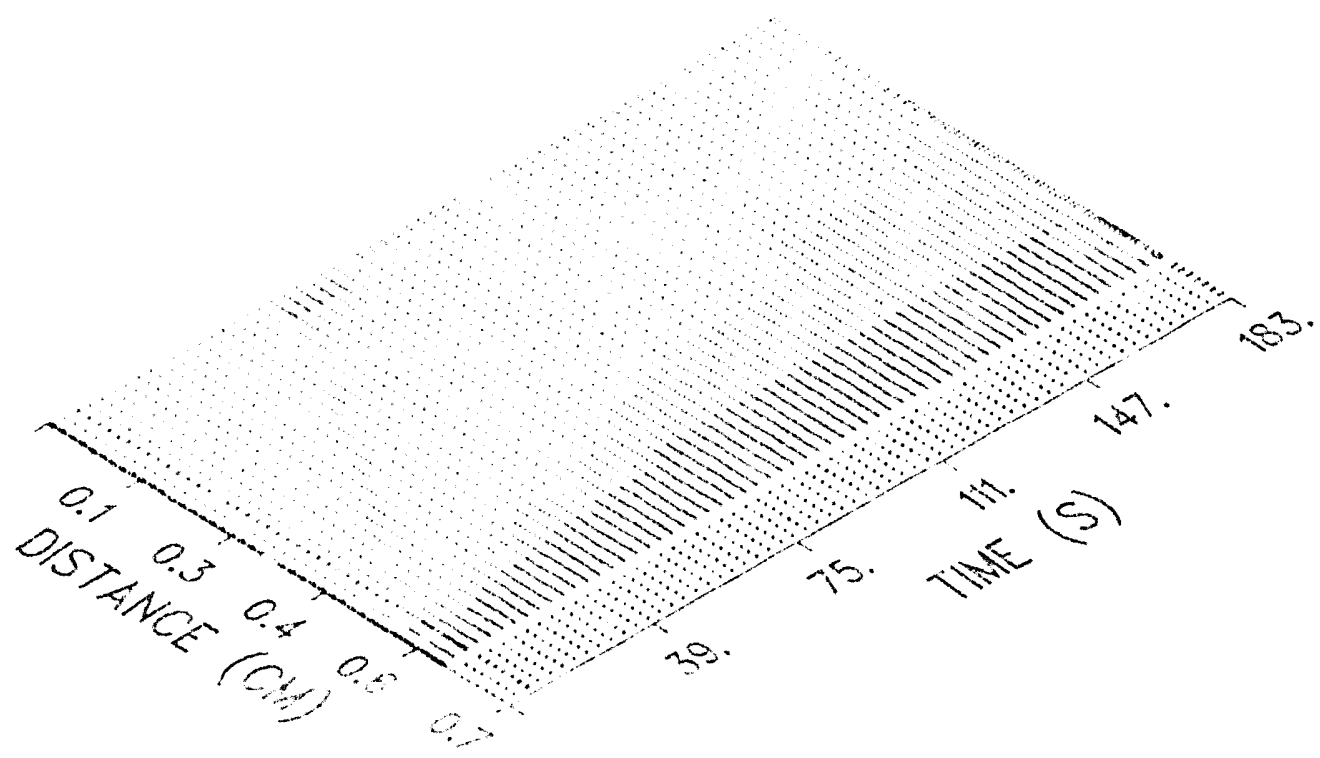

Figure 11. A sample time dependent grid plot from XPLOT. 


\subsection{Installation of XCHEM}

This chapter describes the installation of the three software packages needed for a complete installation of the XCHEM package. These three packages are: (1) the CMP software which is used as a utility to install the RSCORS graphics system; (2) the RSCORS graphics system; and (3) the XCHEM code itself, along with the stiff solver and XPLOT, a driver for the RSCORS graphics package specifically written to interface with the XCHEM database to provide a post-processing capability. In all cases, source has been made available to the user. Most of the installation deals with the installation of the graphics package, so if graphics are not desired, installation of the XCHEM code is trivial.

The RSCORS graphics system was chosen because source code was available, it installs on a wide variety of popular scientific computing platforms, and Sandia National Laboratories has distribution rights for the software. Although it is a low-level graphics system. it has powerful two- and three-dimensional graphics capabilities, as well as a color capability. RSCORS plots can be plotted on a large variety of devices. including both on PC and UNIX-based workstations platforms, and on a variety of hardcopy devices, both color and black and white. A driver for RSCORS. XPLOT, has been included with the XCHEM software to provide some default plotting capability to the user (including three dimensional temperature plots, and color fringe plots), and to serve as an example of how to write a driver if additional graphics are required by the user.

\subsection{Installation on UNIX Systems}

Presently, XCHEM is distributed on five PC floppies. These floppies enable the user to install XCHEM on most platforms available, from the CRAY to the PC. The PC installation requires a special approach, so some of the directories in the installation do not need to be copied for UNIX systems. The directories that need not be copied are:

$$
\begin{aligned}
& \text { /rscdist } \\
& \text { /rsclahey } \\
& \text { /cmpdist } \\
& \text { /rsclibs. }
\end{aligned}
$$

Thus, do not be concerned when this discussion does not mention installation of these subdirectories

\section{Directory Structure}

Three subdirectories should be created along a chosen branch of the directory hierarchy. These three subdirectories should be named:

/xchem

/rscors

/sltutil

The CMP related programs are in /sltutil, the RSCORS related codes are in /rscors, and the XCHEM and XPLOT software is in /xchem.

\section{Copying the Files:}

Copy the two floppies that are designated "RSCORS \#1" and "RSCORS \#2" into the /rscors subdirectory. Copy the one floppy that is designated "SLTUTIL" into the /sltutil subdirectory. Another floppy designated "XCHEM" should be copied into the subdirectory called/xchem. Copy the files from this disk into the /xchem subdirectory on your system. The same floppy has some examples. For each example, there is 
the XCHEM input file, the XCHEM output file, and the plot file that XPLOT uses as input for graphics. You may optionally copy these examples into your XCHEM subdirectory.

\title{
Create the CMP utlility executables
}

These executables must be created first because they are used in the installations following the CMP installation. Go into the /sltutil subdirectory and type, for example:

$\begin{array}{ll}\text { musltutl.sun } & \text { SUN Systems } \\ \text { musitutl.ucs } & \text { UNICOS Systems } \\ \text { musitutl.hpw } & \text { HP Systems } \\ \text { musitutl.i60 } & \text { IBM RISC Systems } \\ \text { musltutl.utx } & \text { DEC ULTRIX Systems }\end{array}$

Type only one of the above commands based on the particular computing platform you are using. The acronym "mu" stands for "make utility" and is used throughout the CMP and RSCORS fiie names. You may need to change permission on the procedure file you are executing, such as:

chmod $+x$ musitutl.sun

After the executables have been created, set the path in your cshrc file, such as:

set path $=(. \sim$ \$path /usr/locaVetc /bin /home/rjgross/sltutil/home/rjgross/rscors /home/rjgross/xchem)

\section{Create the RSCORS executables}

Go into the /rscors subdirectory and type, for example:

murscors.sun
murscors.ucs
murscors.hpw
murscors. 60
murscors.utx

\author{
SUN Systems \\ UNICOS Systems \\ HP Systems \\ IBM RISC Systems \\ DEC ULTRIX Systems
}

Again, type only one of the above commands based on your computing platform and ensure that you have permission to execute the file. Also, after the RSCORS library has been created, place the following in your ".cshrc" files:

$$
\text { setenv RSCORS ../path/librscors.a }
$$

where "../path" represents the directory path where the librscors.a file is located.

In your .profile file, place the following:

$$
\begin{aligned}
& \text { RSCORS = ../path/librscors.a } \\
& \text { export RSCORS }
\end{aligned}
$$

You should logout and then login to get these symbol and paths defined.

\section{Create the RSCORS device drivers}

Again, in the /rscors subdirectory, type: 
mupopx11.xxx

mupop.xxx

where " $x x x "$ is the three letter designator for the platform, i.e. "sun" for SUN systems, "hpw" for HP systems, etc. These make utilities will create the so-called "pop" executables. These are the various device drivers that enable you to put the RSCORS graphics files, called metafiles, onto various screens or hardcopy devices.

\section{Create the XCHEM executable}

Go into the /xchem subdirectory. It should contain three files: xchem.for, solver.for, and xplot.for. Compile and link xchem.for and solver.for together using the Fortran compiler on your machine. For example, on SUN machines, type:

$$
177 \text {-fast -onetrip -o xchem xchem.for solver.for. }
$$

The "-onetrip" option forces all do-loops to be executed at least once. Use of this option is highly recommended. The "-fast" merely disables IEEE checking and allows the code to run faster. The above command will create the executable file named "xchem" with no extension.

\section{Create the XPLOT executable}

Again, in the /rscors subdirectory, create the XPLOT executable by compiling xplot.for and linking it to the RSCORS graphics library, librscors.a. For example, on SUN platforms, this is accomplished by typing:

f77 -o xplot xplot.for \$RSCORS

On, the IBM RISC systems, this is,

$$
\text { xif -o xplot xplot.for \$RSCORS }
$$

the above command creates an executable file named "xplot" with no extension. Some computer systems insist that the Fortran files have a ". $f$ " file extension rather than the ".for" used in these example. If so, then simply rename these three files with the ".f" extension and use these names in the compile and link commands.

\subsection{Installation on PC Systems}

Presently, XCHEM is distributed on five PC floppies. These floppies enable the user to install XCHEM on most platforms available, from the CRAY to the PC. The PC installation requires a special approach that is different from that of UNIX installations. Further, a Lahey Fortran Compiler, version 5.01, or later, is mandatory for this installation.

\section{Directory Structure}

It is recommended that two subdirectories should be created within a chosen branch of the directory hierarchy. These subdirectories may even be connected to the home directory. These two subdirectories should be named:

\section{behem}

Isltutil

Underneath the Isltutil subdirectory, create five additional subdirectories named:

Irscors 


$$
\begin{aligned}
& \text { Irscdist } \\
& \text { Irsclahey } \\
& \text { Icmpdist } \\
& \text { Irsclibs }
\end{aligned}
$$

The CMP related programs are in /situtil, the RSCORS related codes are in rscors, and the xchem and xplot software is in /xchem.

\section{Copying the Files:}

Copy the two floppies that are designated "RSCORS \#1" and "RSCORS \#2" into the Isltutillrscors subdirectory. Copy the one floppy that is designated "SLTUTIL" into the Isltutil subdirectory. Another floppy has the xchem information in a subdirectory called lxchem. Copy this subdirectory into the lxchem subdirectory on your system. Another floppy has some examples in a lexample subdirectory. These examples include the XCHEM input file (there is only one input file), the XCHEM output file, and the plot file that XPLOT uses as input for graphics. You may optionally copy this floppy into your XCHEM subdirectory.

Finally copy the four additional subdirectories from the installation floppy subdirectories of Isltutil, and name them Isltutinrscdist, Isltutinrsclahey, Isltutincmpdist, and Isltutinrsclibs subdirectories.

\section{Create the CMP utility executables}

These executables must be created first because they are used in the installations following the CMP installation. Go into the Isltutil subdirectory and type,

sltinst,

which is a general utility that will install all of the necessary CMP and RSCORS executables on a PC. Choose option \#4, "Compile CMP files with Lahey compiler", and the sttinst utility will automatically complete the CMP installation.

\section{Create the RsđORS executables}

Go into the Isltutil subdirectory and type,

$$
\text { sltinst, }
$$

and choose option \#6, "Compile RSCORS files with Lahey compiler", and the sltinst utility will automatically complete the RSCORS graphics installation.

\section{Create the proper paths in the autoexec.bat file}

Put the following paths in the autoexec. bat file:

.... pathlsitutinrsclahey

.... pathlsitutinrsclibs

.... .pathisltutincmpexes

\section{Create the RSCORS device drivers}

For the PC, the two present available drivers (popvga.exe, and popcga.exe) are already loaded into the Isltutinrsclibs subdirectory. Since you have already set the proper path (or you can load the executables directly into the work directory to use them), no futher action is necessary. 


\section{Create the XCHEM executable}

Go into the Ixchem subdirectory. It should contain three files: xchem.for, solver.for, and xplot.for. Compile and link xchem.for and solver.for together using the Lahey Fortran compiler.

\section{Create the XPLOT executable}

Again, in the Irscors subdirectory, create the XPLOT executable by compiling xplot.for and linking it to the RSCORS graphics library, Irscors. lib. There is a batch file called Irscors. bat located in Isltutinrsclahey that performs this function. Simply type.

$$
\text { Irscors xplot }
$$

and xplot.exe will be created. 


\subsection{Implementation of XCHEM and XPLOT}

\subsection{Roadmap for a Quick Start}

This section is designed as a path for those interested in getting started with XCHEM and XPLOT as quickly as possible First, XCHEM and XPLOT must be installed on the system of interest. Chapter 7.0 explains how to install the software on various systems. Once installation is complete, obtain a sample $X C H E M$ input file that is included with the XCHEM distribution, and rename (or copy) the file:- $x$ chem.in. Then, read the next section, Section 8.2, or how to $r$ in XCHEM and XPLOT.

If difficulties are encountered in runting XCHEM, Appendix A shows several examples of correct input files. If XCHEM terminates running with an error, the most likely problem is that the input deck is missing (or has too much) information. For example, if two layers are specified, then XCHEM requires three interface locations.

If difficulties are encouiered in running XPLOT, Appendix B shows several examples of the proper user interaction with XPLOT. The most common difficulty occurs in grasping the concept of a "metafile" and the fact that plotting is a iwo step process: (1) Running XPLOT to create a "metafile", and (2) Running the POP software (See Chapter 7 ) to view the plots on an arbitrary screen device or to create a hardcopy file to send to a printer (erther color or black and white).

\subsection{Rur,ning XCHEM and XPLOT}

Use of XCHEM is simple. Once the input file (See Appendix A) xchem in has been created, simply type:

$$
\text { xchem }
$$

and examine the screen output (See Figure 6) to follow the progress of the code.

XCHEM creates two output files: xchem.out and plot.inp. The former file is the ASCll output file which displays temperatures and initial species versus distance at user-specified times. The latter file has written the database for plotting using XPLOT.

To use XPLOT, simply type,

$$
\text { xplot }
$$

XPLOT will ask some questions (See Appendix B) concerning plot scaling (automatic scaling is an option), and which plots are desired. Execution time is on the order of seconds on all computational plattorms. XPLOT creates an RSCORS graphics output file, calied a metafile, that can now be used to display. The format of the display software is:

popxxx filename met,

where " $x x x$ " is the device, and the filename in this case is "xplot". Examples of valid graphics devices are:

$\begin{array}{ll}\text { X11 } & \text { Xwindows (for most workstations) } \\ \text { X12 } & \text { Xwindows (for most workstations) } \\ \text { TK4 } & \text { Tektronix } 4014 \\ \text { T05 } & \text { Tektronix } 4105 \\ \text { T15 } & \text { Tektronix } 4115 / 4125 \\ \text { alp } & \text { Alphanumeric } \\ \text { VGA } & \text { VGA Color Graphics (for PC) }\end{array}$




\section{CGA CGA Color Graphics (for PC)}

For workstations, it is strongly recommended that you use the $X$ window drivers to plot on-screen. If you have not used $X$ windows, we suggest that you get help from your system manager. We have yet to find two systems that had Xwindows installed in exactly the same way. Included in mupopx11.XXX is the best guess as where to look for the libraries and " $\mathrm{C}$ " include blocks on your system, but changes might be required. If you must get plots out immediately, use poppst.xxx. This driver creates a postscript output file called popout.pst which you can directly print to black and white hardcopy postscriptprinters.

The pop software will offer the user a choice of screen tormats (rectangular produces the largest plots, while square is useful for papers). Then, it will inquire as to which "frame" the user desires. For begınners, just type " $n<$ ret>" for "next and carriage return" until you feel comfortable with the pop software. On-line help is available. The pop software is very powertul, allowing the user to plot multiple plots on one page, and to edit particular plot from large plot files to create smaller plot files containing only the desired plots from the large file. 


\subsection{References}

1 Memorandum entitled "Thermal Explosion Method of Lines Solver", from M. Baer to A. C Ratzel, dated, February 5, 1992. See Also: Hobbs, M.L., Baer, M.R., and Gross, R.J., "Modeling Ignition Chemistry," JANNAF Systems Hazards Meeting. Fort Lewis, Washington (May 10-14, 1993).

2. Jaeger, D.W., "EXPLO: Explosives Thermal Analysis Computer Code," Los Alamos National Laboratory Report LA-6949-MA, Revised, Los Alamos. New Mexico (September, 1981).

3. Nichols, A.L, "Chemical TOPAZ: Modifications to the Heat Transfer Code TOPAZ: the Addition of Chemical Reaction Kinetics and Chemical Mixtures." Lawrence Livermore National Laboratory Report UCID-20824, Livermore, California (June 7, 1990).

4. Schiesser, W.E., The Numerical Method of Lines, Academic Press, Inc., San Diego, California (1991)

5. Hyman, J. M., "The Method of Lines solution of Partial Differential Equations," Courant Institute of Mathematical Sciences, New York University, COO-3077-139, October, 1978. See Also: LeVeque, R.J. Numerical Methods for Conservation Laws, Second Edition, Lectures in Mathematics, ETH Zürıch, Basel, Germany (1992).

6. Thompson, S.L." "The RSCORS Graphics System," Sandia National Laboratories Report, To Be Published. Draft Distributed with RSCORS Software

7. McCuire, R.R. and Tarver, C.M., "Chemical Decomposition Models for the Thermal Explosion of Confined HMX, TATB, RDX, and TNT Explosives," Seventh Symposium (International) on Detonation, NSWC MP 82-334, 56 (1981).

8. Yao, L.S. and Prusa, J., "Melting and Freezing," Advances in Heat Transfer, 19, Hartnett, J.P. and Irvine, T.F. Jr., eds., 1 (1989).

9. FIDAP User's Manual, Section 8.3, Revision 6.0. Fluid Dynamics International, Inc., Evanston, Illinois (April, 1991).

10. Reid, R.C., Prausnitz, J.M., and Sherwood, T.K., The Properties of Gases and Liquids, Section 10-2, Third Edition. McGraw-Hill Book Company, New York (1977).

11. ibid., Section 9-4.

12. ibid., Appendix C

13. ibid., Section 10-6.

14. ibid., Section 10-5.

15. Holman, J.P., Heat Transfer, Section 8-16. Fourth Edition, McGraw-Hill Book Company, New York (1976).

16. ibid., Section 2-10.

17. Shampine, L.F. and Watts, H.A., "DEPAC -- Design of a User Oriented Package of ODE Solvers," Sandia National Laboratories Report SAND79-2374, Albuquerque, New Mexico (1979).

18. Baer, M.R., Benner, R.E., Gross, R.J., and Nunziato, J.W., "Modeling and Computation of Deflagration-to-Detonation Transition (DDT) in Reactive Granular Materials," Lectures in Applied Mathematics, 24, American Mathematical Society, Providence, RI, 479 (1986). 


\section{Appendix A XCHEM INPUT \& OUTPUT FILES}

\section{A.1 Ignition Experiment with Constant Temperature Boundary}

An input file for the One-Dimensional Time to Explosion (ODTX) experiments is given in this section. In the ODTX experıments, preheated alumınum anvils were used to confine $1.27 \mathrm{~cm}$ diameter spherical samples of explosive. Heaters were used to control the temperature of the anvils to $\pm 0.2 \mathrm{~K}$, and the primary measurement was the time to breach of confinement. The anvil confinement sealed the samples to pressures up to $1500 \mathrm{~atm}$. These well controlled experiments provide a simple one-dimensional geometry with constant boundary temperature conditions.

The following input file, xchem.in, also contains an example of input for a generic reactive material and nonreactive material, TATB and nichrome, respectively. The TATB reacts by a three-step mechanism. Following the input file, a partial listing of the output file $x c h e m$.out is given. This output file was obtained by running XCHEM on a CRAY.

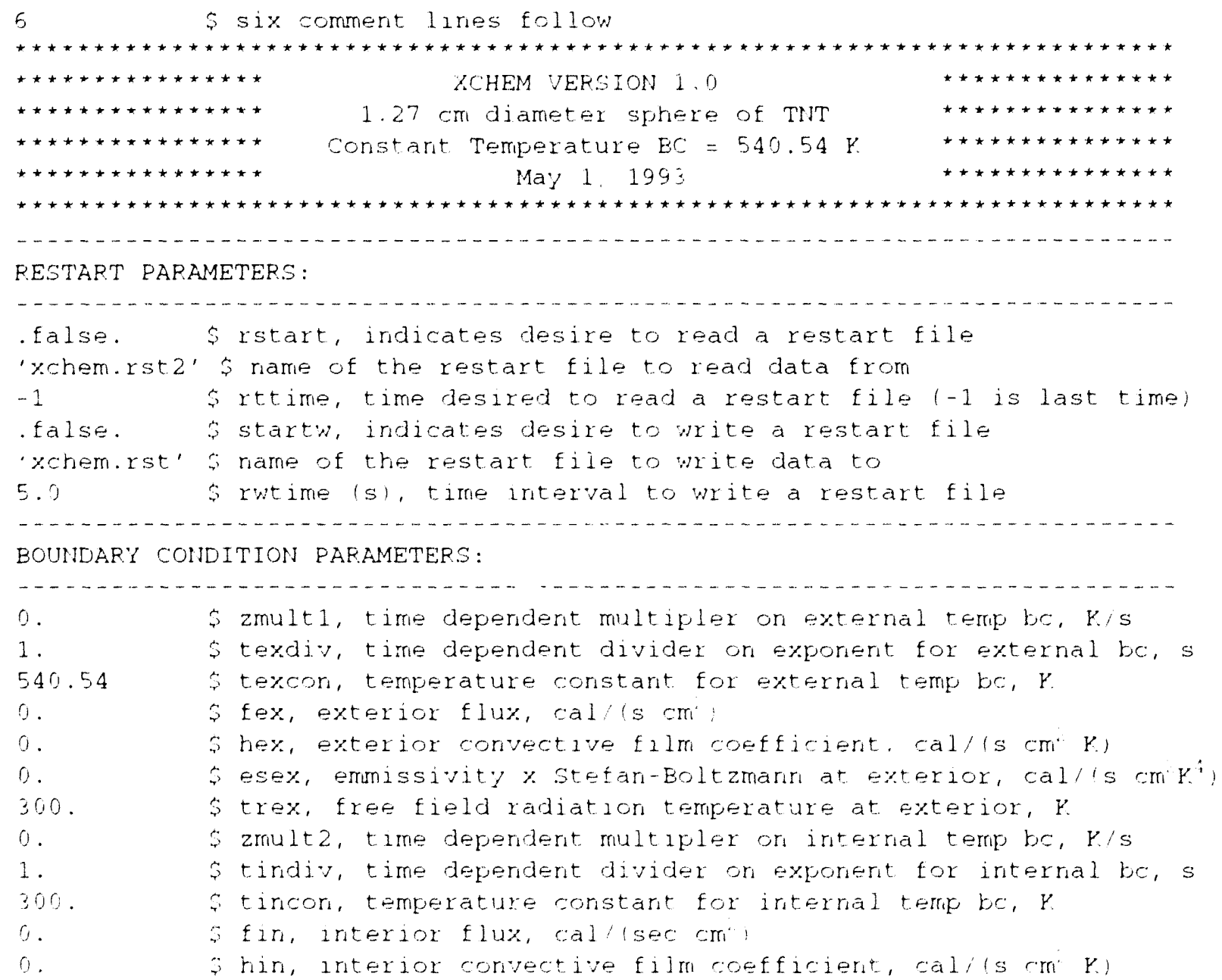




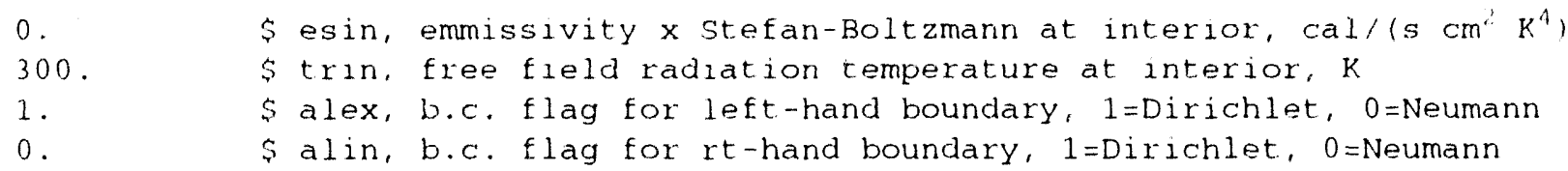

GENERAL INPUT PARAMETERS:

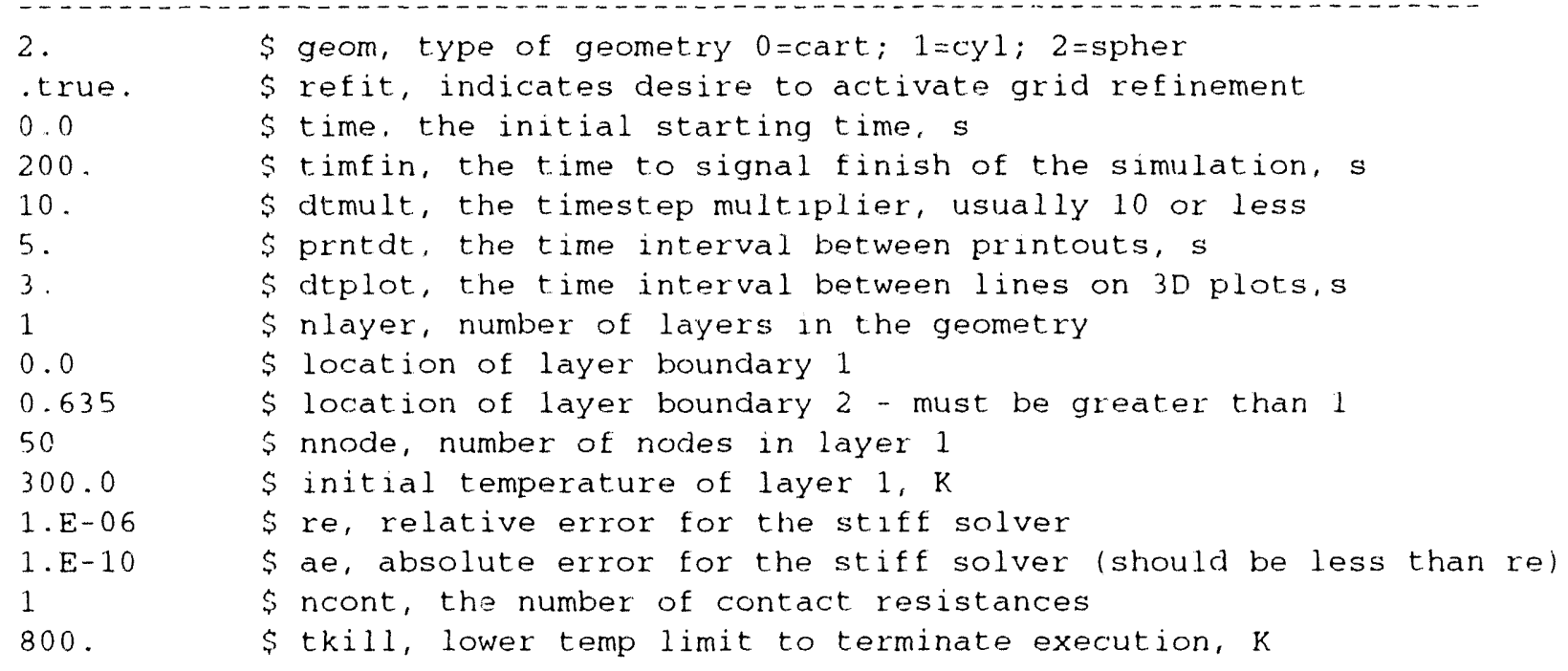

PLOTTING PARAMETERS:

true.
'plot. inp' $\$$ iplot, indicates desire for plots
'ODTX for TNT at $540.5 \mathrm{~K}^{\prime}$

MATERIAL PARAMETERS:

\begin{tabular}{|c|c|}
\hline 1 'hmx' & $\$$ material 1 is $h m x$ \\
\hline 2 'tatb' & $\$$ material 2 is tatb \\
\hline 3 ' $\mathrm{rdx}$ ' & $\$$ material 3 is $\mathrm{rdx}$ \\
\hline 4 'tnt' & $\$$ material 4 is tnt \\
\hline 5 'nc' & $\$$ material 5 is nitrocellulose \\
\hline 6 'genrt' & $\$$ material 6 is generic reactive material \\
\hline 7 'alum' & $\$$ material 7 is aluminum \\
\hline 8 ' $\mathrm{Cu}$ ' & $\$$ material 8 is copper \\
\hline 9 'be' & $\$$ material 9 is beryllium \\
\hline 10 'ti' & $\$$ material 10 is titanium \\
\hline $11^{\prime} \operatorname{ss} 304^{\prime}$ & $\$$ material 11 is 304 stainless steel \\
\hline 12 'gennrt' & $\$$ material 12 is generic nonreactive material \\
\hline 1 & \$ nentry, the following 1 entries are for volume fraction \\
\hline 141.0 & $\$$ volume fraction for layer 1 , material 4 (tnt) \\
\hline true. & $\$$ r fadd indicates desire to add sixth reactive material \\
\hline 33 & $\$$ imax, number of species; jmax, number of reactions \\
\hline 1 'solid' & $\$$ species 1 for generic rxn is a solid \\
\hline
\end{tabular}




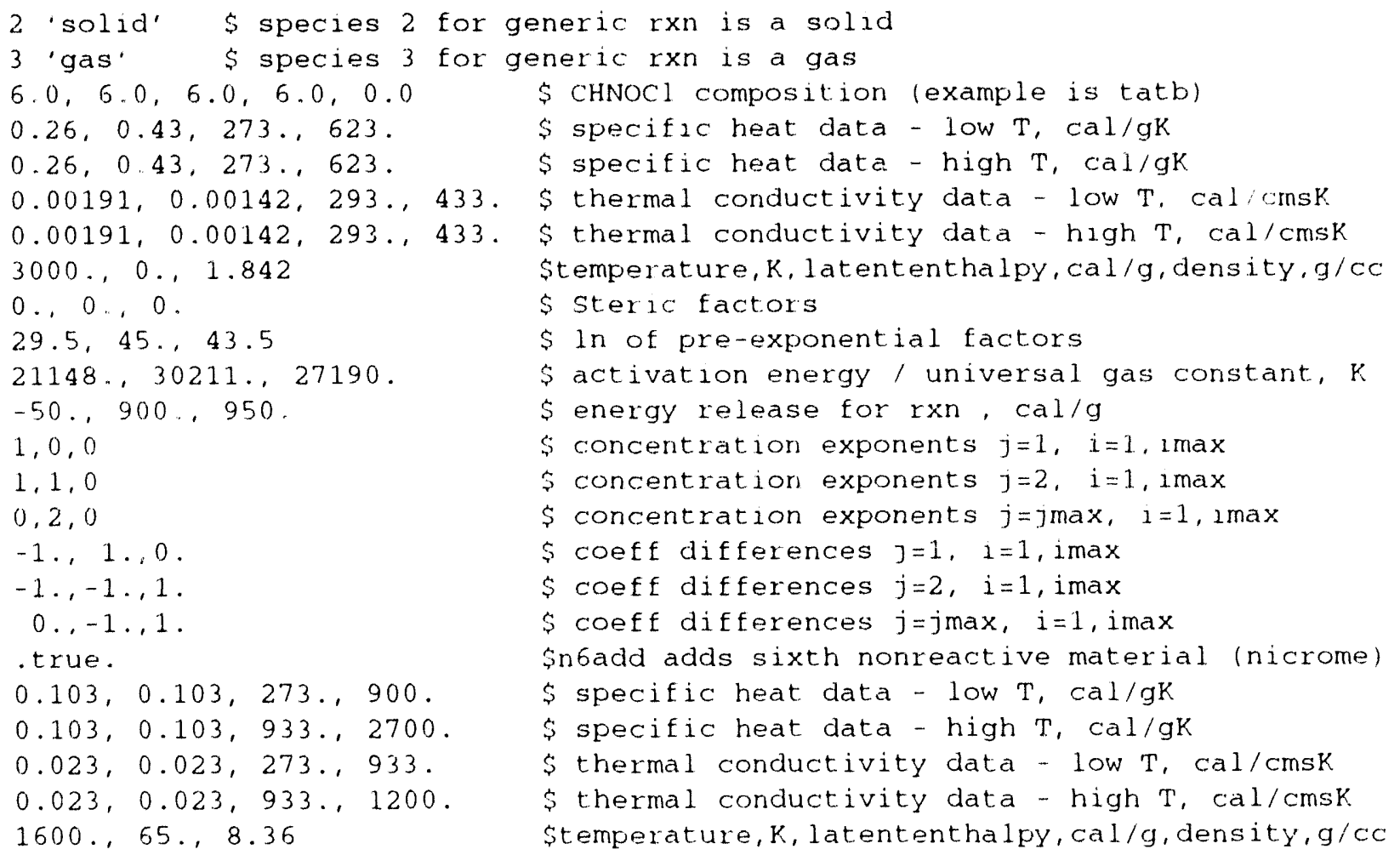

The following output file is only a partial listing:

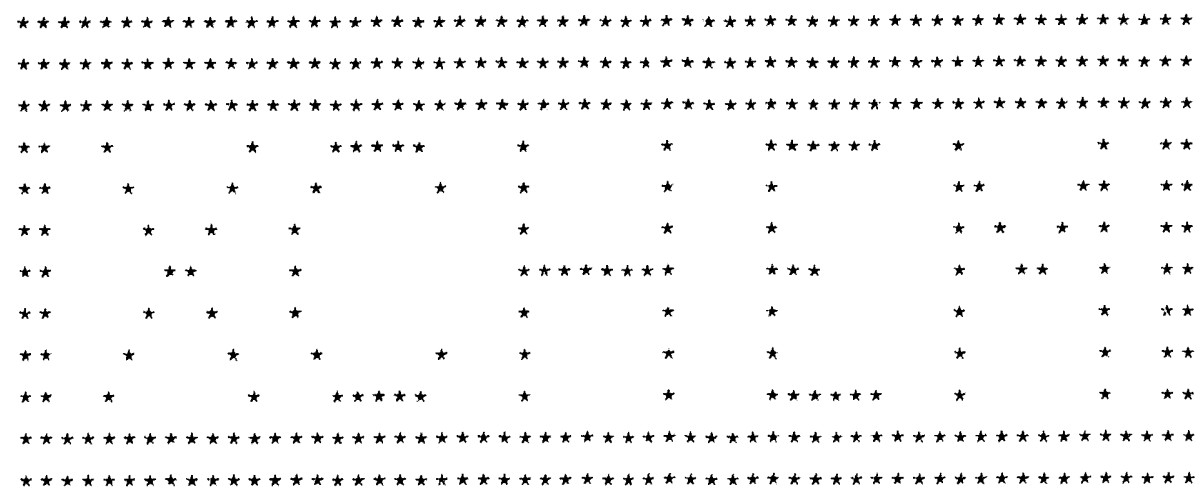

Welcome to XCHEM version 1.0

Layer 1 start at node 1 and ends at node 50 for a total of 50 nodes Low pressure viscocity (poise) $=1.5296422224694 \mathrm{E}-4$

Low pressure thermal cond (erg/cm-s-K) $=1927.968079334$

Residual gas mixture density $(\mathrm{gm} / \mathrm{cc})=0.3084485919013$

Gas viscosity high pressuxe constant $=36.92860896051$

Gas thermal cond high pressure constant $=1005.016688296$

Time $=3.446$

Time $=175.3$ 


\begin{tabular}{|c|c|c|}
\hline Distance & Temp & TNT \\
\hline $0.63500 E-02$ & 560.0 & 0.8563 \\
\hline $0.76200 \mathrm{E}-01$ & 561.3 & 0.8507 \\
\hline 0.15240 & 565.4 & 0.8321 \\
\hline 0.19050 & 568.9 & 0.8162 \\
\hline 0.22860 & 573.8 & 0.7940 \\
\hline 0.26670 & 580.8 & 0.7630 \\
\hline 0.30480 & 591.0 & 0.7183 \\
\hline 0.33338 & 602.6 & 0.6599 \\
\hline 0.35242 & 513.7 & 0.6258 \\
\hline 0.37147 & 630.1 & 0.5544 \\
\hline 0.38735 & 652.5 & 0.4870 \\
\hline 0.39687 & 675.2 & 0.4141 \\
\hline 0.40164 & 692.9 & 0.3602 \\
\hline 0.40640 & 720.2 & 0.2841 \\
\hline 0.41115 & 779.1 & 0.1485 \\
\hline 0.41315 & 848.3 & $0.4788 \mathrm{E}-01$ \\
\hline 0.41354 & 876.1 & $0.2521 E-01$ \\
\hline 0.41394 & 921.3 & $0.5545 E-02$ \\
\hline 0.41434 & 1035 & $0.1069 E-04$ \\
\hline 0.11473 & 1591. & $-0.1039 E-14$ \\
\hline 0.41513 & 1596. & $0.9839 E-24$ \\
\hline 0.41553 & 1597 . & $-0.1178 E-24$ \\
\hline 0.41593 & 1181. & $-0.4593 E-12$ \\
\hline 0.41532 & 951.9 & $0.1925 E-02$ \\
\hline 0.41672 & 892.3 & $0.1610 \mathrm{E}-01$ \\
\hline 0.41712 & 859.2 & $0.3773 E-01$ \\
\hline 0.41751 & 835.1 & $0.61 .10 \mathrm{E}-01$ \\
\hline 0.41791 & 818.0 & $0.8289 E-01$ \\
\hline 0.41831 & 803.8 & 0.1042 \\
\hline 0.41870 & 792.6 & 0.1222 \\
\hline 0.41910 & 782.7 & 0.1399 \\
\hline 0.41989 & 767.4 & 0.1597 \\
\hline 0.42069 & 754.4 & 0.1979 \\
\hline 0.42148 & 744.1 & 0.2207 \\
\hline 0.42228 & 735.4 & 0.2417 \\
\hline 0.42307 & 727.9 & 0.2597 \\
\hline 0.42386 & 721.3 & 0.2769 \\
\hline 0.42456 & 715.4 & 0.2915 \\
\hline 0.42545 & 710.1 & 0.3056 \\
\hline 0.42704 & 700.9 & 0.3295 \\
\hline 0.42863 & 593.0 & 0.3517 \\
\hline 0.43021 & 586.2 & 0.3697 \\
\hline 0.43815 & 661.6 & 0.4399 \\
\hline 0.44768 & 542.8 & 0.4938 \\
\hline $0.465,73$ & 619.0 & 0.5608 \\
\hline 0.48578 & 503.2 & 0.5027 \\
\hline 0.50483 & 591.3 & 0.6314 \\
\hline 0.53975 & 574.5 & 0.5537 \\
\hline 0.56833 & 553.4 & 0.5752 \\
\hline
\end{tabular}




$\begin{array}{lll}0.58738 & 556.6 & 0.6750 \\ 0.60643 & 550.2 & 0.6657 \\ 0.62071 & 545.4 & 0.6481 \\ 0.62230 & 544.8 & 0.6451 \\ 0.62389 & 544.3 & 0.6419 \\ 0.62548 & 543.8 & 0.6384 \\ 0.62706 & 543.2 & 0.6346 \\ 0.62865 & 542.7 & 0.6303\end{array}$

location of max temp point $=0.415528125 \mathrm{~cm}$

nondimensional location of max temp point $=0.66098484848$

Sandia National Laboratories

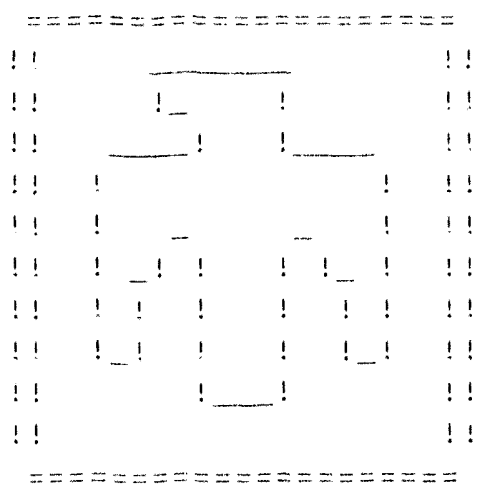

Completion of xchem

\section{A.2 Ignition Experiment with Linearly Ramped Temperature Boundary}

In the linearly ramped ODTX experiments, a $2.54 \mathrm{~cm}$ diameter cylinder of nitrocellulose ball powder was heated with a linear increase in temperature until a burst disk relieved confinement. These experiments provide a simple one-dimensional geometry with variable boundary condition.

The following input file, xchem.in, also contains an example of input for a generic reactive material and nonreactive material, respectively. The generic reactive material has constant thermal conductivity and heat capacity. The generic reactive material has a simple one-step reaction mechanism. Following the input file, a partial listing of the output file xchem.out is given.

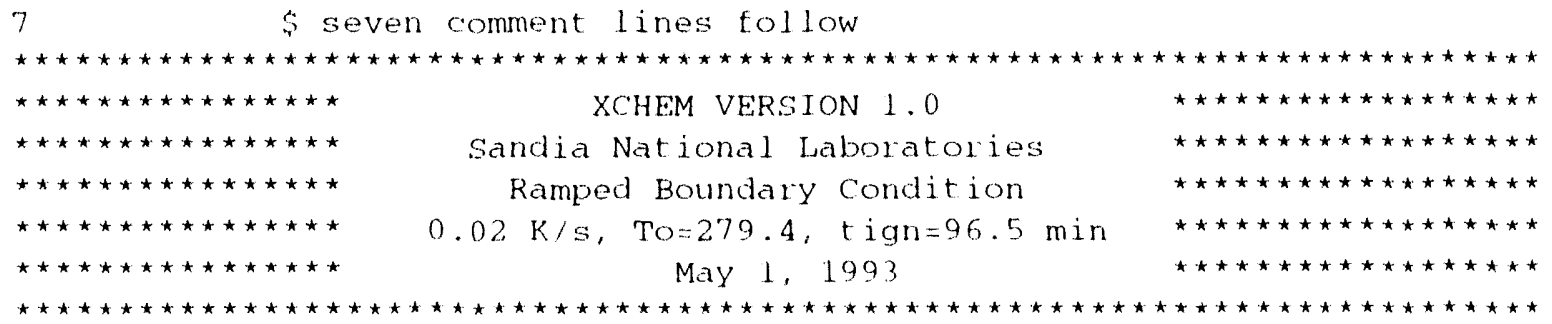

RESTART PARAMETERS: 


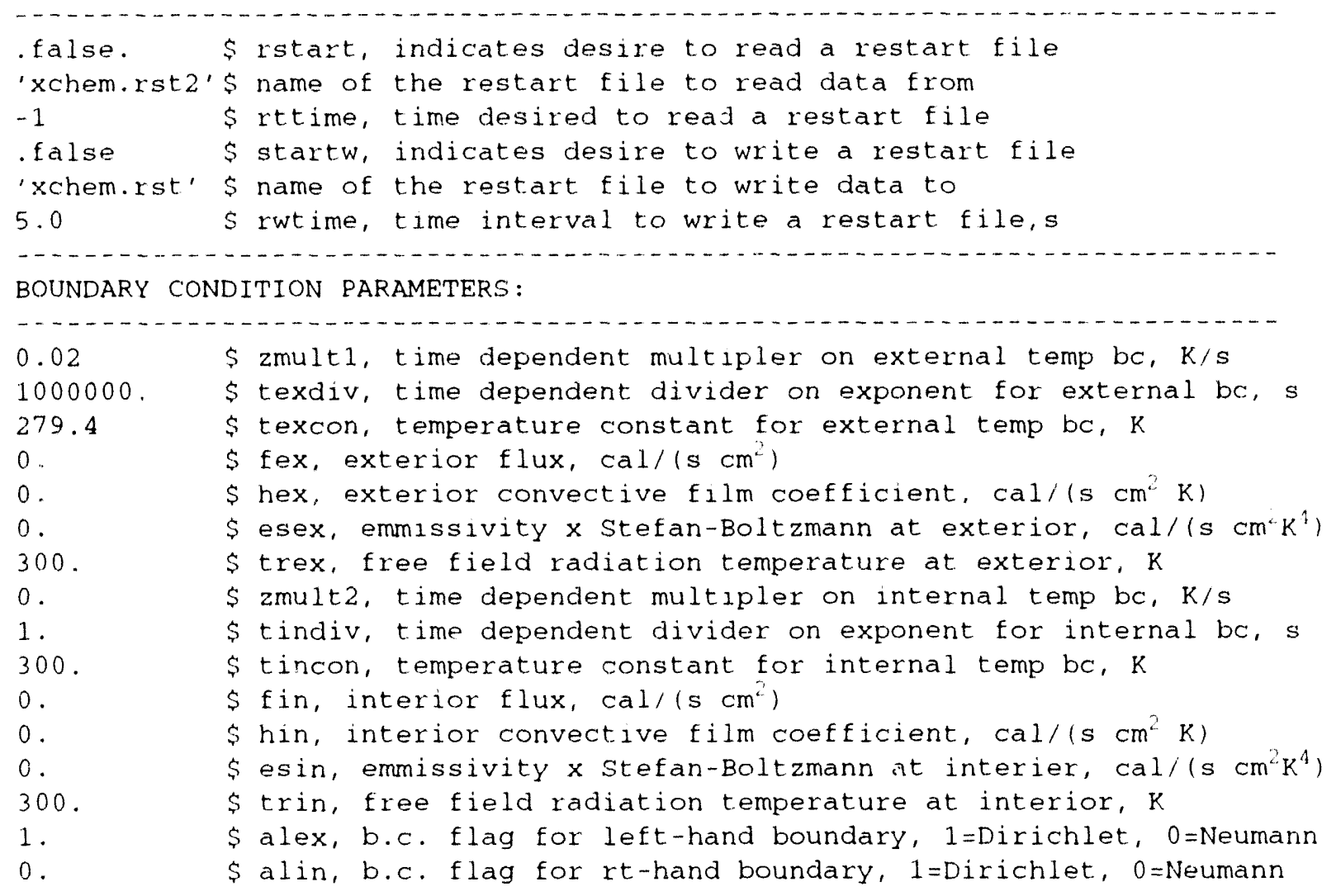

GENERAL INPUT PARAMETERS:

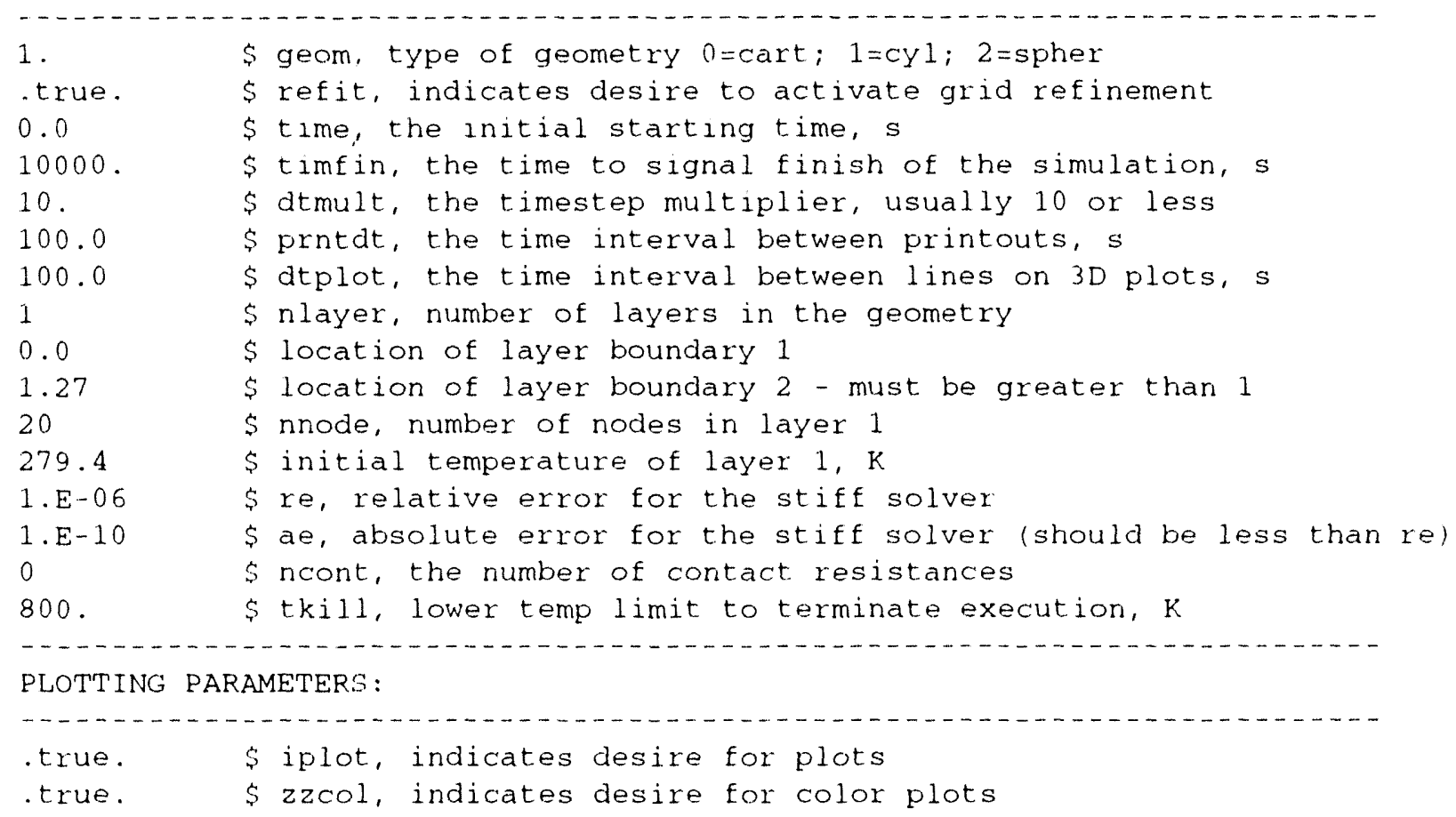


'plot.inp' \$ name of the plot rata file to be written

'ODTX for NC at $0.02 \mathrm{k} / \mathrm{s}^{\prime}$

\section{MATERIAL PARAMETERS:}

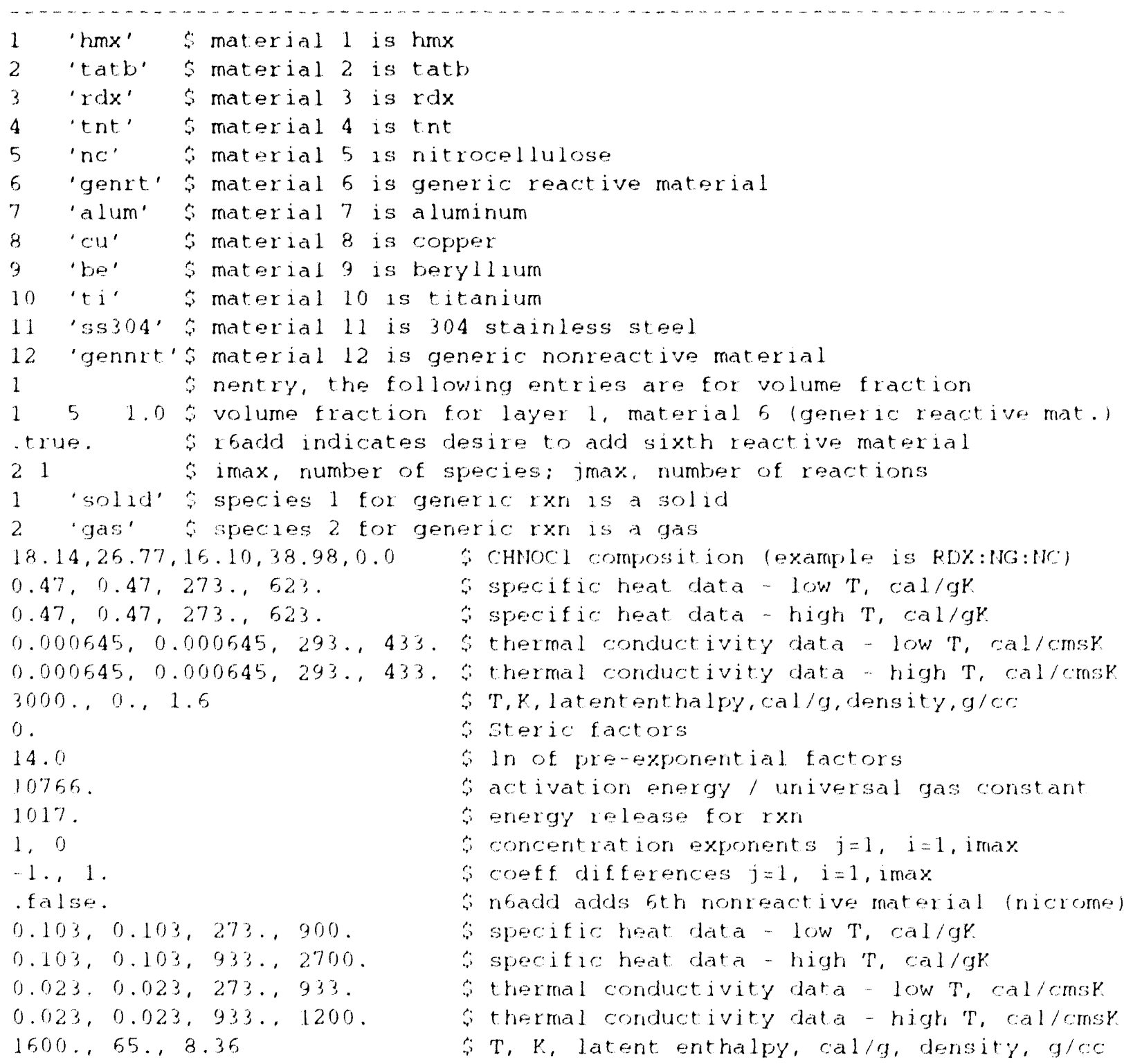

\section{The following output file is only a partial listing:}

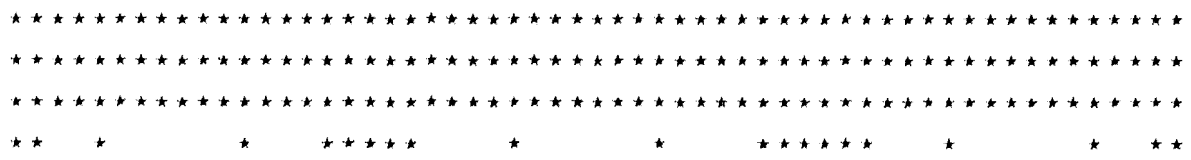




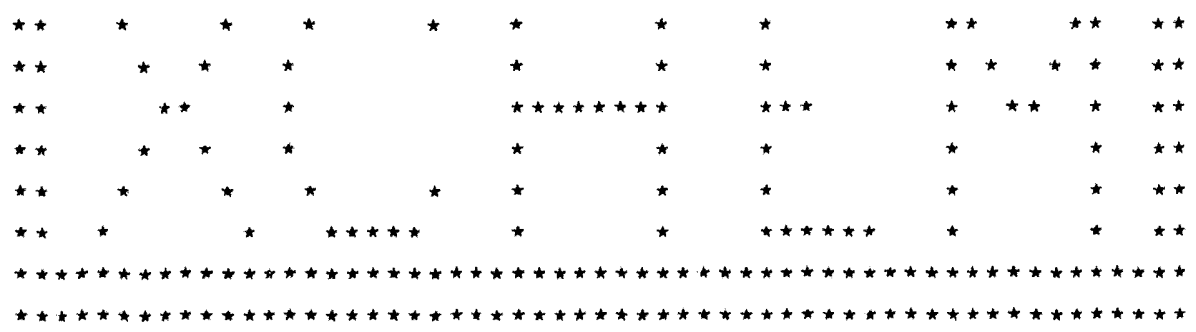

Welcome to XCHEM version 1.0

Layer 1 start at node 1 and ends at node 20 for a tota 1 of 20 nodes Low pressure viscocity (poise) $=1.5872186276507 \mathrm{E}-4$

Low pressure thermal cond $(\mathrm{erg} / \mathrm{cm}-\mathrm{s}-\mathrm{K})=1936.271672124$

Residual gas mixture density $(\mathrm{gm} / \mathrm{cc})=0.3076575218571$

Gas viscosity high pressure constant $=34.38357539012$

Gas thermal cond high pressure constant $=822.6976242246$

Time $=100.0$

Time $=6797$.

$\begin{array}{lcc}\text { Distance } & \text { Temp } & \text { NC } \\ 0.31750 \mathrm{E}-01 & 849.4 & -0.9929 \mathrm{E}-10 \\ 0.33734 \mathrm{E}-01 & 551.6 & 0.8027 \\ 0.35719 \mathrm{E}-01 & 534.3 & 0.8239 \\ 0.37703 \mathrm{E}-01 & 525.4 & 0.8351 \\ 0.3687 \mathrm{E}-01 & 519.4 & 0.8428 \\ 0 . \quad 672 \mathrm{E}-01 & 515.0 & 0.8485 \\ 0.4 \quad \mathrm{56E}-01 & 511.4 & 0.8531 \\ 0.45,41 \mathrm{E}-01 & 508.4 & 0.8570 \\ 0.476 . \mathrm{E}-01 & 505.8 & 0.8604 \\ 0.4966 \mathrm{E}-01 & 503.5 & 0.8632 \\ 0.5159 \mathrm{E}-01 & 501.5 & 0.8658 \\ 0.63500 \mathrm{E}-01 & 492.6 & 0.8776 \\ 0.87312 \mathrm{E}-01 & 481.9 & 0.8917 \\ 0.11112 & 474.9 & 0.9013 \\ 0.15081 & 466.7 & 0.9123 \\ 0.19844 & 459.7 & 0.9217 \\ 0.24606 & 454.5 & 0.9287 \\ 0.29369 & 450.3 & 0.9344 \\ 0.38100 & 444.2 & 0.9424 \\ 0.47625 & 439.0 & 0.9490 \\ 0.57150 & 434.7 & 0.9541 \\ 0.56675 & 431.1 & 0.9582 \\ 0.85725 & 425.1 & 0.9645 \\ 1.0477 & 420.0 & 0.9690 \\ 1.2382 & 415.2 & 0.9724\end{array}$

location of max temp point $=3.175 \mathrm{E}-2 \mathrm{~cm}$

nondimensional location of max temp point $=2.5641025641028 \mathrm{E}-2$

Sandia National Laboratories

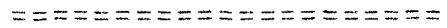

$!:$ 


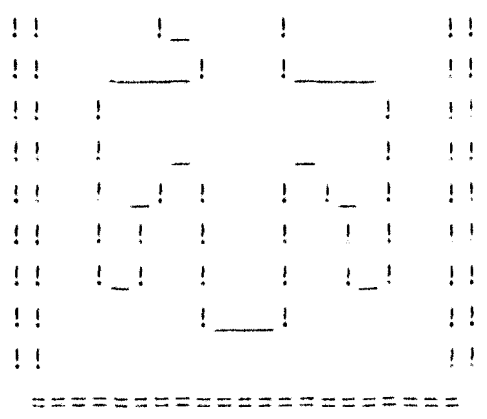

Completion of XChem

\section{A.3 Ignition Experiment with a Radiation and Convective Boundary}

In the convective and radiatively heated ODTX experiment, a $5 \mathrm{~cm}$ diameter cylinder of NC:NG:RDX propellant with a $0.64 \mathrm{~cm}$ aluminum shell was heated until thermal ignition. These experiments provide a simple one-dimensional multi-layered geometry with a convective and radiatively heated boundary condition.

The following input file, xchem.in, uses the generic reactive material with constant thermal conductivity and heat capacity to represent the propellant. The generic reactive material has a simple one-step reaction mechanism. Following the input file, a partial listing of the output file xchem.out is given.

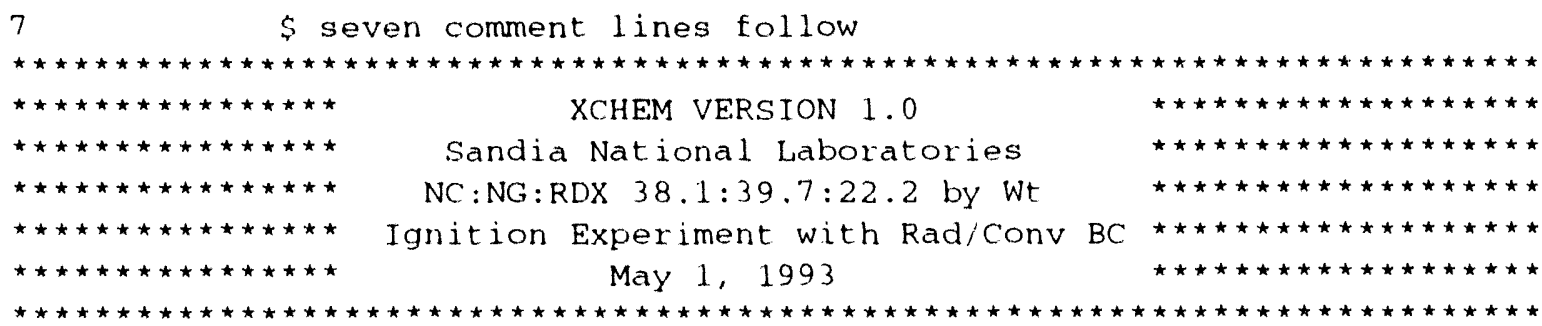

RESTART PARAMETERS :

. false. \$rstart, indicates desire to read a restart file

'xchem.rst2' \$ name of the restart file to read data from

-1 \$rtime, time desired to read a restart file

. false. $\quad \$$ startw, indicates desire to write a restart file

'xchem.rst' \$ name of the restart file to write data to

5.0 \$rwtime, time interval to write a restart file, s

BOUNDARY CONDITION PARAMETERS:

0. $\$$ zmult 1 , time dependent multipler on external temp bc, $\mathrm{K} / \mathrm{s}$

1. \$texdiv, time dependent divider on exponent for external bc, $s$ 


\begin{tabular}{|c|c|}
\hline 850 & texcon, temperature constant for external temp bc, $\mathrm{K}$ \\
\hline 0.0001356 & 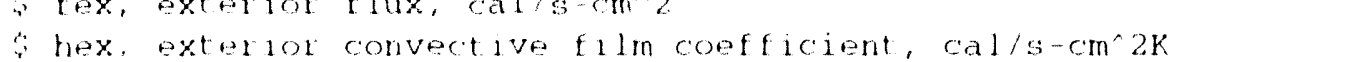 \\
\hline $2.195 \mathrm{E}-13$ & \& esex, emmissivity $x$ stefan-Boltamann at exterior, $\mathrm{cal} / \mathrm{s}_{-} \mathrm{cm}^{\wedge} 2 \mathrm{~K}^{\prime} 4$ \\
\hline 850 & $\Leftrightarrow$ trex, free field radiat lon temperature at exterior, $\mathrm{K}$ \\
\hline () & $\$$ zmult2, time dependent mult ipler on internal temp bc, $\mathrm{k} / \mathrm{s}$ \\
\hline 1 . & tindiv, time dependent divider on exponent for internal bc. s \\
\hline 300 & $\$$ tincon, tempelature constant for internal temp bc, $k$ \\
\hline 0 & \& fin, interiof flux, cal/s-cm 2 \\
\hline () & Shin, interior convective film coefficient, cal/s-cm $2 \mathrm{~K}^{\wedge} 4$ \\
\hline 1). & esin, emmissivity $x$ stetan-Boltzmann at interiot, cal/s cm $2 K^{\prime} 4$ \\
\hline 300 & S trin, free freld radiation temperature at interior. $K$ \\
\hline 0 . & $\$$ alex, b.c. flag for left-hand boundary, 1 =Dirichlet, 0 -Neumann \\
\hline 0) & Salin, b.c. flag for tt hand boundary $1=$ Darichlet, 0 -Neumann \\
\hline
\end{tabular}

GENERAL INPUT PARAMETERS:

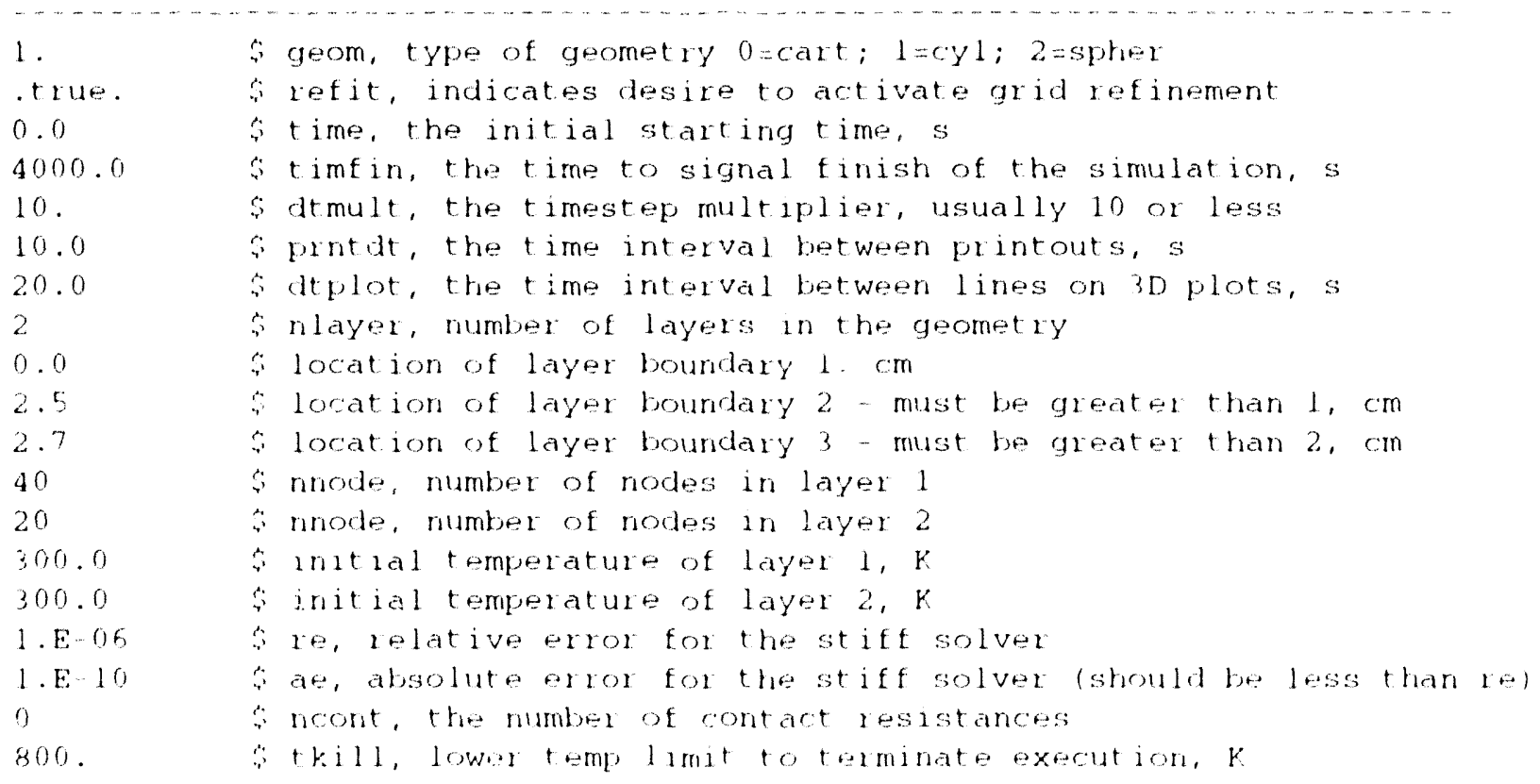

\section{PLOTTING PARAMETERS:}

the $\therefore$ iplot, imicates desir for plots

true. azeol, indicates lesile for color plots

'plot.ing' name of the plot data file to be witten

'orTy for propellant at $850 \mathrm{r}$ '

\section{MATERIAL, PARAMETERS:}

\footnotetext{
1 'hux' "mat+ilal l is hmx

2 'tatb' matelial 2 is tatb

3 'rox' matrial : is role

4 'tnt' material a 1 st $n$
} 


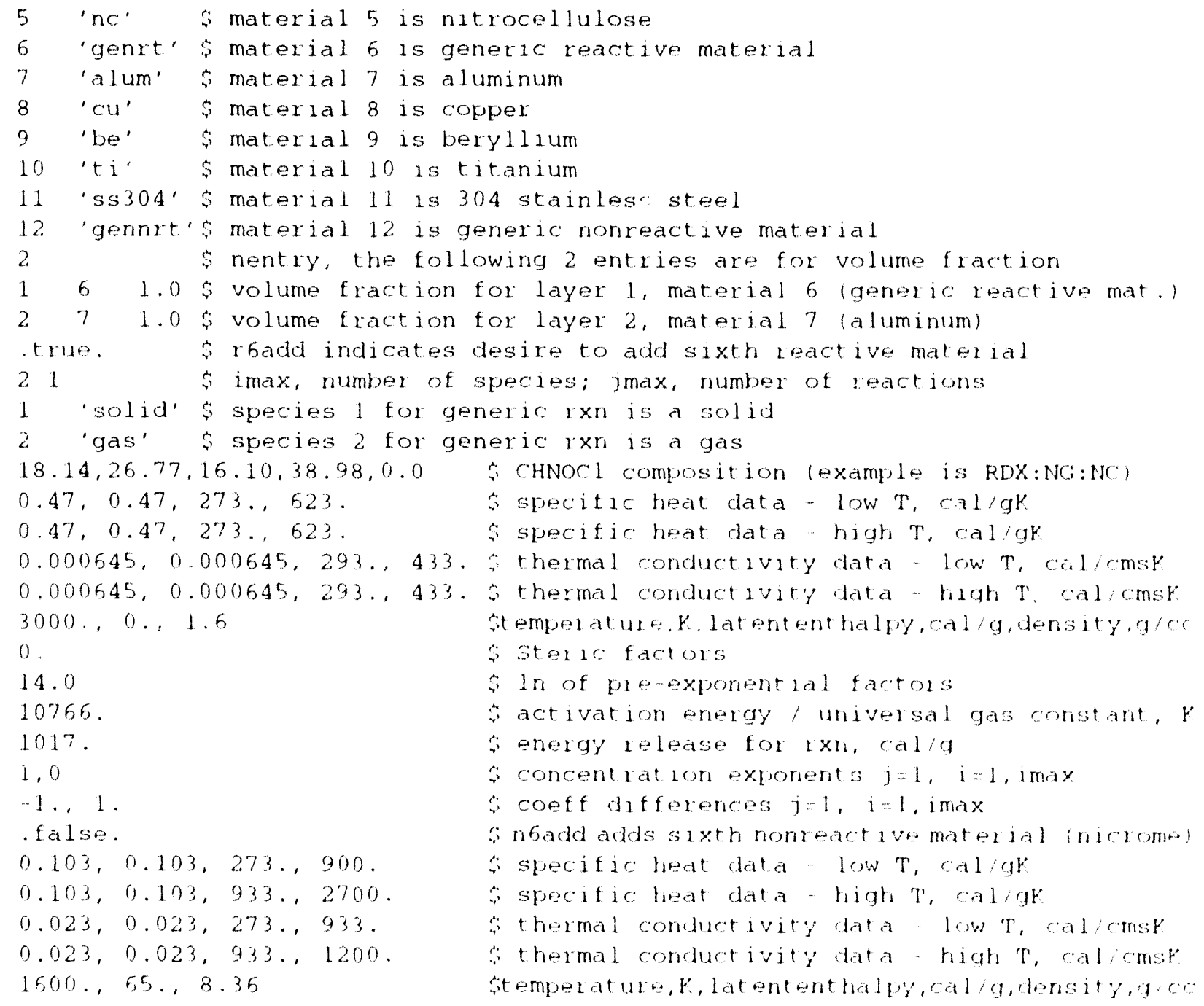

The following output file is only a partial listing:

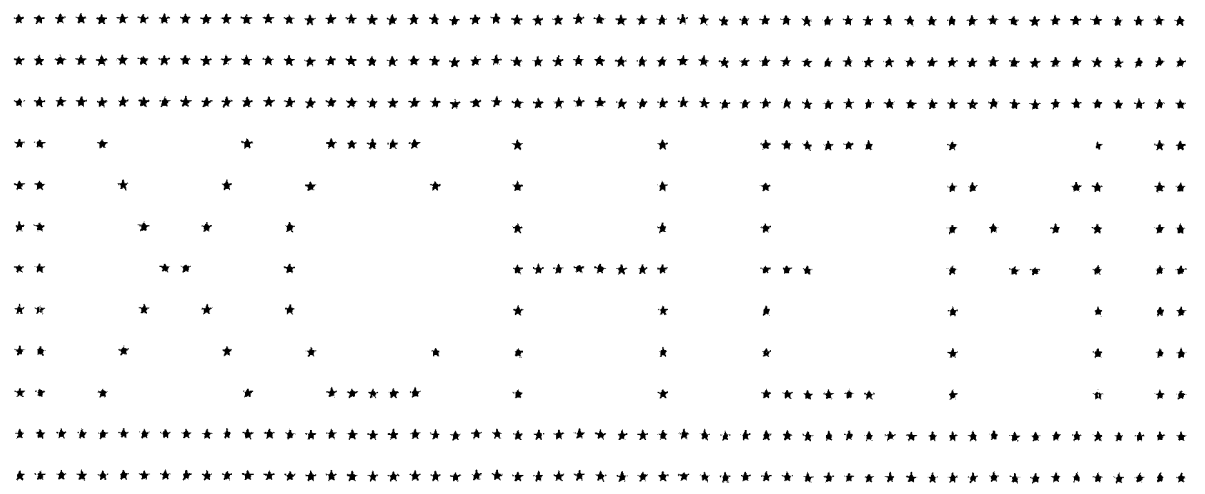

Welcome to XCHEM velsion 1.0

wryer 1 start at node 1 and ends at node $42 \mathrm{tol}$ a total of 42 romes 


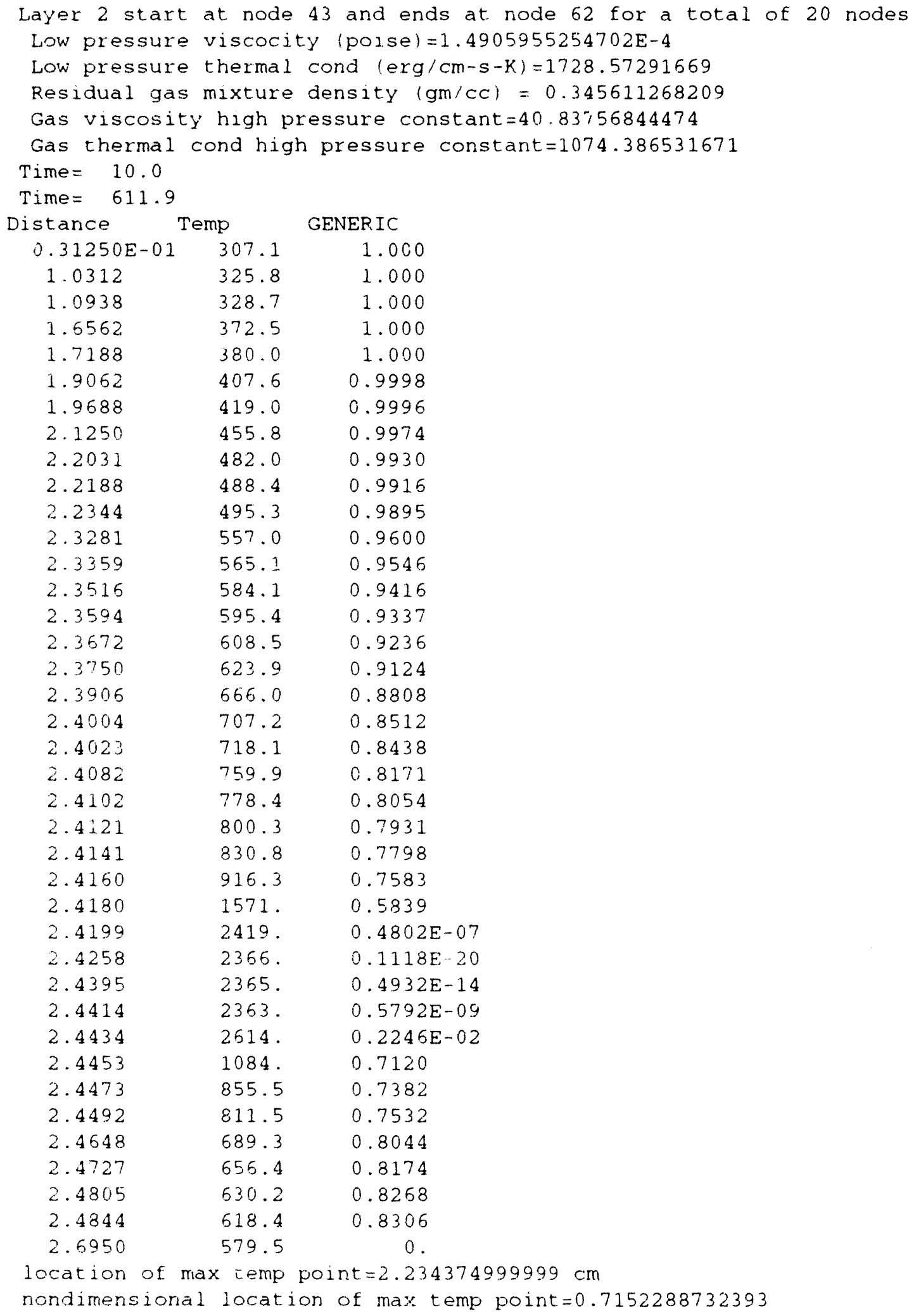




\section{Appendix B -- XPLOT Example}

Figure B.1 shows an example of the typical interactive exchange between the user and the XPLOT graphics driver. A number of questions are asked which determine scaling and choice of plots desired by the user. User responses are in bold face, while XPLOT screen responses are in regular type. The somewhat archaic " 0 " or " 1 " notation used to distinguish "yes" or "no" was used because of the varying ways in which different computing platforms handle FORTRAN character data.

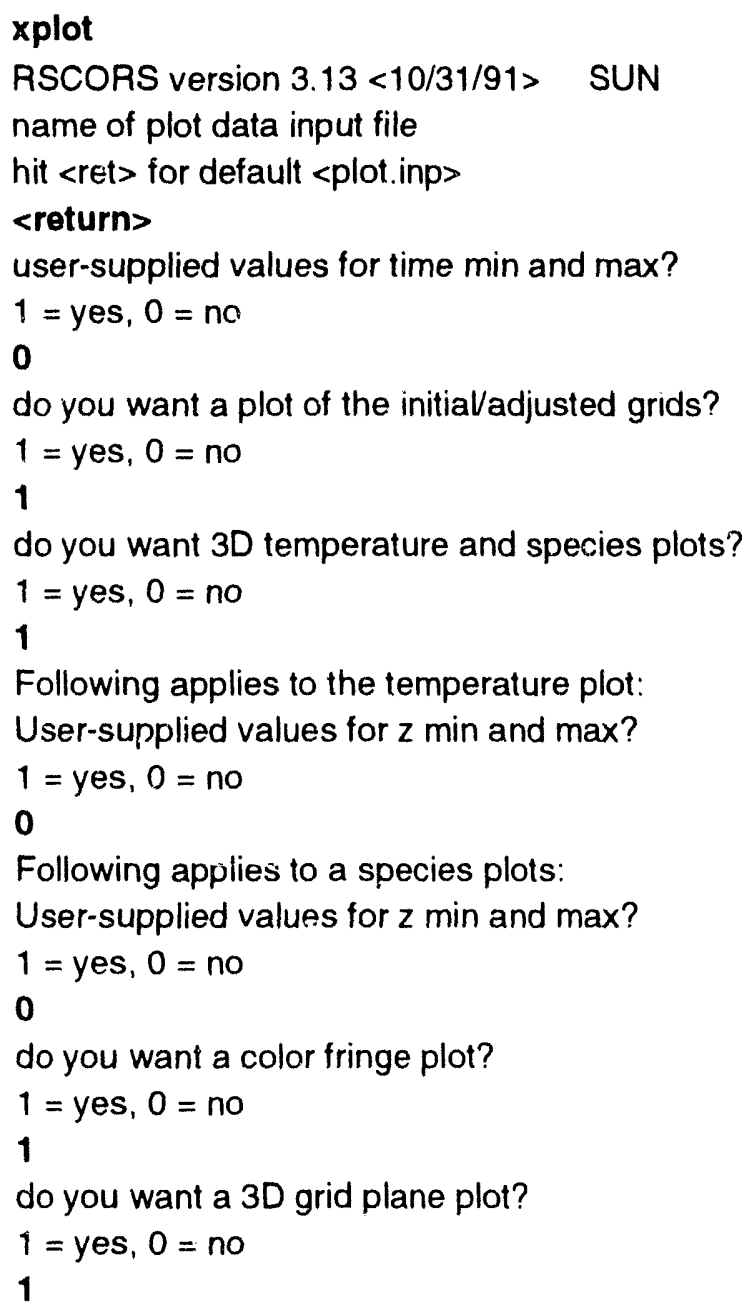

THE NUMBER OF BGPMETA FRAMES IN FILE xplot.met IS 7 THE NUMBER OF 16-BIT WORDS IN FILE xplot.met IS 401408

Figure B.1. Listing of XPLOT Screen Interaction with user

As indicated by the last two lines in Figure B.1, XPLOT has created a metafile named xplot.met. The POP software can now be used to display the data. Figure B.2 is a copy of the screen $1 / O$ interaction between POPX12 and the user. POPX12 opens an X12 window on the machine and interactively displays each plot one at a time. Again, user replies are in bold face. Note that the user in this case has specified the metafile name as xplot.met and selected 256 colors without double buffering. The desired CRT display 
format is rectangular, which has been indicated by typing " $r$ ". After this initial information, the user types " $n$ " to obtain the next plot, POP $x 12$ plots the next plot, and the plot remains on-screen until a <return> is hit, and the user hits another " $n$ " to repeat the cycle and obtain the next plot.

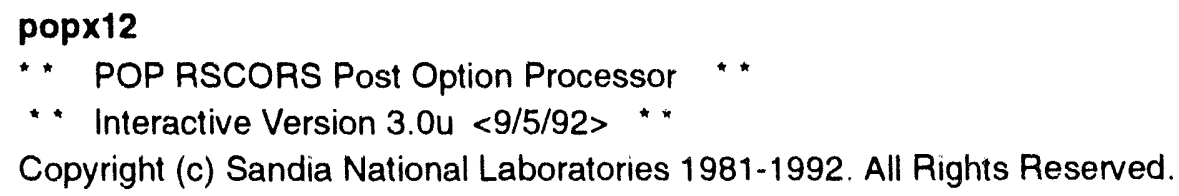

FILE ID RSCORS DATE 6/28/93 TIME 15:37:10

USER ID rigross

Select $X$ window option by number

1. 224 colors without double buffering of display

2. 256 colors without double buffering

3. 224 colors with double buffering

4. 256 colors with double buffering

5. Help

Enter option

2

This version of POP has been LINKed to device type XWIND

VDI terminal type number 779.00

Aspect ratio 0.7821 Mode interactive

Make sure you are using the correct driver.

ENTER only RETURN for HELP to questions.

ENTER HELP to next question for CRT selection help

and general RSCORS documentation.

Enter CRT display format desired :

$r$

Next frame is 1

What next :

$N$ for next frame

$F$ or $F \#$ for specific frame number \#

$S$ or $S \#$ for skip of specified number of frames \# (can be negative)

$C$ for CRT display format change

NEWFILE for new input file

EDIT for frame edit (extraction of frame to another file)

EDITFILE for edit from command file

SETWIDTH for reset of software line width for interactive CRT 
BUG for adding debug mesh overlay to next displayed frame

E for END

Next frame is 1

What next:

n

Next frame is 2

What next :

n

Next frame is 3

What next:

n

Next frame is 4

What next :

n

Next frame is 5

What next :

n

Next frame is 6

What next :

n

Next frame is 7

What next :

n

Next frame is 8

What next :

n

Next frame is 10

What next :

n

Processed 10 frames from 399360 instructions

All Done - POP

Figure B.2. Example of User Interaction with POPX12

Figure B.3 gives another POP example, this time for outputting to a black and white postscript printer. After typing "poppst", the user must again specify the name of the metafile ("xplot.met"), an option specifying color and mode of interaction with the POP software (beginners should specify one of the batch options), type of display (hit <return> for help on choices), and line width (always type " $n$ "). 


\section{poppst}

* POP RSCORS Post Option Processor * *

* * Interactive Version $3.0 \mathrm{u}<9 / 5 / 92>$ * *

Copyright (c) Sandia National Laboratories 1981-1992. All Rights Reserved.

Enter input_file_name or help

xplot.met

FILE ID RSCORS DATE 6/29/93 TIME 14:08:52

USER ID rjgross

This VDI PostScript driver has ten options.

1. black \& white, batch, no polygon fill

2. black \& white, interactive, no polygon fill

3. black \& white, batch, polygon fill

4. black \& white, interactive, polygon fill

5. color, batch

6. color, interactive

7. color, batch, black-white interchange

8. color, interactive, black-white interchange

9. color, batch, black background

10. color, interactive, black background

Enter option number

1

All Done - POP

This version of POP has been LINKed to device type PSTb\&W

VDI terminal type number 799.10

Aspect ratio 0.7500 Mode batch

Make sure you are using the correct driver.

ENTER only RETURN for HELP to questions.

ENTER HELP to next question for CRT selection help and general RSCORS documentation.

Enter CRT display format desired :

S

The current minimum line width is 0.05

Do you want to reset $\langle\mathrm{Y}$ or $\mathrm{N}\rangle$ :

n

POP output file name is popout.pst

You must queue file to device

Processed 10 frames from 399360 instructions

$\%$ Ipr -Pprinter popout.pst

Figure B.3. Example of User Interaction with POPPST 


\section{Appendix C -- Derivation of the Concentration Exponent Matrix and the "Progress Variable" Matrix}

$X C H E M$ input allows the user the capability to add another reactive material to the database and use it in normal XCHEM calculations, either alone or in combination with the materials in the standard XCHEM database. Two matrices that are part of the database of a reactive material are: (1) the matrix of concentration exponents, and (2) the coefficients on the $j$ reaction rates, which form what we term the progress variable matrix. These two matrices convey the important characteristics of the species and reactions to the chemical kinetics software that computes the right-hand side of equation (32). Since some users may wish to add different materials, or even change the reaction mechanisms of materials currently present in the database, familiarity with the procedure for determining the individual values in the two matrices is required In this section, we explain in detail the methodology used in determining the matrices for concentration exponents and the progress variables for a sequential step mechanism, used for HMX and RDX, and for an autocatalytic three-step mechanism, such as the one used for TATB and TNT.

\section{C.1 Sequential Step Mechanism (HMX and RDX)}

The sequential step mechanism, as given in Section (2.4), is:

$$
\begin{array}{lc}
\text { species equation } 1(i=1) & A \rightarrow B \\
\text { species equation } 2(i=2) & B \rightarrow 2 C \\
\text { species equation } 3(i=3) & 2 C \rightarrow D
\end{array}
$$

To determine the values of the concentration exponent matrix, we need only examine terms on the lefthand side of the above equations. Let $A$ be material 1 , let $B$ be material 2 , and $C$ be material 3 . In words, the concentration exponent value, $\mu_{4}$, is the material concentration multiplier of species reaction equation $i$ for material $\mathrm{j}$. In the first species reaction equation in the above mechanism, material 1 , or $\mathrm{A}$, has the understood multiplier 1 . Thus, on the left-hand side of species equation 1, material 1 has a concentration exponent of value "1". For species equation 1, material 2 is not present on the left-hand side, so the concentration exponent has a value of " 0 ". Likewise, material 3 in species equation 1 , which is $\mu_{13}$, also has a value of " 0 ". Thus, the first row of the concentration exponent matrix is :

$$
\mu_{1 j}=\left[\begin{array}{lll}
1 & 0 & 0
\end{array}\right]
$$

To compute the second row of the concentration exponent matrix, we focus on the second species reaction equation. Since only material B (material 2 ) is present in this second equation, we expect that only $\mu_{22}$ has a nonzero value. Thus, the second row of the concentration exponent matrix is:

$$
\mu_{2 j}=\left[\begin{array}{lll}
0 & 1 & 0
\end{array}\right]
$$

For the third row, only material $\mathrm{C}$ (material 3) is present, and thus $\mu_{33}$ will be the only nonzero term. The multiplier on material $C$ for this reaction is 2 , so that is the proper value for $\mu_{33}$ in this case. The third row, then, is:

$$
\mu_{3 j}=\left[\begin{array}{lll}
0 & 0 & 2
\end{array}\right]
$$

The entire concentration exponent matrix for this sequential step mechanism, which is the mechanism used in XCHEM for both HMX and RDX is: 


$$
\mu_{i j}=\left[\begin{array}{lll}
1 & 0 & 0 \\
0 & 1 & 0 \\
0 & 0 & 2
\end{array}\right]
$$

In order to determine the progress variable matrix, we resort to basic chemical kınetics and write the basic time-dependent change in concentration of each species as

$$
\begin{gathered}
\frac{d N_{A}}{d t}=-k_{1} N_{A} \\
\frac{d N_{B}}{d t}=k_{1} N_{A}-k_{2} N_{B}
\end{gathered}
$$

$$
\frac{d N_{C}}{d t}=k_{2} N_{B}-k_{3} N_{C} N_{C}
$$

where $N_{1}$ (dimensionless in XCHEM) tracks the creation or destruction of species $i$, and $k_{i}$ are the kinetic coefficients. Using Equation (4), the above system is reduced to:

$$
\begin{gathered}
\frac{d N_{A}}{d t}=-r_{1} \\
\frac{d N_{B}}{d t}=r_{1}-r_{2} \\
\frac{d N_{C}}{d t}=r_{2}-r_{3}
\end{gathered}
$$

where the $r_{j}$ 's are the reaction rates. The progress variable matrix is obtained by taking the coefficients on the above equation system, so that:

$$
v_{i j}=\left[\begin{array}{ccc}
-1 & 0 & 0 \\
1 & -1 & 0 \\
0 & 1 & -1
\end{array}\right]
$$

\section{C.2 Auto-catalytic Three-Step Mechanism (TATB and TNT)}

The auto-catalytic three-step mechanism, as given in Section (2.4), is: 


$$
\begin{array}{cc}
\text { species equation } 1(i=1) & A \rightarrow B \\
\text { species equation 2 }(i=2) & A+B \rightarrow C \\
\text { species equation } 3(i=3) & 2 B \rightarrow C
\end{array}
$$

Again, we need the concentration exponent matrix. $\mu_{11}$, and the progress variable matrix, $v_{1}$. In determining the concentration exponent matrix, we may note that the first species equation is identical to that of the sequential step mechanism. Thus, the first row of the concentration exponent matrix for this mechanism is identical to the first row determined from the sequential step mechanism:

$$
\mu_{1 /}=\left[\begin{array}{lll}
1 & 0 & 0
\end{array}\right]
$$

The second species equation contains both $A$ and $B$ on the left-hand side, each with a coefficient of " 1 ", so that the second row is composed as:

$$
\mu_{21}=\left[\begin{array}{lll}
1 & 1 & 0
\end{array}\right]
$$

Finally, the third species equations only contains $B$ on the left-hand side with a coefficient of "2", so that the third row is composed as:

$$
\mu_{3 /}=\left[\begin{array}{lll}
0 & 2 & 0
\end{array}\right]
$$

so that the entire concentration exponent matrix for the autocatalytic three-step mechanism is:

$$
\mu_{i j}=\left[\begin{array}{lll}
1 & 0 & 0 \\
1 & 1 & 0 \\
0 & 2 & 0
\end{array}\right]
$$

Again, to determine the progress variable matrix, we write the basic change in concentration of each of the three species as:

$$
\begin{gathered}
\frac{d N_{A}}{d t}=-k_{1} N_{A}-k_{2} N_{A} N_{B}=-r_{1}-r_{2} \\
\frac{d N_{B}}{d t}=k_{1} N_{A}-k_{2} N_{A} N_{B}-k_{3} N_{B} N_{B}=r_{1}-r_{2}-r_{3} \\
\frac{d N_{C}}{d t}=k_{2} N_{A} N_{B}+k_{3} N_{B} N_{B}=r_{2}+r_{3}
\end{gathered}
$$

where the $r_{j}$ 's are the reaction rates. The progress variable matrix is obtained by taking the coefficients on the above equation system, so that: 


\section{Distribution}

\section{Brigham Young University}

Attn: Beckstead, M.

Department of Chornical Engineering

Provo, UT 84602

\section{Brigham Young University}

Attn: Radulovic, P. T.

Department of Mechanicall Engineering

Provo, UT 84602

Lawrence Livermore National Laboratory (9)

Attn:

Nichols, A.

Tarver, C.

Westerberg, $\mathrm{K}$.

Tao, W.

Foltz, M.

Green, $L$.

Kury, J.

Simpson, R.

Souers, $P$.

P. O. Box 808

Livermore, CA 94550

Los Alamos National Laboratory (7)

Attn:

Sheffield, S.

Asay, B.

Jaeger, D.

Dienes, J.

Howe, $P$.

Storm, C.

Kennedy, J.

P. O. Box 1663

Los Alamos, NM 87545

Naval Alr Warfare Center (9)

Attn: Boggs, T. L.

Price, C. F.

Covino, J.

Dimaranan, $\mathrm{L}$.

Atwood, A. I.

Chan, M. L.

Lundstrom, E. A.

Richter, H. P.

Heimdahl, R.

China Lake, CA 93555-6001
Naval Surface Warfare Center (3)

White Oak Laboratory

Attn: Kim, K.

Bernecker, R. R.

Sandusky, H. W.

Silver Springs, MD 20910

North Carolina State University

Attn: Horie, Y.

Department of Mechanical \&

Aeronautical Engineering

Raleigh, NC 27607

Callfornia Institue of Technology

Attn: Shepherd, J. E.

Graduate Aeronautical Laboratories

Mail Stop 105-50

Pasadena, CA 91125

Stanford Research Institute (SRI) International

Attn: $\quad$ Cowperthwaite, $M$.

333 Ravenswood Avenue

Menlo Park, CA 94025

University of IIIInols

Attn: Krier, $\mathrm{H}$.

Department of Mechanical Engineering

1206 W. Green Street

Champaign, IL 61801

University of Illinols

Attn: $\quad$ Stewart, D. S.

104 S. Wright Street

Unbana, IL 61801

University of lowa

Attn: $\quad$ Butler, P. B.

Department of Mechanical Engineering

2208 Engineering Bldg.

lowa City, IA 52245

University of Notre Dame

Attn: $\quad$ Powers, J.

Department of Mechanical Engineering \&

Aerospace

Notre Dame, IN 46556

U. S. Army Ballistic Research Laboratory (2)

Attn: Kooker, D. E.

Starkenberg, J.

Aberdeen Proving Grounds, MD 21005 
IIT Research Institute (2)

Attn: $\quad$ Austing, J. L. Tulis, A. J.

10 West 35th Street

Chicago, IL 60616-3799

U. S. Army Armament Research

Attn: Baker, E.

Development and Engineering Center

Picatinny Arsenal, NJ 07806.5000

DQA

Attn: Baudin, G.

Gramat, 46500

FRANCE

Thlokol Corporation

Attn: $\quad$ Braithwaite, P. C.

P. O. Box 707, MS 244

Brigham City, UT 84302.0707

University of Delaware

Attn: Brill, T. B.

Newark, DE 19716

NIMIC

Attn:

Delourneaux, $M$.

Brussels, 1110

BELGIUM

Enig Associates, Inc.

Attn: $\quad$ Enig, J.W.

Suite 500

11120 New Hampshire Avenue

Silver Spring MD 20904-2633

Army Research Laboratory

Attn:

Frey, R. B.

Aberdeen Proving Ground, MD

21005-5066

New Mexico Institute of Mining Tech. (4)

Attn:

Libersky, L.

Olson, D. B.

Oxley, J. C.

Persson, $P$.

Campus Station

Socorro, NM 87801

Victor Technology

Attn: $\quad$ Victor, A. C.

712 North Peg Street

Ridgecrest, CA 93555
DOE Albuquerque Operations Offlce

Acting Director, Weapons Program Division

Albuquerque, NM 87185

Battelle Memorlal Instltute (2)

Attn: Doug Everhart

Dale Trott

505 King Avenue

Columbus, $\mathrm{OH} 43201-2693$

University of Washington

Attn: Dave McTigue

Department of Geological Sciences

AJ-20

Seattle, WA 98195

Hercules Aerospace Company

Attn: $\quad$ A. Garn Butcher

Mail Stop ATR-2

Bacchus Works

Magna, UT 84044-0098

Martin Marietta Energy Systems, Inc.

Attn: Donald E. Wolch

P. O. Box 2003

Oak Ridge, TN 37831-7294 


\section{Internal Distribution}

MS0631 12300 Schwoebel, R. L.

MS0491 12333 Demmie, P. N.

MSO491 12333 D'Antonio, P.E.

MSO491 12333 Smith, R. E.

MS0345 1153 Graham, R. A.

MS1111 1421 Shadid, J. N.

MS1109 1424 Benner, R. E.

MSO820 1432 Kipp, M. E.

MSO820 1432 Yarrington, $P$.

MS0841 1500 McCloskey, D. J.

MS0836 1501 Peterson, C. W.

MS0827 1511 Gartling, D. K.

MS0827 1511 Martinez, M. J.

MS0827 1511 Rao, R. R.

MS0834 1512 Baer, M. R.

MS0834 1512 Erickson, K. L.

MSO834 1512 Gross, R. J.

MS0834 1512 Hobbs, M. L.

MS0834 1512 Ratzel, A. C.

MS0834 1512 Trott, W. M.

MS0835 1513 Moya, J. L.

MS0835 1513 Skocypec, R. D.

MSO826 1553 Blackwell, B. F.

MSO826 1553 Hermina, W., L.

MSO443 1561 Stone, C. M.

MS0437 1562 Sjaardema, G. D.

MSO437 1562 Thomas, R. K.

MSO329 2512 Harlan, J. G.

MS0326 2513 Fischer, S. H.

MSO326 2513 Mitchell, D. E.

MSO327 2514 Bonzon, L. L.

MS0327 2514 Renlund, A. M.

MS0445 5166 Setchell, R. E.

MSO457 5600 Hayes, D. B.

MS0467 5091 Nokes, K. D.

MSO405 6411 Jones, T.

MSO405 6411 Maloney, K. J.

MS0745 6418 Thompson, S. L.

MS0899 7141 Technical Library Processes

(5)

MSO619 7151 Technical Publications

MSO1119 7613-2 Doc. Processing for DOEOSTI (10)

MS9163 8302 Bauer, W.

MS9051 8351 Margolis, S.

MS9055 8353 Melius, C. F.

MS9018 8523-2 Central Technical Files

\&US GOVERNMENT PRINTING OFFICE $1993 \quad 573 \quad 110 / 80275$
(10)
MSP055 8353 Behrens, R.

MS9053 8366 Hartwig, C.

MS9043 8745 Griffiths, S. K. MS9043 $8745 \mathrm{Ke \theta}, \mathrm{R} . \mathrm{J}$.

MS1156 9333 Cooper, P. W.

MS0763 9614 Graeber, E. J.

MS0763 9614 Hartman, W. F.

MS0763 9614 Larsen, M. 

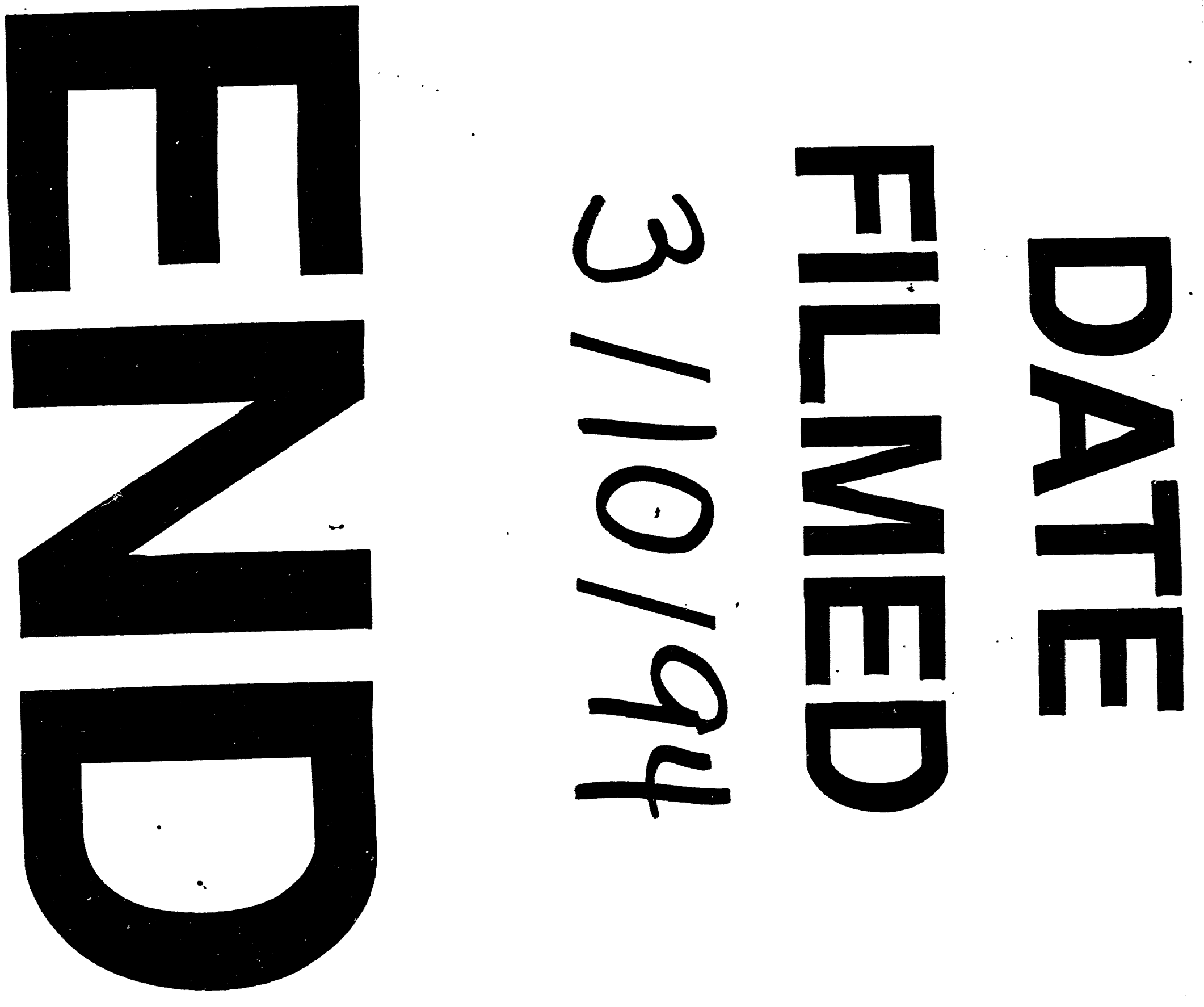


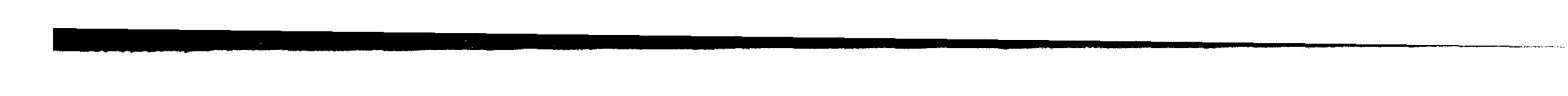

